\title{
Metal-organic framework growth at functional interfaces: thin films and composites for diverse applications
}

\author{
Darren Bradshaw*a, Ashesh Garai ${ }^{\mathrm{a}}$ and Jia Huo ${ }^{\mathrm{a}}$ \\ Received (in $X X X, X X X) X$ th $X X X X X X X X X 20 X X$, Accepted $X$ th $X X X X X X X X X 20 X X$ \\ ${ }_{5}$ DOI: 10.1039/b000000x
}

Porous metal-organic frameworks (MOFs) are highly ordered crystalline materials prepared by the selfassembly of metal ions with organic linkers to yield low density network structures of diverse topology. MOFs have attracted considerable attention over the last decade due to their facile preparation, tunable pore metrics and the ease of functionalisation of their internal surfaces, such that designer frameworks

10 with exceptional properties for application in gas-storage, separation of small molecules, heterogeneous catalysis and drug delivery are becoming commonplace. For any material to find practical utility however there is a need for processing and formulation into application-specific configurations. One way to do this is to prepare composite materials where the MOF is supported on a planar substrate or some other shaped body through interaction with functional groups at the support interface. This is a rapidly developing

15 research area, and this review provides an overview of the diverse MOF composite materials prepared up to now, organised by interface type. The importance of the interface is explored within each section and while the overall emphasis is on applications of the composites, coatings and MOF-based devices, the most widely-used and successful synthetic strategies for composite formation are also presented. (183 references)

\section{${ }_{20}$ Introduction}

Metal-organic frameworks (MOFs), sometimes known as porous coordination polymers (PCPs), are crystalline microporous materials prepared using the well-established principles of coordination chemistry. ${ }^{1-3}$ Through self-assembly of (usually)

25 transition metal ions or higher nuclearity metal clusters - often referred to as secondary building units or SBUs - with multitopic organic ligands, reticular structures with tunable pore sizes (IRMOFs) of the order of molecular dimensions are accessible. ${ }^{4}$ Framework functionality beyond their accessible porosity can 30 arise from the metal components (e.g. magnetism, catalysis), organic linkers (e.g. luminescence, chirality) or a combination of both, ${ }^{5}$ and may be pre-programmed into the framework building blocks ${ }^{6}$ or added/activated after assembly using a post synthetic modification (PSM) strategy. ${ }^{7}$ Further, the inorganic-organic

35 hybrid nature of MOFs endows the frameworks with an inherent flexibility ${ }^{8}$ leading to non-classical adsorption behaviours ${ }^{9}$, guestresponsive uptake ${ }^{10}$ and potential for significant structural rearrangement, for example through 'breathing' effects. ${ }^{11}$

As a direct result of this structural and functional diversity, 40 degree of rational design at the molecular level and relative ease of preparation, interest and research in MOFs has followed an upward exponential trajectory over the last $10-15$ years. ${ }^{5}$ The vast majority of studies this far have involved the synthesis, characterisation and to some extent optimisation of new ${ }_{45}$ functional materials for wide-ranging applications in (but not limited to) sequestration and storage of fuel and environmental gases $^{12,13}$, heterogeneous catalysis ${ }^{14}$, molecular separation (e.g. gases $^{15}$, enantiomers ${ }^{16}$ ), drug delivery ${ }^{17}$, biomedical imaging ${ }^{18}$ and sensing. ${ }^{19}$

50 There is an ever increasing awareness however, that the insoluble micron-sized crystals typically obtained from traditional MOF synthesis reactions (e.g. solvothermal methods) are not necessarily the best configuration for the applications outlined above. Consequently there are now a growing number of reports 55 of methods to prepare MOF thin films or coatings, nanoparticles (nps) and composites, which have been recently reviewed. ${ }^{20,21}$ The aim of these studies is to take MOFs beyond interesting crystals full of promise on the laboratory bench-top, and prepare or process these (directly or otherwise) into integrated devices 60 and other application-specific configurations required for commercialisation and by the future end-users of the product. This is a necessary step for MOFs, marking the next phase of their development and moving the research field into its adolescence.

65 There are a number of strategies for preparing MOF nps ${ }^{22}$ and capsules $^{23}$ primarily for biomedical applications. For other more industrially relevant applications such as separation and catalysis, MOF-based composite systems are a desirable synthesis target, of which there are four general types: (1) MOFs grown/deposited 70 onto 2- and 3-dimensional substrates using both 'top-down' and 'bottom-up' strategies ${ }^{24,25}$; (2) MOFs acting as host matrices for nanoscale objects of diverse composition ${ }^{26}$; (3) composites formed in a one-pot synthesis rather than being deposited onto a 
pre-formed support ${ }^{27}$; and (4) MOF core/shell structures, where MOF crystals are coated in protective shells, for example to increase their biocompatibility. ${ }^{28}$ To this can be added a newly emerging class, where the second component of the composite is 5 an integral part of the MOF itself. $^{29}$

The focus of this review is largely those systems in category (1), where the starting point for preparation is an interface bearing suitable functional groups - usually the same metalbinding groups expressed by the framework ligand components 10 for interaction with the surfaces of MOF crystallites. ${ }^{30}$ The resulting composite materials range from supported oriented defect-free thin films and membranes ${ }^{21}$ to shaped 3-dimensional bodies of hierarchical porosity. ${ }^{31}$ While MOF thin films have been the subject of several reviews in recent years ${ }^{20,24,32}$, the 15 purpose here is for the first time to group together all reported composites according to interface type thus providing a broad overview of the diversity of materials and application-specific configurations that have this far been prepared as this branch of MOF chemistry rapidly develops.

\section{${ }_{20}$ MOFs at metal/SAM interfaces}

Self-assembled monolayers (SAMs) are functional organic thin films that can be supported on a wide variety of substrates (e.g. metals, oxides) and, under carefully controlled synthesis conditions, can be highly ordered resembling a 2-D crystalline 25 interface. Their relative ease of preparation, spontaneous assembly, diverse functionality and ability to be patterned using facile micro-contact printing methods, make SAMs an attractive interface for MOF growth. These properties are exemplified by SAMs on Au substrates, which were the first functional surfaces 30 to be employed for the growth of MOF thin films. ${ }^{33}$ (Fig. 1)

The synthetic procedures developed as part of these pioneering studies in the assembly of MOF films are widely used for the preparation of most MOF composite materials, and due to their importance to the development of the field a discussion of the 35 two most common methods employed will be presented here. For a discussion of the wider synthesis techniques used to prepare MOF composites (including microwave-assisted ${ }^{34}$, electrochemical $^{35}$ and colloidal methods ${ }^{36}$ ) and their relative merits, the recent critical review by Shekhah et al provides an 40 excellent appraisal. ${ }^{24}$

Addition of Au-supported SAMs to a standard solvothermal MOF synthesis is not a practical method to prepare MOF thin films (or composites) given the relative fragility of the SAM/Au interface at the elevated temperatures typically employed. In 45 order to circumvent this difficulty, a number of alternative synthesis strategies have been developed for the preparation of MOF films on SAMs; methods which have been extended to prepare diverse MOF composite materials. Two of the most successful techniques are based on immersion of SAMs into aged 50 precursor solutions containing both the metal and ligand components $^{33,37}$ or by employing a liquid-epitaxial layer-by-layer (LbL) approach to MOF growth. ${ }^{38}$

MOF precursor solutions have been employed to prepare laterally patterned MOF-5 $\left(\left[\mathrm{Zn}_{4} \mathrm{O}(\mathrm{bdc})_{3}\right]\right.$ where bdc $=1,4$ 55 benzenedicarboxylate) (Fig. 1), and oriented HKUST-1 $\left(\left[\mathrm{Cu}_{3}(\mathrm{btc})_{2}\left(\mathrm{H}_{2} \mathrm{O}\right)_{3}\right]\right.$ where btc $=1,3,5$-benzenetricarboxylate $)$ thin films (Fig. 2) on functionalised SAMs on Au substrates. ${ }^{33,} 37$ The
MOF precursor solutions are prepared by dissolution of the metal ions and ligands into the bulk reaction solvent and held at 60 elevated temperature to initiate the formation of MOF nuclei; the solutions are subsequently cooled, and filtered if required, prior to SAM immersion.
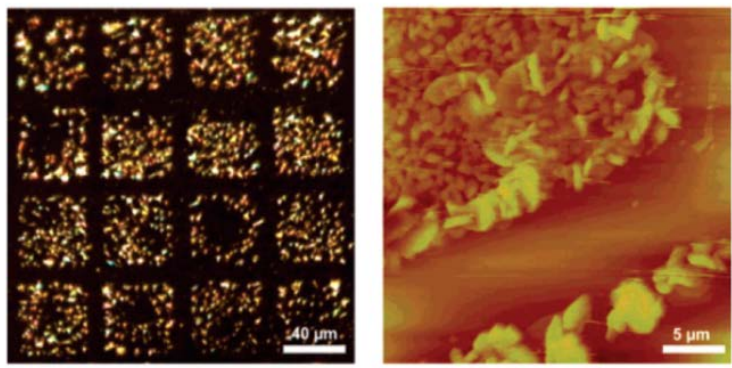

Fig. 1 Optical microscope (left) and AFM (right) images of a laterally 65 patterned MOF-5 film. MOF growth selectively occurs on those areas terminated by a $-\mathrm{CO}_{2} \mathrm{H}$ functionalised SAM but not on the areas terminated by an inert $-\mathrm{CF}_{3}$ functionality. The AFM image clearly shows the boundary between the two areas. (Reprinted with permission from reference 33. Copyright 2005 American Chemical Society)

70 Hermes et al used micro-contact printing to form a grid-like patterned SAM consisting of $-\mathrm{CO}_{2} \mathrm{H}$ terminated squares separated by inert $-\mathrm{CF}_{3}$ terminated lines. After immersion in a precursor solution, randomly oriented micron-sized MOF-5 crystallites were selectively deposited on the $-\mathrm{CO}_{2} \mathrm{H}$ terminated 75 areas as shown in Fig. 1, indicating that the surface must have suitable functional groups for MOF binding. This clearly demonstrates that the functionality terminating the SAM surface is critical to MOF growth, and such facile patterning of surfaces allowing selective MOF deposition is an important step for the so preparation of complex MOF-based devices.

SAM functionality can also direct growth along certain crystallographic directions as demonstrated by Biemmi et al for the growth of HKUST-1 using SAMs terminated by $-\mathrm{CO}_{2} \mathrm{H},-\mathrm{OH}$ and -Me groups. ${ }^{37}$ Surface growth was reported for all three ${ }_{85} \mathrm{SAMs}$, but oriented growth was observed for the $-\mathrm{CO}_{2} \mathrm{H}$ and $\mathrm{OH}$ terminated surfaces as confirmed by X-ray diffraction of the MOF thin films. For $-\mathrm{CO}_{2} \mathrm{H}$ SAMs only peaks belonging to the [100] crystallographic orientation are observed, whereas only peaks associated with [111] are recorded for the -OH terminated 90 surface perpendicular to the substrate. (Fig. 2) The observed orientations for the $-\mathrm{CO}_{2} \mathrm{H}$ and $-\mathrm{OH}$ SAMs correspond to growth along the paddlewheel SBUs and axial sites, respectively.

Related to this is the influence of SAM symmetry on MOF crystal growth. For example, $\mathrm{Fe}(\mathrm{OH})(\mathrm{bdc})$ exists as two 95 polymorphs, a monoclinic phase (Fe-MIL-53) and a hexagonal phase (Fe-MIL-88B) which differ in how the octahedral $\mathrm{FeO}_{6}$ building units are arranged. Preparation of a mother solution of these components yields two products: monoclinic Fe-MIL-53 as a result of homogeneous nucleation during solution aging, and 100 Fe-MIL-88B deposited on a carboxylate-terminated SAM immersed in the same solution. ${ }^{39}$ This remarkable polymorph selection effect is attributed to the inherent approximately hexagonal symmetry of the carboxylate-terminated SAM, which also results in oriented growth along the [001] direction where the 105 six-fold axis of the Fe-MIL-88B lattice is further aligned with the hexagonal symmetry of the organic surface. 

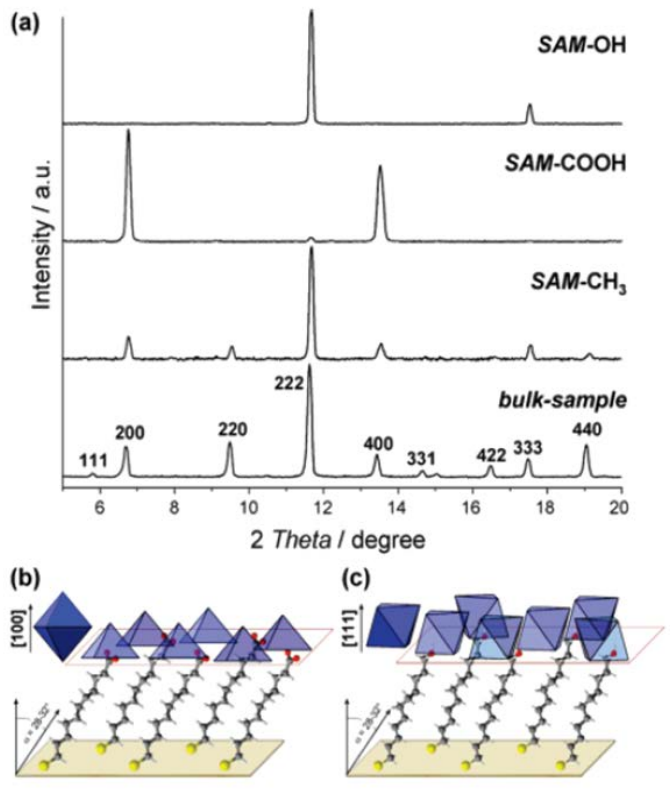

Fig. 2 (a) Background corrected XRD patterns of HKUST-1 thin films grown on different terminated SAMs on Au substrates clearly show SAM-dependent orientation compared to the bulk powder sample. (b) and 5 (c) show schematic illustrations of the observed oriented growth of HKUST-1 along paddlewheels and axial sites on $-\mathrm{CO}_{2} \mathrm{H}$ and $-\mathrm{OH}$ terminated SAMs, respectively. (Reprinted with permission from reference 37. Copyright 2007 American Chemical Society)

An alternative gel-layer strategy has recently been reported by ${ }_{10}$ Schoedel et al, which allows higher concentrations of reactants to be employed and removes the need to precondition reaction solutions. ${ }^{40}$ Here thin poly(ethylene oxide) or poly(ethylene glycol) gel layers are employed to store a high concentration of the metal ions in close proximity to a SAM-terminated surface. ${ }_{15}$ Following diffusion of the linker molecules (ligands) through the metal containing gel, heterogeneous nucleation of the MOF occurs at the gel-SAM interface.

Highly oriented HKUST-1 and Fe-MIL88B- $\mathrm{NH}_{2}$ films were prepared in this way, and for HKUST-1 the observed orientation 20 was independent of SAM terminus. For Fe-MIL88B- $\mathrm{NH}_{2}$ nucleation and growth were influenced by the molecular weight of the polymer (gel) and film thickness by the concentration of Fe(III) in the gels, which led to thicker films and island formation. ${ }^{40}$ Given the diverse range of properties and molecular 25 weights of suitable gel matrices available, it might be expected that the gel-layer synthesis method is widely applicable.

The second mild technique developed for the facile preparation of MOF thin films on SAMs, is a LbL method where the SAM is exposed to the constituent metal ions and ligands separately ${ }^{38}$ as 30 illustrated in scheme 1, rather than in one-pot as described for precursor solutions. This is often referred to as liquid-phase epitaxy, is a bottom-up technique and has since been developed for a number of MOF types composed of stable SBUs, including some containing more than one ligand type. ${ }^{41}$ This method has 35 allowed the preparation of extremely homogeneous atomically flat MOF films, referred to by the portmanteau SURMOFs (SURface MOFs).

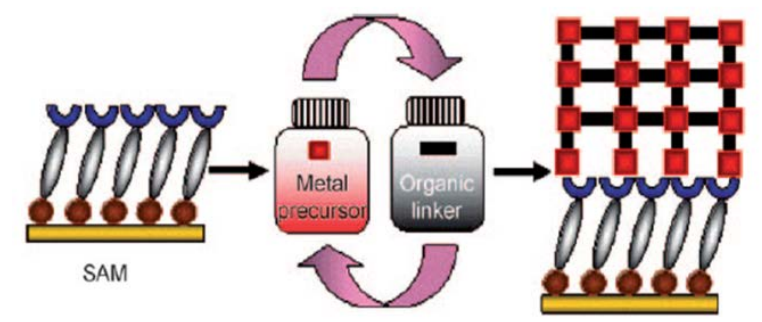

Scheme 1 Illustration of the layer-by-layer (LbL) approach for the growth 40 of MOFs on SAM-functionalised substrates. This strategy involves repeated cycles of immersion of the surface into separate solutions of the metal precursor and organic ligands with a rinsing cycle between. (Reprinted with permission from reference 42. Copyright 2009 Wiley$\mathrm{VCH})$

45 Shekhah et al prepared thin films of HKUST-1 by sequentially immersing a $-\mathrm{CO}_{2} \mathrm{H}$ or $-\mathrm{OH}$ terminated SAM in solutions of the metal ion $\left(\mathrm{Cu}(\mathrm{OAc})_{2}\right)$ followed by the ligand (btc). A rinsing step to remove unreacted components was used between each immersion, and the MOF was gradually built up through 50 repetition of this process. ${ }^{38}$ Film growth was monitored in-situ using Surface Plasmon Resonance (SPR) (an example of this data is shown in Fig. 3) which showed a steady increase in signal (measured in refractive index units, RIUs) with each successive cycle; and after 40 cycles sufficient material was deposited to 55 obtain out-of-plane XRD data. The films were found to be selectively deposited and highly oriented along identical crystallographic directions as those reported for HKUST-1 films prepared from precursor solutions. ${ }^{37}$

In the LbL growth method, the nature of the metal source is an 60 important parameter to successfully prepare homogeneous thin MOF films. This is related to the fundamental SBU approach, which suggests that small clusters (SBUs) of metal ions first assemble in solution and then aggregate through ligand interactions/exchange processes to form the observed open ${ }_{65}$ frameworks. Shekhah et al investigated this by switching the source of $\mathrm{Cu}(\mathrm{II})$ ions from the acetate to the nitrate salt during a LbL assembly of HKUST-1 on both $-\mathrm{CO}_{2} \mathrm{H}$ and $-\mathrm{OH}$ terminated SAMs. ${ }^{42}$

Framework assembly along each direction was followed using ${ }_{70} \mathrm{SPR}$, and in each case growth was retarded when the $\mathrm{Cu}$ acetate was changed for $\mathrm{Cu}$ nitrate as the metal source; growth could however be restarted by switching back to the acetate salt. (Fig. 3) This is explained in terms of the coordination environments of the metal ions in the simple salts and their similarity to, or ease 75 by which SBUs can form from these. For example, $\mathrm{Cu}(\mathrm{II})$ acetate largely exists as $\left[\mathrm{Cu}_{2}(\mathrm{OAc})_{4}\left(\mathrm{H}_{2} \mathrm{O}\right)_{2}\right]$ paddlewheel dimers (Fig. 3 inset) which are identical to the SBUs in HKUST-1, whereas $\mathrm{Cu}(\mathrm{II})$ nitrate contains diverse solvated species. HKUST-1 assembly from $\mathrm{Cu}(\mathrm{II})$ acetate is thus a simple ligand exchange 80 process of OAc for btc in the performed SBU, but is likely more complex for $\mathrm{Cu}(\mathrm{II})$ nitrate systems. This strategy is known as the 'controlled SBU approach'. 


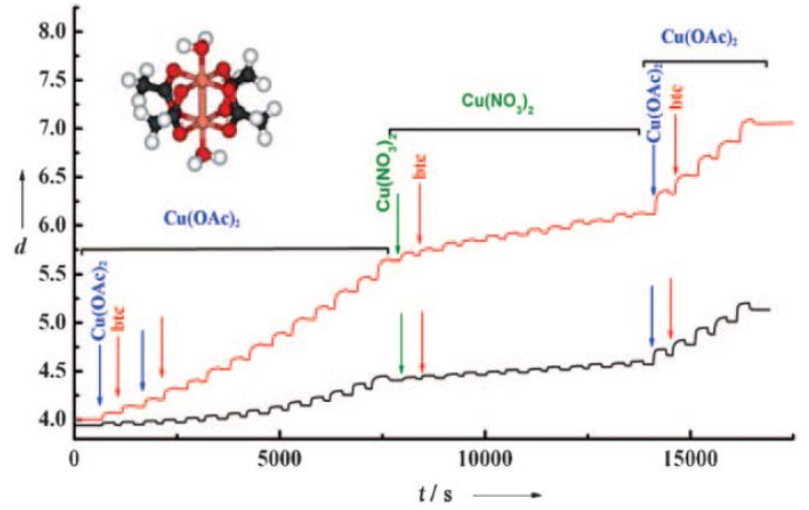

Fig. 3 SPR signal $\left(10^{-2} \mathrm{RIU}\right)$ recorded in-situ as a function of time for successive growth cycles of HKUST-1 on $-\mathrm{CO}_{2} \mathrm{H}$ (red) and -OH (black) terminated SAMs on Au. It is clearly seen that in both cases film 5 thickness increases steadily when $\mathrm{Cu}(\mathrm{OAc})_{2}$ is the metal source, but virtually no growth occurs with $\mathrm{Cu}\left(\mathrm{NO}_{3}\right)_{2}$. It is also noted that film growth rate on the $-\mathrm{OH}$ terminated surface is somewhat slower. (Reprinted with permission from reference 42. Copyright 2009 Wiley$\mathrm{VCH})$

10 LbL growth of MOF thin films is not restricted to MOFs containing one ligand, and has been extended to include multilinker pillared layer systems of general formula $\left[\mathrm{M}_{2}(\mathrm{~L})_{2}(\mathrm{P})\right]$, where $\mathrm{L}$ is a dicarboxylate species and $\mathrm{P}$ is a dinitrogen-based pillaring ligand. ${ }^{41,43}$ In this isoreticular family the carboxylate
15 ligands (L) are linked into (4,4)-connected 2-D grid-like layers via paddlewheel SBUs, which are further cross-linked by the dinitrogen pillars (P) into 3-D open framework structures of high porosity. With an additional component in the system, there are two ways LbL assembly can be effected: (1) use separate 20 solutions for each component $(\mathrm{M}+\mathrm{L}+\mathrm{P})$; or (2) use two solutions, one containing the metal ion and the other a stoichiometric mixture of the two ligands $(\mathrm{M}+(\mathrm{L}+\mathrm{P}))$ for sequential SAM immersion. Thin films of $\left[\mathrm{Cu}_{2}(\mathrm{ndc})_{2}\right.$ (dabco) $]$ (ndc $=1,4$-naphthalene dicarboxylate; dabco $=1,4$ 25 diazabicyclo[2.2.2] octane) have been prepared on $-\mathrm{CO}_{2} \mathrm{H}$ and pyr (pyridyl) terminated SAMs to orient growth along the layer [100] and pillar [001] directions, respectively. Surprisingly, using the $(\mathrm{M}+(\mathrm{L}+\mathrm{P}))$ methodology, ordered growth only occurs along [001] on -pyr terminated SAMs but not along the [100]. By 30 separating all of the components and employing a 3-step $(\mathrm{M}+\mathrm{L}$ + P) LbL process however, ordered growth could be observed along the [100]. (Fig. 4) As might be expected for (M+L + P) LbL growth, the order of addition is important to the assembly process ${ }^{41}$ with highly ordered growth along [100] only observed 35 for the sequence $\mathrm{M} \rightarrow \mathrm{L} \rightarrow \mathrm{P}$. Separation of the linkers in this way can also switch the orientation observed for $\left[\mathrm{Cu}_{2} \text { (ndc) }\right)_{2}$ (dabco)] thin films over the corresponding two-step LbL growth. For example, on -pyr terminated SAMs the expected [001] growth for the two-step process can be switched 40 to [100] using a $\mathrm{M} \rightarrow \mathrm{P} \rightarrow \mathrm{L}$ addition sequence. (a)

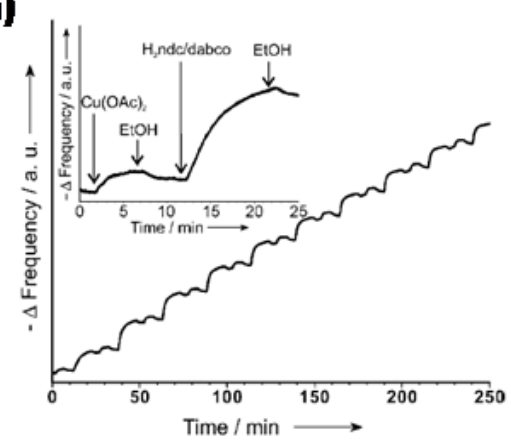

(b)

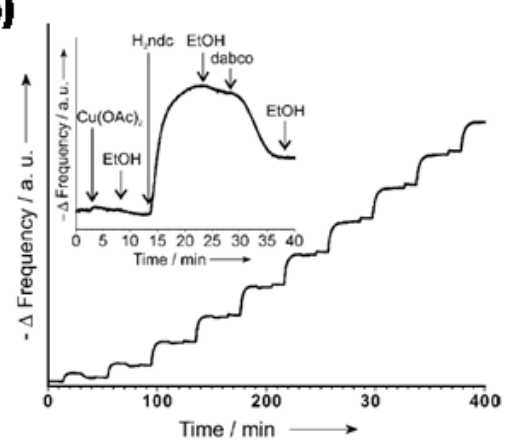

(c)

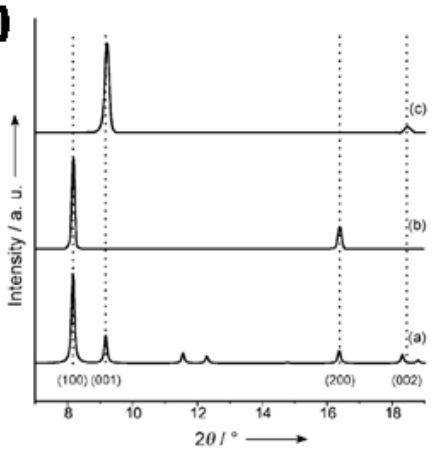

Fig. 4 In-situ QCM monitoring of two LbL experiments of the pillared layer MOF $\left[\mathrm{Cu}_{2} \text { (ndc) }\right)_{2}$ (dabco)] for (a) a two-step $(\mathrm{M}+(\mathrm{L}+\mathrm{P}))$ growth process on a -pyr terminated SAM and (b) a three-step $(\mathrm{M}+\mathrm{L}+\mathrm{P})$ growth strategy on a $-\mathrm{CO}_{2} \mathrm{H}$ terminated surface. (c) shows the background corrected XRD patterns resulting from these growth strategies highlighting the (001) orientation on -pyr terminated SAMs (top) and the (100) orientation on a $-\mathrm{CO}_{2} \mathrm{H}$ terminated 45 surface (middle) compared to the bulk MOF (bottom). (Adapted with permission from reference 41. Copyright $2011 \mathrm{Wiley-VCH).}$

Addition sequence is critical only to the first layers of deposited material, and further addition to a previously deposited layer appears insensitive to the growth strategy employed i.e. an oriented 'seed' layer will direct the orientation of subsequent 50 layers. This permits the preparation of hetero-structured $\left[\mathrm{M}_{2}(\mathrm{~L})_{2}(\mathrm{P})\right]$-type MOF thin films containing different $\mathrm{M}$ and $\mathrm{L}$ components that are reminiscent of the heteroepitaxial growth of MOF@MOF crystals described later in this article. This is an important step for the preparation of ordered MOF thin films with 55 spatially separated and organised hierarchical functionalities.

LbL growth of multilinker MOFs further allows the preparation of materials that are inaccessible using standard bulk synthetic strategies; for example, through control over network interpenetration. $^{43}$ Interpenetration arises when one or more 60 networks occupy the void space of another, and up to 54-fold interpenetration has been recorded. ${ }^{44}$ It is especially prevalent in networks with large pore sizes, is hard to suppress and necessarily limits the porosity of the resulting materials.

Solvothermal synthesis of $\left[\mathrm{Zn}_{2}(\mathrm{bdc})_{2}\right.$ (bipy) $]$ (bipy $=4,4^{\prime}$ 65 bipyridyl), also known as MOF-508, yields doubly interpenetrated pillared layer structures whose polymorphism is dependent on the presence (MOF-508a) or absence (MOF-508b) of included solvent molecules. LbL deposition of MOF-508 on a -pyr terminated SAM using a two step $(M+(L+P)$ ) (where $M$ is 70 a preformed SBU) strategy, favours the growth of an oriented non-interpenetrated and solvent free form of MOF-508a as determined by out-of-plane XRD, IRRAS and $\mathrm{Kr}$ adsorption. ${ }^{43}$ Such a material is not accessible using bulk synthesis, and its observation here can thus be attributed to the nucleation template 75 effect of the functionalised surface and favourable deposition at 
the growing layer suppressing homogeneous nucleation of the second sub-lattice.
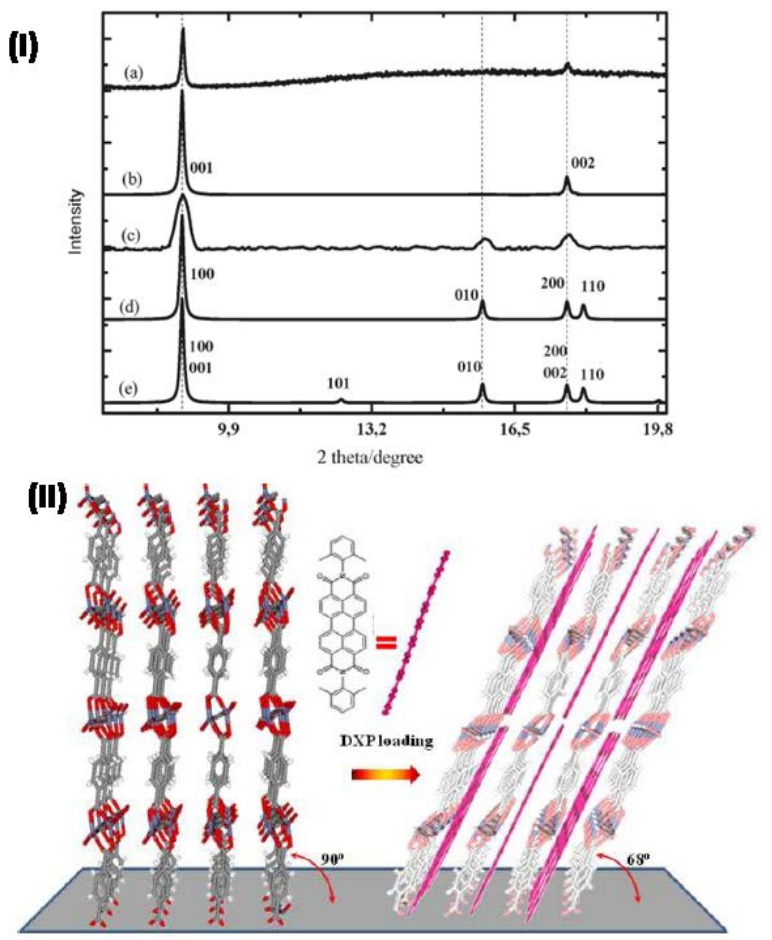

Fig. 5 (I) Experimental (a, c) and calculated (b, d) XRD patterns of the 5 layer-based SURMOF $\left[\mathrm{Cu}_{2}(\mathrm{bdc})_{2}\left(\mathrm{H}_{2} \mathrm{O}\right)_{2}\right]$ for out-of-plane (a,b) and inplane (c,d) orientations compared to the bulk (e). (II) Schematic representation of the structure of $\left[\mathrm{Cu}_{2}(\mathrm{bdc})_{2}\left(\mathrm{H}_{2} \mathrm{O}\right)_{2}\right]$ (left) and the tilting of the layers observed by XRD upon loading with DXP. (Adapted with permission from reference 45. Copyright 2011 American Chemical 10 Society)

Most of the MOFs prepared using the LbL growth strategy on SAMs are 3-D framework structures, and only very few studies have attempted to prepare 2-D layered structures in this way. This is because of the difficulty in controlling the layer orientation 15 with respect to the surface (parallel or perpendicular) and the lack of strong interactions between the layers to promote further heterogeneous nucleation. However, Arslan et al have recently investigated the LbL growth of layered $\left[\mathrm{M}_{2}(\mathrm{bdc})_{2}\left(\mathrm{H}_{2} \mathrm{O}\right)_{2}\right](\mathrm{M}=$ $\mathrm{Zn}(\mathrm{II}), \mathrm{Cu}(\mathrm{II})$ ) systems - analogous to $\left[\mathrm{M}_{2} \mathrm{~L}_{2} \mathrm{P}\right]$ frameworks with 20 axial water in place of the pillaring species - on SAMs and studied the intercalation behaviour of the resulting films. ${ }^{45}$

Growth of $\left[\mathrm{M}_{2}(\mathrm{bdc})_{2}\left(\mathrm{H}_{2} \mathrm{O}\right)_{2}\right]$ readily occurs on $-\mathrm{CO}_{2} \mathrm{H}$ terminated SAMs, and in-plane and out-of-plane XRD analysis of the films reveals the layers are regularly stacked and oriented 25 perpendicular to the surface. (Fig. 5) The perpendicular orientation is readily explained since the $-\mathrm{CO}_{2} \mathrm{H}$ terminated SAM can replace one of the paddlewheel ligands, which will orient growth in this direction as previously observed for HKUST-1 and $\left[\mathrm{M}_{2} \mathrm{~L}_{2} \mathrm{P}\right]$. From the position of the (001) reflections, the interlayer 30 separations are determined to be $5.6 \AA(\mathrm{Zn})$ and $5.8 \AA(\mathrm{Cu})$ for $\left[\mathrm{M}_{2} \mathrm{~L}_{2} \mathrm{P}\right]$ layers held together via hydrogen-bonding interactions. Characterisation of the layers in this way is significant since purely hydrated $\left[\mathrm{Zn}_{2}(\mathrm{bdc})_{2}\left(\mathrm{H}_{2} \mathrm{O}\right)_{2}\right]$ has previously eluded synthesis in the bulk.

35 Heating the films to $240-290{ }^{\circ} \mathrm{C}$ removes all guest solvent and axially-bound water molecules without apparent loss of longrange order. This permits intercalation of large planar aromatic molecules such as the organic dye DXP (synonym for $\mathrm{N}, \mathrm{N}^{\prime}$ Bis(2,6-dimethylphenyl)-perylene-3,4,9,10-tetracarboxylic

40 diimide) into the activated frameworks. ${ }^{45}$ XRD of the intercalation product reveals the presence of an additional diffraction peak, consistent with the tilting of the layers away from the surface normal by $32^{\circ}$ in order to enhance interactions with the included DXP guests. (Fig. 5)

45 The preparation of MOF thin films on SAMs from either precursor solutions or by the LbL method is not detrimental to their porosity, and several of the studies described above have demonstrated loading of the MOF coatings with a range of guest molecules. For example, MOF-5 films can be loaded with 50 organometallic species such as $\mathrm{Cp}$-allyl- $\mathrm{Pd}^{33}$, which can be subsequently reduced to Pd nps as reported for the bulk phase. ${ }^{46}$ Flexible Fe-MIL-88B films were also found to be porous, switching between open and closed forms as a function of DMF loading level ${ }^{39}$ and films of HKUST-1 after 40 cycles of LbL 55 growth still display the expected irreversible ammonia adsorption behaviour due to metal binding of the adsorptive. ${ }^{38}$ Shekhah et al have also demonstrated the porous nature of MOF films through PSM of thin films of $\left[\mathrm{Cu}_{2}\left(\mathrm{NH}_{2} \text {-bdc }\right)_{2}\right.$ (dabco) $]$ using an aminebased coupling strategy, including with large species such as 160 ferrocenylmethylisocyanate. ${ }^{47}$ Further, the ability to easily integrate MOF thin films into quartz crystal microbalance (QCM) devices using SAM methodology $3^{35,48,49}$ has facilitated the quantification of adsorption processes in the composite films permitting characterisation of the porosity of the MOF coatings.

65 Thin films of HKUST-1 have been prepared on standard goldcoated piezo-active QCM substrate crystals (Fig. 6, inset) from precursor solutions ${ }^{49}$ and using the LbL method. ${ }^{48}$ By monitoring the change in vibration frequency of the quartz resonator, the mass change of the MOF film can be precisely determined as a 70 function of guest loading analogous to gravimetric isotherm determination of bulk material on corresponding nanogram samples. The additional advantage of the LbL strategy is the homogeneity and controllable thickness of the resulting films, which permit the measurement of diffusion constants for the 75 adsorption process. ${ }^{48}$

Water adsorption isotherms were measured at different temperatures by Biemmi et $a l^{49}$ for [111] oriented HKUST-1 films prepared by immersion of a gold QCM electrode modified with a -OH terminated SAM into an HKUST-1 precursor 80 solution. At $294 \mathrm{~K}$ a type IV isotherm was recorded as shown in Fig. 6, corresponding to an uptake of $16 \mathrm{mmol} / \mathrm{g}$ at $80 \%$ relative humidity (confirmed by TGA analysis on a bulk sample pretreated at the same humidity level). As the temperature increased the hysteresis observed upon water desorption was also 85 decreased, until a fully reversible isotherm was observed at $343 \mathrm{~K}$ due to increased equilibration kinetics. 


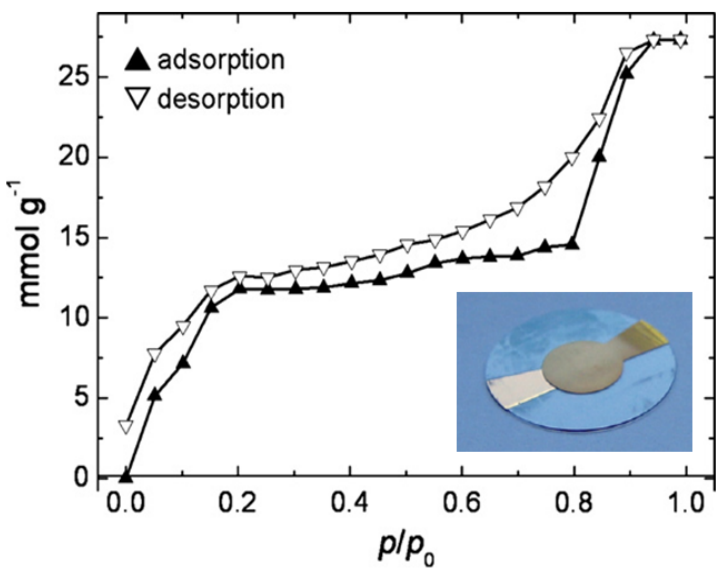

Fig. 6 Water adsorption isotherm measured on a thin film of HKUST-1 at $294 \mathrm{~K}$ with a QCM set-up. The inset shows an opaque layer of the MOF 5 on the SAM-modified gold electrode. (Adapted with permission from reference 49. Copyright 2008 Elsevier)

Similar water uptakes were recorded by QCM devices containing electrochemically deposited HKUST-1 films ${ }^{35}$, indicating that both synthesis methods generate films with 10 comparable physical properties to the bulk MOF. Although water uptake of the HKUST-1 films was cyclable, the long term stability of this MOF to moisture may limit its application in humidity sensing. ${ }^{50}$

QCM devices can also yield information on the time 15 dependence of the mass increase, and for porous materials this kinetic data provides access to diffusion coefficients. For MOFthin films a continuous film of well-defined thickness is required in order to obtain meaningful time dependence analysis of the resulting adsorption curves; consequently, films prepared using 20 the LbL method are best suited to this application.

Zybaylov et al have determined diffusion coefficients for pyridine in [100] oriented $\sim 30 \mathrm{~nm}$ thick HKUST-1 films on QCM substrates. ${ }^{48}$ (Fig. 7) Exposure of activated HKUST-1 films to Argon saturated with pyridine vapour resulted in a rapid increase 25 in real mass density, consistent with binding of the adsorptive to the coordinatively unsaturated axial sites of the paddlewheel SBUs. The mass-uptake saturates after a few minutes corresponding to 11 molecules of pyridine per unit cell, indicating that the pores are not fully occupied at this point.

30 Analysis of the QCM curves using a Fickian diffusion model as shown in Fig. 7 reveals a diffusion coefficient of $1.5 \times 10^{-19}$ $\mathrm{m}^{2} \mathrm{~s}^{-1}$ for pyridine in HKUST-1. The authors further analysed the temperature dependence of the diffusivity by assuming a sitehopping mechanism for guest motion through the porous MOF, to 35 obtain a binding energy of $0.78 \mathrm{eV}$ for the pyridine to the open $\mathrm{Cu}(\mathrm{II})$ sites. This value was only slightly larger than that determined by $a b$ initio calculations.
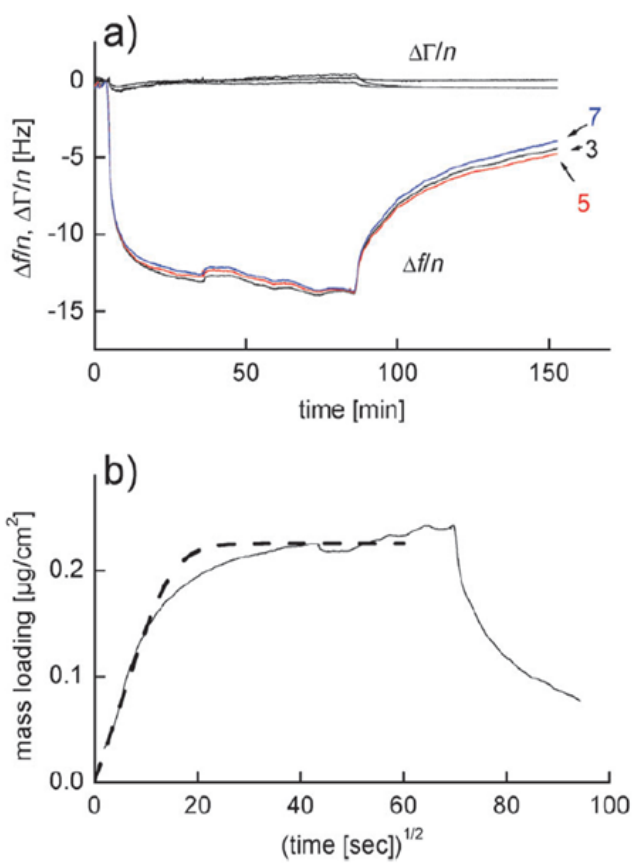

Fig. 7 Data trace of a QCM experiment on HKUST-1 (20 deposition 40 cycles). (a): normalized shifts of frequency $(\Delta \mathrm{f} / \mathrm{n})$ and bandwidth $(\Delta \Gamma / \mathrm{n})$ vs. time. Pyridine is removed from the gas stream at $t=84 \mathrm{~min}$. (b) Mass loading (averaged over the three harmonics shown in (a)) vs. square root of adsorption time. The dashed line is a fit to a Fickian diffusion model. From the slope at small times, a diffusion coefficient $\mathrm{D}=1.5 \times 10^{-19} \mathrm{~m}^{2} \mathrm{~s}^{-1}$ 45 can be estimated. (Reprinted from reference 48 with permission from the PCCP Owner Societies)

In a related study, Uehara et al prepared thin films of monodisperse HKUST-1 nanocrystals of controllable size on modified QCM substrates, which has permitted investigation of 50 the effect of crystal size on the adsorption kinetics of organic vapours in polycrystalline MOF coatings. ${ }^{51}$ Films composed of large 614(11) nm and small 138(1) nm HKUST-1 crystals, denoted L/Au and S/Au respectively, were successfully prepared. (Fig. 8) After activation, the films were exposed to a He gas flow 55 saturated between 0 and $85 \%$ with methanol or hexane vapour. In all cases type I isotherm profiles were recorded, and similar uptakes obtained for both $\mathrm{L} / \mathrm{Au}$ and $\mathrm{S} / \mathrm{Au}$ for each vapour tested.

Vapour adsorption kinetics were determined under low $\left(\mathrm{p} / \mathrm{p}^{0}=\right.$ $\left.0.2 \times 10^{-3}\right)$ and high concentration $\left(\mathrm{p} / \mathrm{p}^{0}=0.85\right)$ conditions, and 60 revealed a clear dependence on crystal size at low concentration independent of analyte species. By contrast, in the high concentration regime an analyte dependency is observed: uptake rates for $\mathrm{MeOH}$ are comparable for both films, whereas those for hexane show a marked difference, and a more pronounced size ${ }_{65}$ dependency than at lower analyte concentration. (Fig. 8) Hexane adsorption kinetics on $\mathrm{L} / \mathrm{Au}$ at $\mathrm{p} / \mathrm{p}^{0}=0.85$ were sufficiently slow to allow the data to be analysed using a multi exponential model yielding kinetic rate constants of 0.00655 and $0.0375 \mathrm{~s}^{-1}$. While this non-linear process remains poorly understood, fundamental 70 studies such as this and those of Zybaylov et $a l^{48}$ are critical if MOF thin films are to be eventually integrated into efficient sensing devices. 


\section{Cite this: DOI: 10.1039/c0xx00000x}
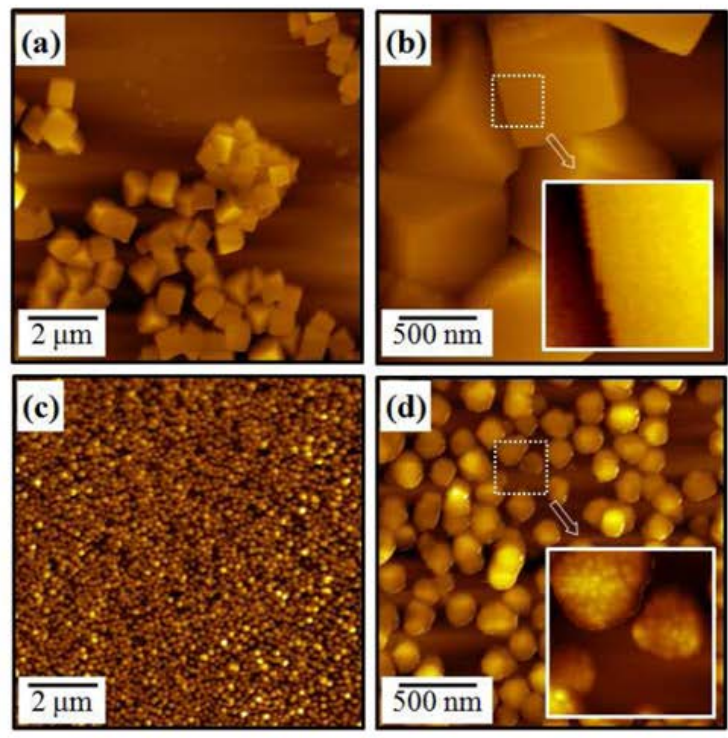
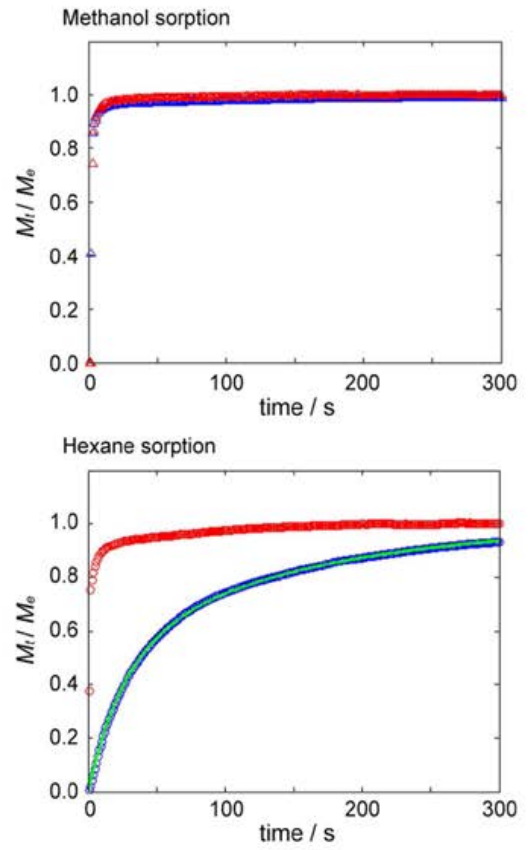

Fig. 8 Left: AFM images of HKUST-1 nanocrystals deposited directly on a Au-modified QCM substrate (a, b) L/Au film composed of $600 \mathrm{~nm}$ crystals and (c, d) S/Au film composed of $130 \mathrm{~nm}$ crystals. Right: Time-dependent mass uptakes of methanol and hexane vapour at $\mathrm{p} / \mathrm{p}^{0}=0.85$ for the two films (L/Au, blue; S/Au, red). The green line for hexande adsorption on L/Au shows how a multi-exponential expression can fit the data. (Adapted with 5 permission from reference 51. Copyright 2011 American Chemical Society)

As outlined in the introduction, the hybrid nature of MOFs gives the network structures an inherent flexibility unique to this class of adsorbent. ${ }^{8}$ Here the crystal lattice of the MOFs can change in response to the number and chemical functionality of 10 included guest molecules; for example, the breathing effects reported for the MIL-n series of compounds where the unit cell volume can change by as much as $300 \% .{ }^{11}$ These guestdependent structural changes can lead to a sensory response by the framework if some other structure sensitive property e.g. 15 photoluminescence, can provide a suitable read-out. ${ }^{52}$ Using a MOF thin film deposited onto an AFM cantilever however, small guest-dependent changes in lattice dimensions arising from molecular adsorption can be probed directly by measuring mechanical stress induced at the interface. ${ }^{53}$

20 Allendorf et al have deposited $100 \mathrm{~nm}$ thick films of HKUST1 on modified piezoresistant microcantilever surfaces using both LbL and single step approaches. Guest-dependent changes in the HKUST-1 lattice were measured for both the hydrated and dehydrated MOF, the latter accessed in -situ by applying a DC 25 voltage across the piezoresistor of the cantilever. In the hydrated state the MOF-coated microcantilever responds reversibly to alcohol and water vapours (Fig. 9) but does not elicit a response to gases such as $\mathrm{N}_{2}, \mathrm{O}_{2}$ and $\mathrm{CO}_{2} .{ }^{53}$ On the other hand the sensor does respond to $\mathrm{CO}_{2}$ when the MOF is in its dehydrated state

30 (Fig. 9, inset), likely arising from strong interactions between the analyte $\left(\mathrm{CO}_{2}\right)$ and the open metal sites of the framework. The response is clearly controllable and sensitive to the chemical nature of the guests: molecules capable of hydrogen-bonding interactions with the framework are detected upon physisorption 35 within the pores in either state, whereas weakly interacting gases are only detected when coordination to open metal sites is permitted via full framework dehydration.

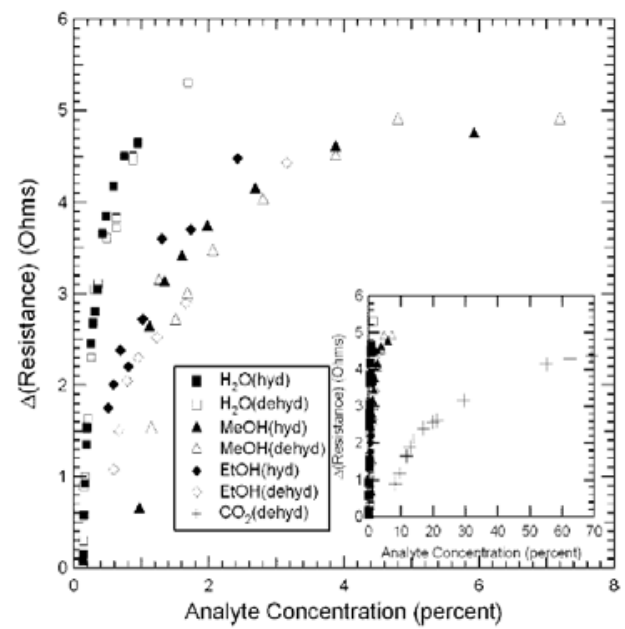

Fig. 9 Resistance change vs. analyte concentration (expressed as a \% of 40 total gas flow in balance $\mathrm{N}_{2}$ ) for HKUST-1 microcantilever devices. The inset clearly reveals that the HKUST- 1 framework responds to $\mathrm{CO}_{2}$ in its dehydrated form. (Reprinted with permission from reference 53. Copyright 2008 American Chemical Society) 
The principle of assembling MOF thin films onto piezoelectric materials to prepare practical integrated MOF-based sensing devices has been extended to metallic nanoparticles and their arrays in order to exploit surface plasmon effects, including 5 localised surface plasmon resonance (LSPR) spectroscopy ${ }^{54}$ and surface-enhanced Raman scattering (SERS). ${ }^{55}$ These techniques rely on the excitation of surface plasmons from typically $\mathrm{Au}$ or Ag nps or planar surfaces and detecting the change in some environmental factor they experience at the interface of that 10 metallic surface. For LSPR very small changes in refractive index (RI) are measured allowing changes in deposited film thickness, density fluctuations and molecular adsorption processes to be monitored, whereas coupling of surface plasmons with vibronic oscillations can enhance otherwise weak Raman signals by up to 1514 orders of magnitude in SERS. Both methods have previously been used, respectively, to monitor LbL growth of MOF thin films on $\mathrm{SAMs}^{38}$, and to study the structure of such films after loading with $\mathrm{Ag}$ nps ${ }^{53}$ when insufficient material prohibits the use of diffraction methods.

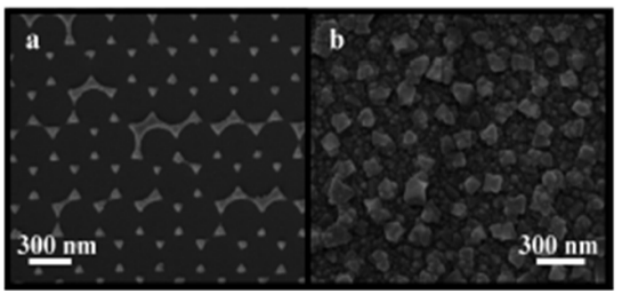

Fig. 10 SEM images of (a) a Ag np array fabricated by nanosphere lithography on a glass coverslip and (b) a Ag np array coated in 20 cycles of polycrystalline HKUST-1 film. (Reprinted with permission from reference 54. Copyright 2010 American Chemical Society)
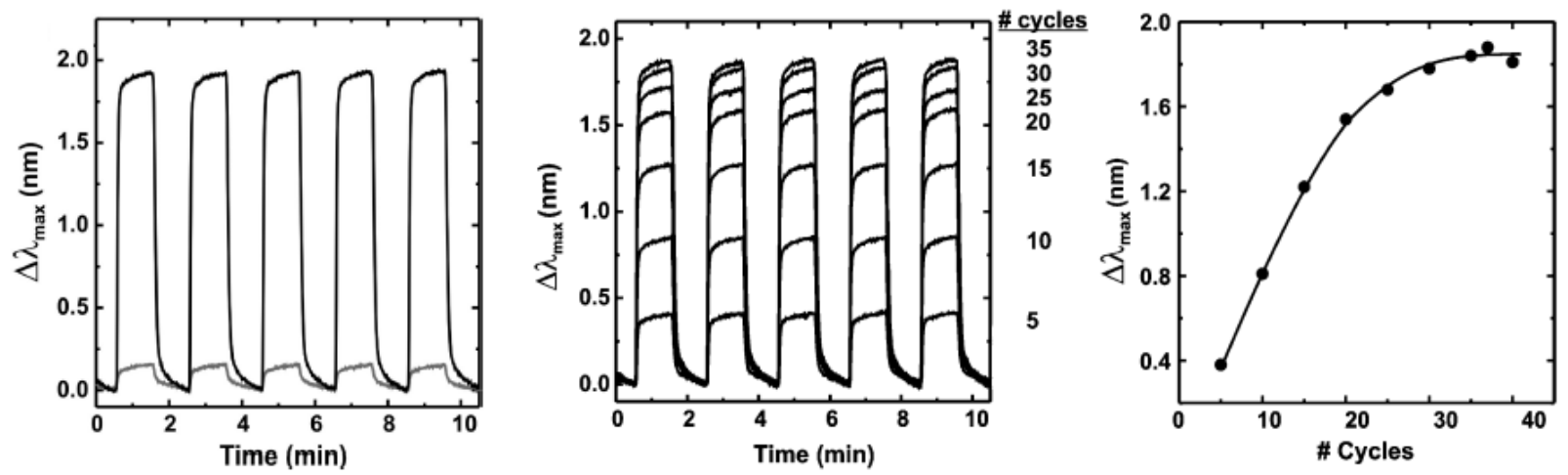

Fig. 11 Left: comparison of the LSPR wavelength shifts $\left(\Delta \lambda_{\max }\right)$ of the HKUST-1/Ag np sensor (black) vs. the bare Ag np array (grey) on adsorption of $\mathrm{CO}_{2}$. Middle: HKUST-1/Ag sensor response to $\mathrm{CO}_{2}$ as a function of the number of growth cycles. Right: Average ( $\left.\Delta \lambda_{\max }\right)$ increases rapidly as a function of MOF thickness at low numbers of growth cycles but begins to plateau around 37 cycles as LSPR response is strongly dependent on distance from the $30 \mathrm{np}$ surface. (Adapted with permission from reference 54. Copyright 2010 American Chemical Society)

LSPR spectroscopy is most widely used for the detection of biomolecular species, where large changes in RI observed upon surface adsorption are more easily discerned than those of small (e.g. gas) molecules where changes in RI can be as small as $10^{-4}$ 35 RIUs. This clearly limits its application in gas and vapour sensing unless sensitivity can be enhanced. However, by employing a $\mathrm{CO}_{2} \mathrm{H}$ functionalised $\mathrm{Ag} \mathrm{np}$ array as a plasmonic substrate and coating this in a HKUST-1 film of controllable thickness using the LbL method, Kreno et al have significantly increased the 40 sensitivity of this technique toward gas molecules. ${ }^{54}$

An array of functionalised Ag nps on a glass substrate was first prepared using standard nanosphere lithography, which was then coated with multiple growth cycles of HKUST-1. The resulting highly crystalline film formed a dense coating over both the 45 particles and the underlying support, with larger crystallites localised on the Ag nps. (Fig. 10) As a reference point, all gas sensing experiments using the MOF-Ag plasmonic composites were also performed with non-functionalised Ag np arrays.

In LSPR spectroscopy shifts in the extinction spectra are 50 measured, and upon adsorption of $\mathrm{CO}_{2}$ a red shift of $\Delta \lambda_{\max }$ by $1.88 \mathrm{~nm}$ is recorded for a composite sensor prepared from 37 HKUST-1 growth cycles. As shown in Fig. 11 (left), this shift is $14 \mathrm{x}$ higher than that observed for the reference array $\left(\Delta \lambda_{\max }=\right.$ $0.13 \mathrm{~nm}$ ) resulting from the enhanced adsorption and thus
55 concentration of the analyte $\left(\mathrm{CO}_{2}\right)$ by the MOF: under the measurement conditions employed, the MOF adsorbs almost 10 times more $\mathrm{CO}_{2}$ than the $\mathrm{N}_{2}$ purge gas. The sensor response is cyclable, able to detect concentrations of $\mathrm{CO}_{2}$ below $10 \%$ and signal enhancement is strongly dependent on the thickness of the ${ }_{60}$ MOF film. (Fig. 11) This is especially prevalent at low numbers $(<30)$ of growth cycles: above this, the observed shifts plateau since the plasmon-induced electromagnetic field decays exponentially from the np surface so only those analyte molecules adsorbed in layers of the MOF closest to the particle ${ }_{65}$ have the largest effect on $\Delta \lambda_{\max }$.

This combination of MOFs with plasmonic np arrays is significant as it is only dependent on the porosity of the framework, so should have broad general application. However, highly specific gas sensing devices could be developed by 70 integrating tailored MOFs with appropriate chemical functionality, pore size or open metal sites to further enhance uptake of one analyte over another.

Raman scattering is enhanced by up to $10^{14}$ times from molecules adsorbed on roughened $\mathrm{Au}$ and Ag surfaces, making 75 this an extremely sensitive technique for the detection of even single molecules thus permitting effective monitoring of trace amounts of chemical and biological species. By incorporating suitably sized metallic nps into MOFs, it should become possible 
to exploit this dramatic signal enhancement to characterise the chemical nature of guest molecules and follow diffusion events through these open network structures in-situ using SERS.

To this end, Sugikawa et al have functionalised high-aspect 5 ratio ( 5) Au nanorods (AuNRs) with a $-\mathrm{CO}_{2} \mathrm{H}$ terminated SAM, and used these as a functional SERS-active interface to prepare MOF-AuNR composites by incubating these in MOF precursor solutions at elevated temperature. ${ }^{55}$ The resulting composites are purple in colour and contain $1.6 \mathrm{wt} \%$ of partially aggregated 10 AuNRs embedded homogeneously throughout the MOF crystals as shown in Fig. 12a,b; the MOF in this case is cubic $\left[\mathrm{Zn}_{4} \mathrm{O}(\mathrm{bpdc})_{3}\right]$ (bpdc $=$ biphenyldicarboxylate) which is isostructural with MOF-5.
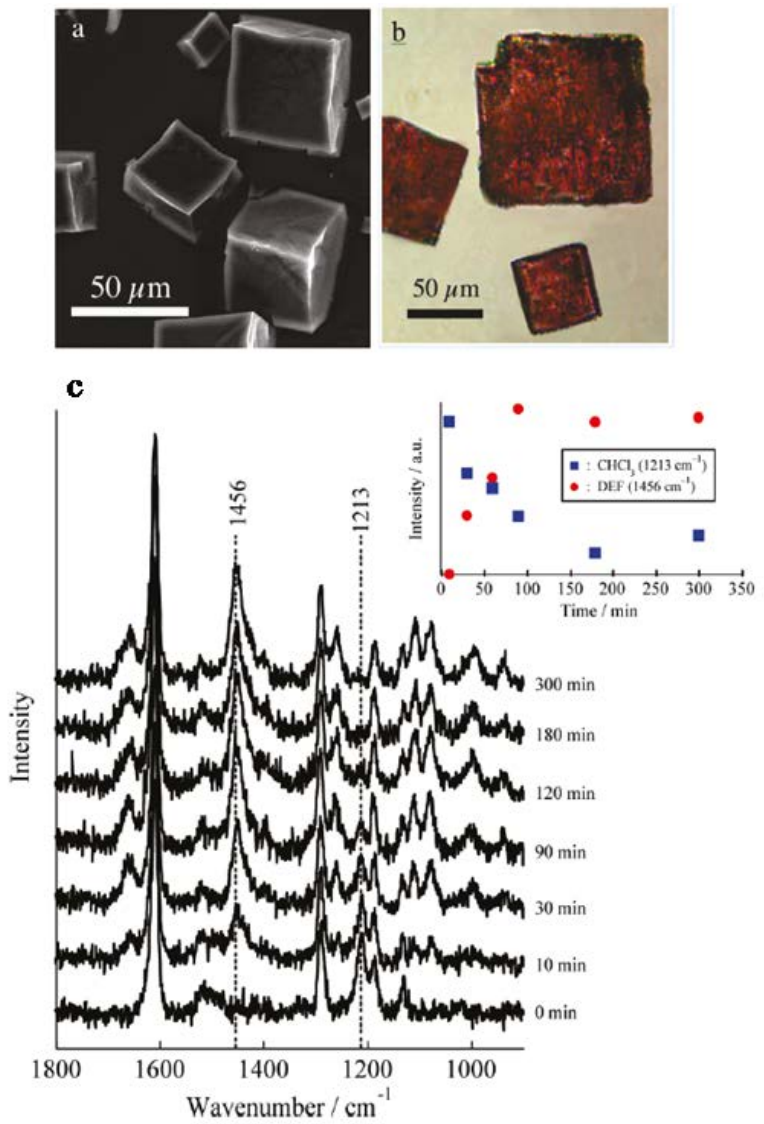

15 Fig. 12 (a) SEM and (b) optical images of MOF-AuNR composite crystals. (c) Time-dependent SERS data following the guest exchange of $\mathrm{CHCl}_{3}$ for DEF in MOF-AuNR composites. The inset shows the changes in intensity of the absorption bands at 1213 and $1456 \mathrm{~cm}^{-1}$ for $\mathrm{CHCl}_{3}$ and DEF, respectively. (Adapted with permission from reference 55. 20 Copyright 2011 American Chemical Society)

The embedded AuNRs in the composite exhibit a characteristic vis-NIR absorption peak at $945 \mathrm{~nm}$ and permanent porosity is maintained, indicating that the composite is ideally configured to sorb guest molecules in the vicinity of the SERS-active AuNRs.

25 Fragments of MOF-AuNR composite crystals were immersed in DEF or $\mathrm{CHCl}_{3}$, and their Raman spectra recorded. In both cases, bands assignable to the guest molecules were clearly observed, and for DEF these were slightly shifted compared to those for the bulk solvent suggesting specific interaction between this guest 30 and the porous MOF. ${ }^{55}$ Using the MOF only as a reference, the intensity of the absorption bands for these guests were significantly weaker confirming the SERS activity of the composite.

Guest exchange of MOFs are important processes, particularly 35 in applications such as drug delivery ${ }^{56}$ or just simply to prevent the partial structural collapse often observed on thermal activation of the porosity. ${ }^{57}$ Such processes have not been widely investigated, but can be followed in-situ using time-dependent SERS spectra. For example, the $\mathrm{CHCl}_{3}$ guest molecules loaded 40 into porous MOF-AuNR composites are successively replaced by DEF over a 100-150 min period. (Fig. 12c) The crystal used in this study was $80 \mu \mathrm{m}$ in width and irradiated at the centre using a laser beam of $5 \mu \mathrm{m}$ diameter. Presumably by adjusting the position of the laser it should become possible to build up a full 45 picture of guest transport dynamics through an individual crystal.

The studies described above for SAMs on Au have provided generic synthetic strategies for the preparation of MOF-based composite materials and revealed the importance of the interface to their nucleation, assembly and orientation. Using these 50 principles a wide-range of functional interfaces has since been employed for MOF growth or deposition, and will be the focus of the remainder of this article.

\section{MOFs at oxide interfaces}

MOFs have been combined with a range of oxide materials to 55 prepare MOF-oxide composites. While a number of configurations have now been prepared, the vast majority of this work involves the often oriented growth of coordination framework membranes onto 2-dimensional oxide surfaces for separation processes. Early efforts in this area were led by 60 Fischer et al. who investigated the solvothermal growth of HKUST-1 and $\left[\mathrm{Zn}_{2}(\mathrm{bdc})_{2}\right.$ (dabco) $]$ onto both bare and functionalised silica and alumina surfaces. ${ }^{58}$ (Fig. 13) Silica surfaces functionalised through covalent silane linkages are significantly more stable than SAMs on Au, permitting direct 65 solvothermal reaction.

On bare substrates, growth was found to be surface selective and highly dependent on compatibility between the acid/base properties of the framework ligands and those of the surface. For example, HKUST-1 which contains only acidic btc linkers was 70 not found to grow on acidic $\mathrm{SiO}_{2}($ IEP $=2.0-2.5)$ but growth did occur on more basic $\mathrm{Al}_{2} \mathrm{O}_{3}(I E P=9.0)$. A similar observation was made for acidic MOF-5. ${ }^{59}$ By contrast, $\left[\mathrm{Zn}_{2}(\mathrm{bdc})_{2}\right.$ (dabco)] grew on both types of (bare) surface resulting from the bifunctional nature of its component ligands. Both MOF-5 and 75 HKUST-1 however, are readily deposited onto $\mathrm{SiO}_{2}$ surfaces modified with $-\mathrm{CO}_{2} \mathrm{H}$ and $-\mathrm{OH}$ functionalities and this can occur in a highly oriented fashion as reported for MOF growth on SAMs on Au substrates ${ }^{20}$; although the orientation on the oxide surfaces appears to be the reverse of that observed on Au.

80 Clearly the growth and density of MOF crystal growth on bare oxide substrates is strongly correlated with surface properties, but which can be tuned through careful selection of reaction conditions. For example, a Ru analogue of HKUST-1 grows on bare oxide surfaces under acidic conditions resulting from the 85 protonation of the surfaces. In the case of $\mathrm{SiO}_{2}$, this would permit hydrogen-bonding between the surface and the carboxylate linker rather than a repulsive electrostatic interaction. ${ }^{60}$ 


\section{HKUST-1}
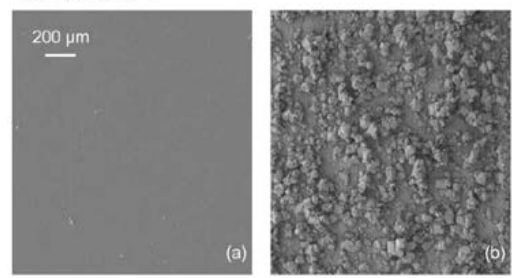

\section{[Zn $\mathbf{n}_{\mathbf{2}}(\mathbf{b d c})_{2}$ (dabco)]}

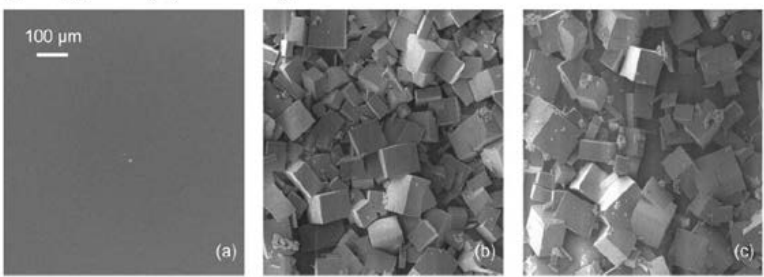

Fig. 13 SEM images of MOF thin films grown on bare and functionalised oxide surfaces by in-situ crystallisation. HKUST-1 (upper images) does not grow on bare $\mathrm{SiO}_{2}$ (a), but does form polycrystalline films on $\mathrm{Al}_{2} \mathrm{O}_{3}$ 5 (sapphire) (b) and $\mathrm{ALD}-\mathrm{Al}_{2} \mathrm{O}_{3}$ (c). For [ $\mathrm{Zn}_{2}$ (bdc) ${ }_{2}$ (dabco)] (lower images), dense MOF coverage occurs on both bare $\mathrm{SiO}_{2}$ (b) and $\mathrm{ALD}-\mathrm{Al}_{2} \mathrm{O}_{3}$ (c) surfaces but not on oxide surfaces terminated with $-\mathrm{CF}_{3}$ groups (a). ALD$\mathrm{Al}_{2} \mathrm{O}_{3}$ is an amorphous $\mathrm{Al}_{2} \mathrm{O}_{3}$ layer deposited onto a silicon wafer using atomic layer deposition. (Adapted with permission from reference 58. ${ }_{10}$ Copyright 2007 Royal Society of Chemistry)

Following these initial studies, MOFs have more recently been grown onto porous oxide surfaces including $\mathrm{Al}_{2} \mathrm{O}_{3}$ and $\mathrm{TiO}_{2}$ for the preparation of separation membranes of high permeances and selectivity, analogous to those reported for zeolites. ${ }^{61}$ By 15 preparing supported MOF-oxide membranes, a new generation of separation systems with superior properties should become accessible arising from the ability to finely tune the pore sizes and functionality presented at the (internal) surface of MOFs. Other favourable properties of MOFs include framework 20 flexibility and non-uniform pore structures. Chiral separation using wholly inorganic membranes has still yet to be achieved, but this also becomes feasible with MOF-based systems, where it is significantly easier to confer chirality on the frameworks through the use of chiral ligands for example. ${ }^{16}$

25 A major requirement for separation systems is the preparation of oriented continuous defect free films so that flow of gas (or liquid) occurs only through the microporous MOF channels, rather than through (macroscale) cracks in the film resulting from inter-crystallite boundaries that will necessarily limit selectivity. ${ }_{30}$ One of the most successful methods to prepare such films utilises a 'seeding and secondary growth' approach, which is employed to favour MOF nucleation on the substrate rather than in solution and has now been applied to a number of MOF types. It is noted that the properties of the seed layers in terms of adherence to the 35 support, seed particle size, anisotropy and orientation, as well as how the support itself has been prepared ${ }^{62}$, are all important factors for the successful preparation of supported MOF membranes.

Films of intergrown HKUST-1 crystals have been prepared on $40 \alpha$-alumina by first spin-coating the support with various seeding suspensions prior to solvothermal synthesis. ${ }^{63}$ Interestingly, seeding the support with a cross-linked layer of a 1-dimensional non-porous coordination polymer of composition $\left[\mathrm{Cu}(\mathrm{Hbtc})\left(\mathrm{H}_{2} \mathrm{O}\right)_{3}\right]$ appears to yield a relatively dense film of 45 phase-pure HKUST-1 after solvothermal treatment in the bulk MOF synthesis solution. The free carboxylate functionality of the 1-D seeds act as both anchoring and nucleation points for the growth of HKUST-1 in the same way as a carboxylate-terminated SAM on Au; however, the crystallites here are randomly oriented 50 on the support which will have consequences for membrane permeance.

For optimum performance of membrane systems, the pore structure of the absorbent (the MOF in this case) should be aligned parallel to the direction of flow to allow maximum flux ${ }^{64}$, 55 which is ultimately dependent on crystal orientation with respect to the substrate. This would have the most pronounced effect on MOF composite membranes prepared from frameworks with 1dimensional pore systems. For example, Arnold et al prepared membranes of $\mathrm{Mn}\left(\mathrm{HCO}_{2}\right)_{2}$, which contains a 1-D pore structure, 60 on both graphite and alumina supports. ${ }^{62}$ Diffraction analysis of the composites revealed the 1-D channel system of the framework was parallel rather than perpendicular to the alumina although the pore system could become better oriented dependent on synthetic conditions.

65 Oriented microporous MOF membranes can be prepared from randomly oriented seed layers. ${ }^{65}$ Tsapatsis and co-workers prepared $20 \mu \mathrm{m}$ thick membranes of $\left[\mathrm{Cu}(\mathrm{L})\left(\mathrm{H}_{2} \mathrm{~L}\right)_{0.5}\right]\left(\mathrm{L}=4,4^{\prime}-\right.$ (hexafluoroisopropylidene)bis(benzoate)) on seeded porous $\alpha$ alumina. Through competitive grain growth during the secondary 70 growth step and conditions favouring growth along the channel axis, the 1-dimensional pore structure of the MOF was found to be aligned perpendicular to the underlying support as confirmed by pole analysis. The oriented composite membrane displayed a high selectivity factor of 23 for $\mathrm{H}_{2} / \mathrm{N}_{2}$ at $190{ }^{\circ} \mathrm{C}$ despite relatively 75 poor permeances. This was attributed to partial blocking of the 1D pores by the seed layer, which implies that MOFs with higher dimensionality pore structures may be better suited for membrane preparation; further, these would not need to be so highly oriented with respect to the chosen support.

${ }_{80}$ In order to form a seed layer that is strongly bound to the support, Hu et al ${ }^{66}$ employed a 'reactive seeding' approach where a porous alumina support acts as the metal $\left(\mathrm{Al}^{3+}\right)$ source in a hydrothermal reaction with the ligand (bdc) component to prepare a MIL-53 seed layer. (Fig. 14) After the secondary growth step a 85 highly intergrown defect free MIL-53 membrane was obtained, as indicated by SEM images (Fig. 14) and the permeation behaviours of small gas molecules which are independent of trans-membrane pressure drop and follow typical Knudsen diffusion behaviour. (Fig. 15) The membrane was also 90 successfully tested in liquid separation: pervaporation studies of ethyl acetate azeotropes demonstrated the water concentration of the permeate could be enhanced from 7 to $99 \%$. This is one of the few examples of liquid separation using MOF-oxide composite membranes, and the selectivity likely arises from the favourable

95 entry of water into the MIL-53 pores which are lined by the terminal hydroxyl groups of the framework SBU. 


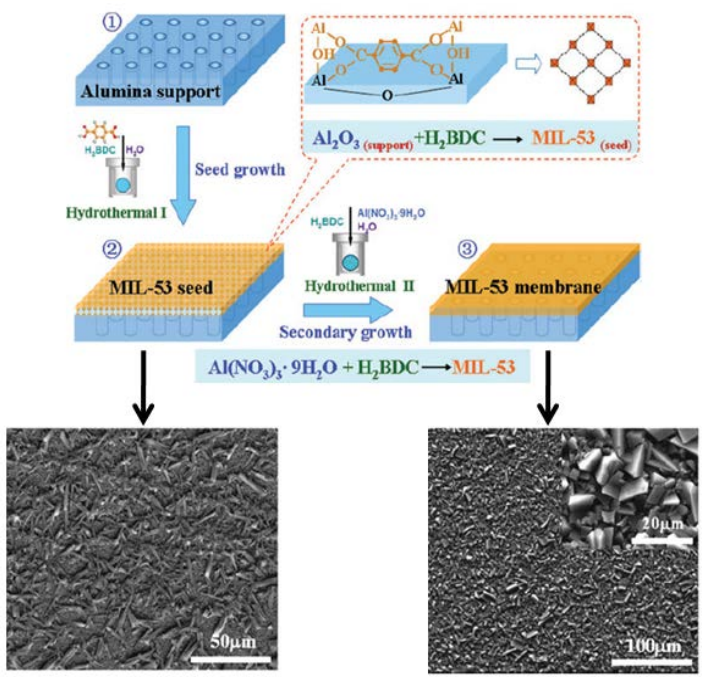

Fig. 14 Schematic representation of the reactive seeding method employed to prepare MIL-53 membranes on porous alumina, where the support acts as the metal source for the preparation of the seed layer,

5 followed by an in-situ growth step. The SEM images show the seed layer (left) and the resulting MIL-53-Al membrane (right). (Adapted with permission from reference 66. Copyright 2011 Royal Society of Chemistry)

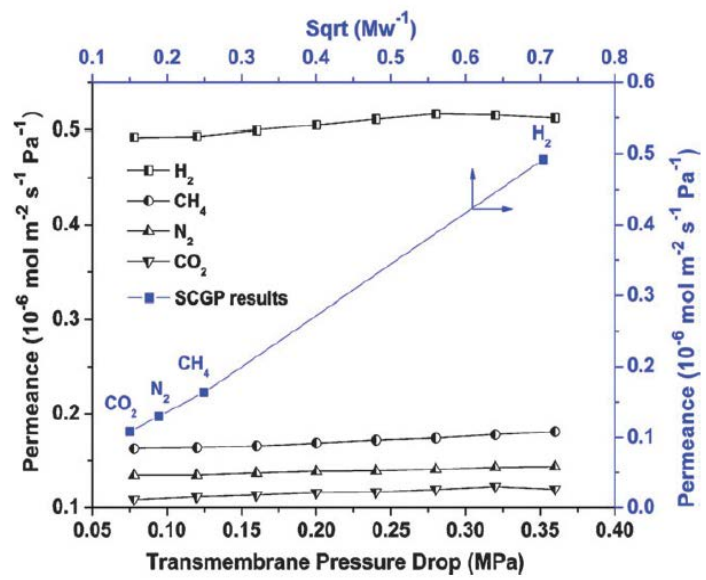

10 Fig. 15 Permeances of small gas molecules at different trans-membrane pressure drops through the alumina supported MIL-53-Al membrane formed by the reactive seeding approach. Single component gas permeation results (SCGP) recorded at $0.8 \mathrm{MPa}$ are also included, which display a linear trend with $\sqrt{ }\left(\mathrm{Mw}^{-1}\right)$ indicating Knudsen diffusion 15 behaviour. (Reprinted with permission from reference 66. Copyright 2011 Royal Society of Chemistry)

HKUST-1 membranes have also been prepared from a preoxidised copper net as the porous support. ${ }^{67}$ Under solvothermal synthesis conditions, the copper oxide mesh provides nucleation 20 sites for homogeneous film growth and an additional source of metal ions to promote adherence to the threads of the support. The HKUST- 1 membrane shows higher permeance for $\mathrm{H}_{2}(1 \mathrm{x}$ $\left.10^{-6} \mathrm{molm}^{-2} \mathrm{~s}^{-1} \mathrm{~Pa}^{-1}\right)$ over other gases $\left(\mathrm{CO}_{2}, \mathrm{CH}_{4}\right.$ and $\left.\mathrm{N}_{2}\right)$, and for $\mathrm{H}_{2} / \mathrm{N}_{2}$ and $\mathrm{H}_{2} / \mathrm{CO}_{2}$ mixtures the separation factors exceed the 25 ideal selectivities.

Tetrahedral Zeolitic Imidazolate Frameworks $(\mathrm{ZIFs})^{68}$ prepared from transition metals (especially Zn(II) and Co(II)) and imidazole-based ligands exhibit a wide-range of zeolite-like topologies and display exceptional chemical and thermal stability, 30 high gas-storage capacity $\left(\text { e.g. } \mathrm{CO}_{2}\right)^{13,69}$ arising from their 3dimensional pore structures and display potential in the separation of alkanes/olefins. ${ }^{70}$ Clearly this robust porosity is an attractive target for the formation of ZIF-based membranes, and there are now an increasing number based on the growth of ZIFs 35 onto porous ceramic supports.

Caro and co-workers have prepared a number of ZIF-oxide composite membranes using both in-situ crystallisation assisted by microwave irradiation and seeding and secondary growth approaches. Often the porous supports are chemically modified 40 with polymers (e.g. polyethyleneimine, PEI) or covalently bound with amino-functionalised silanes (e.g. 3aminopropyltriethoxysilane, APTES) in order to improve adherence and coverage of the ZIF to the ceramic support, as previously detailed for SURMOFs. The resulting ZIF-oxide 45 membranes have been assessed for their permeability, selectivity and molecular-sieving properties toward $\mathrm{H}_{2}$ /gas mixtures, including $\mathrm{CO}_{2}, \mathrm{CH}_{4}$ and propane. a)

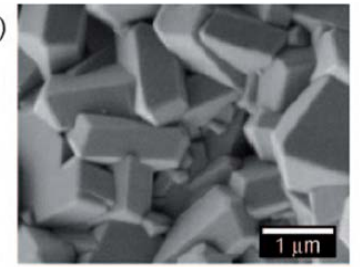

b)

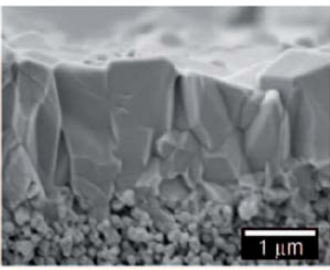

c)

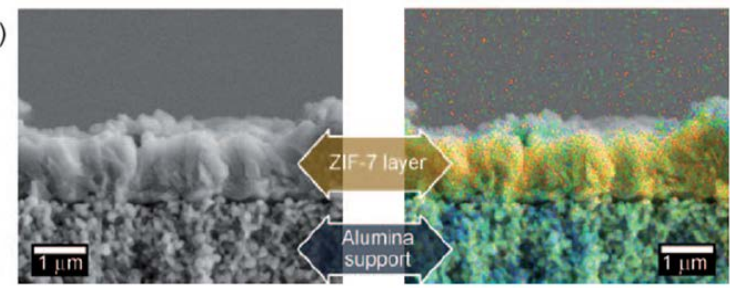

Fig. 16 (a) Top view and (b) cross-section of an alumina supported ZIF-7 50 membrane consisting of a dense layer of intergrown but randomly oriented crystals. (c) EDXS mapping of the ZIF-7 membrane (Zn, orange; Al, cyan). (Reprinted with permission from reference 73. Copyright 2010 Wiley-VCH)

Dense layers of ZIF-8 (2-methylimidazole) ${ }^{71}$, ZIF-22 (555 azabenzimidazole) ${ }^{72}$ and ZIF-7 (benzimidazole) ${ }^{73}$ have all been successfully deposited on titania and alumina supports by in-situ crystallisation, although these tend to be randomly oriented as illustrated in Fig.16 unless the synthetic conditions are carefully controlled. For example, highly oriented ZIF- $7^{74}$ and ZIF-8 ${ }^{75}$ 60 membranes can be grown from randomly oriented seed layers by manipulating the growth kinetics (e.g. through anion and solvent effects and reaction time) during the secondary growth step according to a van der Drift-type evolutionary growth model ${ }^{75}$, where the fastest growing facets that are aligned perpendicular to 65 the substrate dominate.

For all of the ZIF membranes described here, and seemingly independent of topology (SOD for ZIF-7/-8; LTA for ZIF-22), volumetric flow rates indicate that permeances are highly 
dependent on the molecular sizes of the gases with respect to the window size of the $\mathrm{ZIFs}^{71-75}$, with a sharp cut-off observed between $\mathrm{H}_{2}$ and other permeants. (Fig. 17) Of course the membrane thickness and conditions of the experiment $(T, P)$ are 5 also important factors. Window size is unlikely to be limiting however as evidenced by the non-zero permeance of gases with kinetic diameters greater than the framework openings, arising from the modest flexibility of ZIF-type frameworks. ${ }^{76,77}$

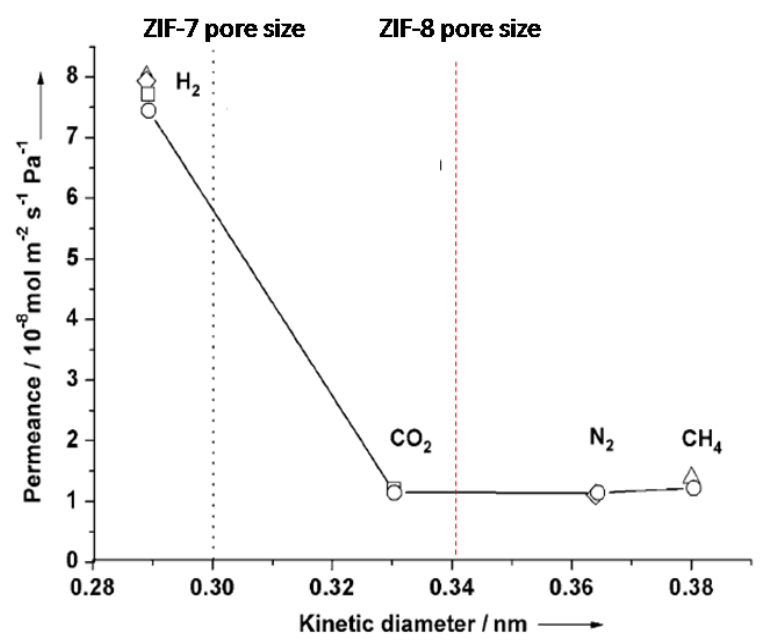

10 Fig. 17 Permeances of single gases (circles) and 1: 1 mixtures $\left(\mathrm{H}_{2} / \mathrm{CO}_{2}\right.$, squares; $\mathrm{H}_{2} / \mathrm{N}_{2}$, rhombuses; $\mathrm{H}_{2} / \mathrm{CH}_{4}$, triangles) at $200{ }^{\circ} \mathrm{C}$ through the alumina supported ZIF-7 membrane shown in fig. 16. The pore sizes estimated from static single-crystal structures for ZIF-7 and -8 are shown for comparison. While this plot shows permeances for ZIF-7, the 15 observed cut-off between $\mathrm{H}_{2}$ and the other gases is representative of most ZIFs studied in this way due to their similar pore sizes. (Adapted with permission from reference 73. Copyright 2010 Wiley-VCH)

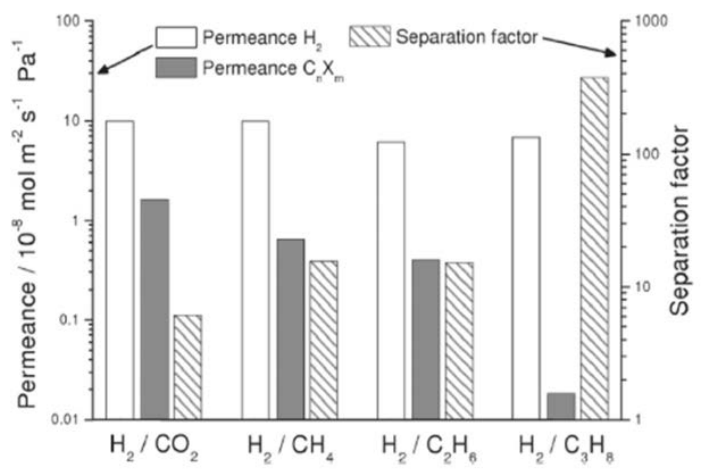

Fig. 18 Permeance and separation factors (logarithmic scale) for 20 equimolar $\mathrm{H}_{2} / \mathrm{C}_{\mathrm{n}} \mathrm{X}_{\mathrm{m}}$ gas mixtures measured at room temperature on an oriented ZIF-8 membrane after a $2 \mathrm{hr}$ secondary growth step. (Reprinted with permission from reference 75. Copyright 2011 American Chemical Society)

In all cases hydrogen permeance (typically on the order of $10^{-}$ ${ }_{25}{ }^{7}-10^{-8} \mathrm{molm}^{-2} \mathrm{~s}^{-1} \mathrm{~Pa}^{-1}$ ) was not found to be adversely affected by the presence of a larger comparatively immobile permeant in 1:1 mixtures due to the advantageous pore structure of ZIFs, and as expected, permeances were increased for the aligned membranes Consequently, separation of $\mathrm{H}_{2}$ from binary mixtures by a 30 molecular sieving effect was much greater than the corresponding Knudsen separation factors; for example, the separation factor for a 1:1 mixture of $\mathrm{H}_{2} / \mathrm{CH}_{4}$ at $298 \mathrm{~K}$ and 1 bar for a ZIF-8 membrane is 11.2 (Knudsen coefficient 2.8) ${ }^{71}$, and selectivities as high as 300 have been observed for $\mathrm{H}_{2} /$ propane mixtures. ${ }^{75}$ (Fig. 35 18)

ZIF-90 contains a 2-caboxaldehyde imidazolate linker that is amenable to post-synthetic modification. ${ }^{78}$ ZIF-90 membranes can be prepared through imine formation between the aldehyde group of the ligand and an APTES modified porous alumina ${ }_{40}$ support. $^{79}$ This is in contrast to ZIF-22 membranes prepared on the same surface which are likely to coordinate through interaction between the $\mathrm{Zn}(\mathrm{II})$ ion and the terminal amino functionality. ${ }^{72}$ The ZIF-90 membranes, once formed, can also be covalently modified by reaction with ethanolamine. ${ }^{80}$ While $\mathrm{H}_{2}$ 45 permeances between the membranes remain relatively unchanged, the $\mathrm{H}_{2} / \mathrm{CO}_{2}$ selectivity factor increases from 7.3 in the unmodified membrane to 62.5 (dependent on activation conditions) following covalent modification. This effect is attributed to a reduction in the pore size of ZIF-90 and healing of 50 defect pores upon imine formation, and demonstrates for the first time that in-situ PSM of MOF-based composite membranes is a realistic and powerful tool by which to tune their selectivity.

More recently, tubular ZIF-oxide composite membranes have also been prepared. Using an in-situ crystallisation method, 55 Aguado et al grew a substituted imidazolate framework (SIM-1) derived from 4-methyl-5-imidazolecarboxaldehyde on $\alpha-\mathrm{Al}_{2} \mathrm{O}_{3}$ tubes of asymmetric pore structure. ${ }^{81}$ SIM-1 has the same SODtopology as ZIF-8. The SIM-1 crystals are highly intergrown and the membrane thickness $(25 \mu \mathrm{m})$ is unchanged along the length of 60 the tubular support.

Permeance data for small gases through the SIM-1-alumina membrane are similar for previously reported ZIF-8 and ZIF-7 membranes as expected, despite the reduction in pore size and volume resulting from the bi-functionalised imidazole linker. ${ }_{65}$ Slightly higher $\mathrm{CO}_{2}$ flux over ZIF-7/-8 membranes is however recorded, attributable to increased interaction between $\mathrm{CO}_{2}$ and the polar aldehyde group in the SIM- 1 pore as evidenced by a higher heat of adsorption. ${ }^{81}$ Separation of $\mathrm{CO}_{2} / \mathrm{N}_{2}$ mixtures is increased from 1.1 based on single permeance results to 4.5 for 70 the ternary mixture $\mathrm{CO}_{2} / \mathrm{N}_{2} / \mathrm{H}_{2} \mathrm{O}(10 / 87 / 3 \mathrm{vol} \%)$, both of which are higher than the Knudsen separation factor. The latter separation is significant due to the humid conditions employed.

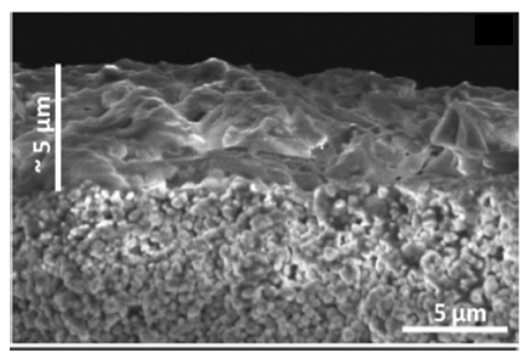

Fig. 19 SEM image of a cross-section of a thin ZIF-8 membrane 75 deposited on a tubular alumina support. (Reprinted with permission from reference 82. Copyright 2010 American Chemical Society)

ZIF-8 membranes have also been prepared on tubular porous alumina using a seeding and secondary growth method, and reported selectivities for $\mathrm{CO}_{2} / \mathrm{CH}_{4}$ mixtures are in the range 4-7, 80 depending on conditions. ${ }^{82}$ Despite this modest separation power, 
extremely high $\mathrm{CO}_{2}$ permeances of $2.4 \times 10^{-5} \mathrm{molm}^{-2} \mathrm{~s}^{-1} \mathrm{~Pa}^{-1}$ were recorded. This is 3 orders of magnitude higher than previous reports for ZIF-8 membranes, and is attributed to the thinness of the membranes (5-9 $\mu \mathrm{m})$ (Fig. 19) and the textural properties of 5 the underlying support. These works clearly suggest tubular ceramics supporting thin MOF films hold significant potential for high permeance high performance composite membrane systems.

Despite the clear advantages of the LbL method for the preparation of highly oriented defect free MOF thin films, this 10 has not generally been applied to the preparation of supported MOF membranes on porous oxide substrates for bulk separation. Perhaps this is an issue of scale, since to prepare an adequate membrane this procedure would necessarily have to be automated, or related to the properties of the oxide surfaces 15 themselves that may not permit the level of coverage experienced for SAMs on Au substrates using this method. However, by integrating the LbL growth strategy with the well developed Langmuir Blodgett (LB) method for the preparation of ordered 2D arrays as shown in Fig. 20, Makiura et al have prepared highly 20 oriented homogeneous thin films of NAFS-1 onto unmodified silica and quartz substrates. ${ }^{83}$
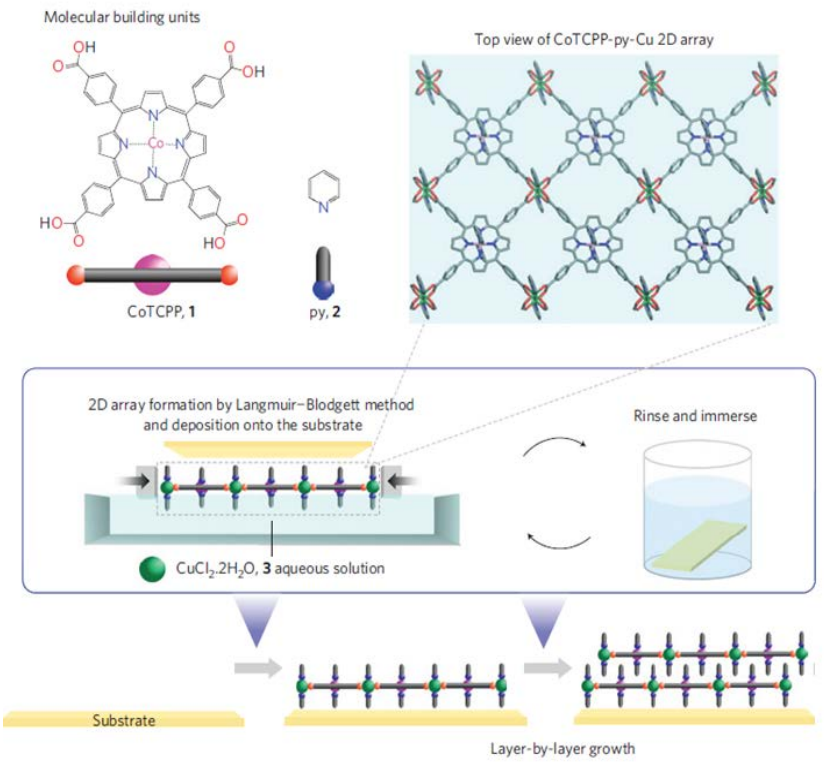

Fig. 20 Preparation of highly-ordered SURMOFs of layer-based NAFS-1 on quartz substrates using a combined Langmuir-Blodgett (LB) and LbL 25 growth method. A seed layer is first prepared at the liquid interface in a Langmuir trough and ordered through compression prior to transfer to the substrate by horizontal dipping. A second LB layer is then transferred onto the first which directs the orientation and allows films of controllable thickness to be easily prepared. (Reprinted by permission from Macmillan 30 Publishers Ltd: Nature Materials (reference 83), copyright 2010)

NAFS-1 is a 2-D MOF based on a $(4,4)$-connected grid of carboxylate-functionalised Co porphyrins (metalloligands) linked via $\mathrm{Cu}$ paddlewheel SBUs, with pyridine bound in the axial positions. (Fig. 20) The growth of NAFS-1 was effected by 35 depositing an organic solution containing the metalloligands and pyridine onto an aqueous solution of $\mathrm{Cu}(\mathrm{II})$ in a Langmuir trough and compressing the surface with barrier walls to form an ordered 'seed' layer which takes the place of a SAM to control orientation of subsequent layers. (Fig. 20)

40 The initial MOF monolayer is easily transferred onto a quartz substrate by horizontal dipping, which was subsequently rinsed and the procedure repeated to prepare films of controllable thickness. ${ }^{83}$ Unusually for this approach a 2-D layer-based MOF is used, where subsequent layers parallel to the surface interact 45 through interdigitation and $\pi$ - $\pi$-stacking of the axially coordinated pyridine groups. This is in contrast to the perpendicularly oriented layers of $\left[\mathrm{M}_{2}(\text { bdc })_{2}\left(\mathrm{H}_{2} \mathrm{O}\right)_{2}\right]$ prepared on $\mathrm{SAMs}^{45}$, and is controllably parallel in the present case due to the synthetic strategy employed.

50 Extensive PXRD analysis of the NAFS-1 films revealed oriented growth and highly crystalline order in both the in-plane (parallel) and out-of-plane (perpendicular) orientations relative to the substrate. This indicates that effective integration of these two bottom-up techniques, permits the same level of control over the 55 in-plane direction as that typically experienced for the out-ofplane orientation in LbL deposited MOF thin films.

While much effort has clearly been dedicated to the preparation of MOF-oxide composite membranes for the separation of gases of strategic importance, MOFs have also been 60 grown on oxide particles which provide further opportunities for applications in separation and catalysis particularly if the particles themselves have some other desirable property e.g. magnetism. For catalysis, processing of the active component into macroscopic shaped bodies in 3-dimensions is usually desirable 65 for ease of handling and recyclability of the active component. This can be achieved by mixing the active with a suitable binder and extruding into pellets for example, or by growing or depositing the catalyst onto a pre-formed shaped support to form easy-to-handle composite materials.

70 SIM-1 has previously been grown onto commercial millimetresized $\alpha$ - or $\gamma$-alumina beads under mild heating conditions to prepare composites containing $10 \%$ of the MOF. ${ }^{84}$ Interestingly, when $\gamma$-alumina is used the majority of the MOF crystals reside within the cavities of the support whereas with $\alpha$-alumina the ${ }_{75}$ MOF crystals form a core-shell type structure covering the alumina bead in a $15 \mu \mathrm{m}$ thick film. (Fig. 21) The reasons for this observation are not clear, but are most likely related to differences in structure and density between the processed alumina polymorphs.

80 The SIM- $1 / \gamma$-alumina composites are particularly attractive for applications as the comparatively fragile MOF crystals in the bead interior are protected by the alumina bead thus enhancing their erosion resistance under mechanical stirring. Using $5 \mathrm{~mol} \%$ SIM- $1 / \gamma$-alumina, the composite beads were found to reduce $90 \%$ 85 of the substrate acetophenone to phenylethanol in the presence of potassium hydroxide after $10 \mathrm{hrs}$ at $80^{\circ} \mathrm{C}$; although the alumina support was itself partially active, control experiments indicated that SIM-1 was clearly the active component of the composite. The composite beads were easily recovered and recycled without 90 loss of activity: an important step for catalytic applications.

Molecular separation using chromatographic methods remains a cornerstone of modern analytical chemistry, allowing chemists to determine the purity of their compounds and assess the efficiency of their catalysts. The tunable pore sizes and 95 functionalities of the $\mathrm{nm}$ sized pores of MOFs are clearly of interest for this application, and a few studies reporting separation using MOFs as chromatographic stationary phases (CSPs) are known ${ }^{85}$, including in single MOF crystals. ${ }^{86}$ In order to improve 
their separation capability, and monodispersity and packing density in chromatography columns however, the preparation of MOF-composite CSPs is emerging as a powerful strategy.
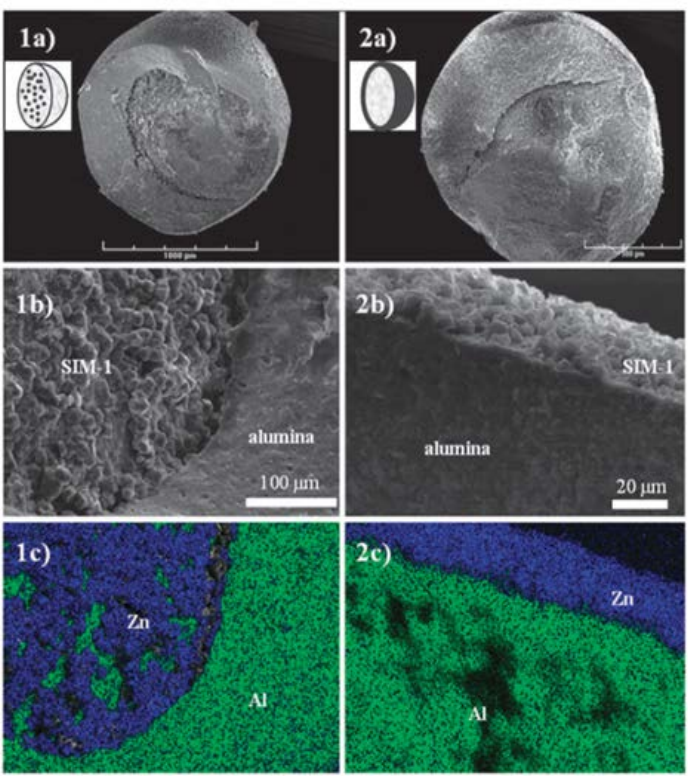

${ }_{5}$ Fig. 21 SIM-1 supported on $\gamma$-alumina (1) and $\alpha$-alumina (2) beads. (a) SEM images of the beads and view of the cross-section. (b) SEM image and (c) EDXS mapping of the core (1) and surface (2). Colour code: $\mathrm{Zn}=$ blue, $\mathrm{Al}=$ green. (Reprinted with permission from reference 84 . Copyright 2010 Royal Society of Chemistry)

10 Ameloot et al have prepared MOF-silica composite particles as stationary phases in liquid chromatography by first impregnating commercial unmodified Nucleosil 100-3 (100 Å pore size, $3 \mu \mathrm{m}$ particles) silica with a HKUST-1 seeding solution, whereby MOF deposition is induced by solvent evaporation. ${ }^{87}$ The loading level 15 of HKUST-1 was 14\% (from TGA) and SEM micrographs demonstrated the uniformity of the composite microparticles. Although the Nucleosil itself is unmodified in this work (i.e. not further functionalised with metal-binding groups), it is a C18 silica where the surface is bonded to non-polar octadecyl 20 functionalities; these are likely to interact with the aromatic ligands to promote MOF growth at the oxide interface or trap small MOF nanocrystals between the flexible alkyl chains.

The HKUST-1-silica composite was slurry packed into a HPLC column and investigated for the separation of ethylbenzene 25 and styrene, in comparison to pure HKUST-1 and standard Nucleosil columns prepared under identical conditions. ${ }^{87}$ (Fig. 22) The HKUST-1-silica column separated the ethylbenzene and styrene extremely effectively with retention times of 4 and 12 min, respectively, with relatively low backpressure. The pure 30 MOF column also separated these components but peaks were broadened due to heterogeneous particle size resulting in significant backpressure, whereas the bare Nucleosil column had much lower backpressure due to smaller and more uniform particle size but no observable resolution. (Fig. 22) This is a clear 35 example of a MOF composite with demonstrable added value over its individual constituents.
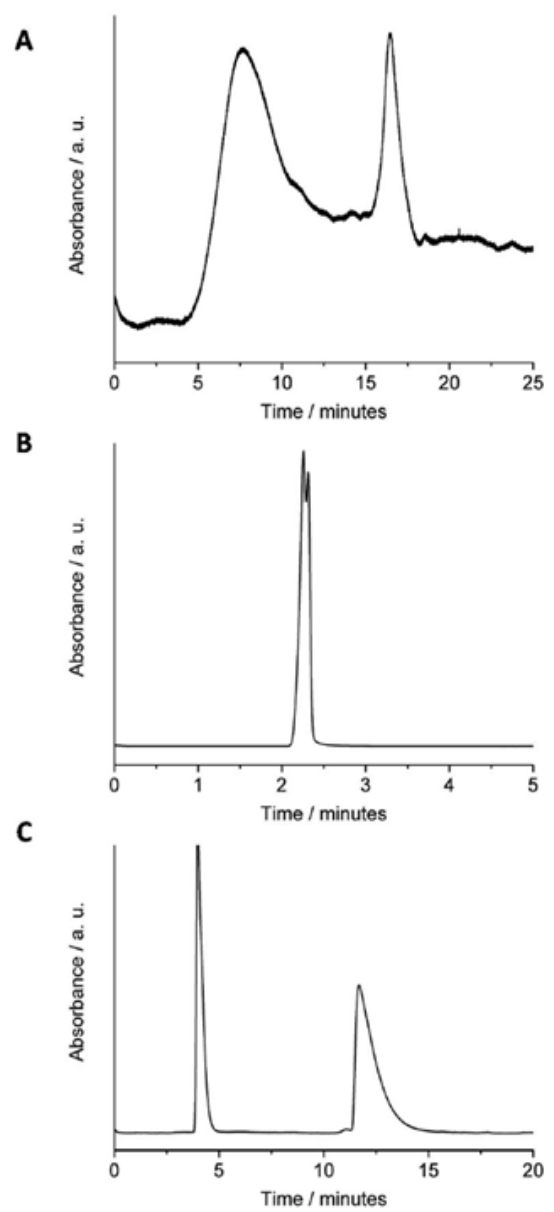

Fig. 22 HPLC experiments for the separation of mixtures of ethylbenze and styrene on (A) a pure HKUST-1 column (B) an unmodified column 40 of Nucleosil-100 silica and (C) the HKUST-1-silica composite column. In chromatogram $\mathrm{C}$, ethylbenzene is eluted first with a retention time of 4.1 min followed by styrene at $12.3 \mathrm{~min}$. The separation factor, $\alpha$, for this mixture is 5.2. (Reprinted with permission from reference 87. Copyright 2010 Wiley-VCH)

45 As noted above, packed columns for liquid chromatography can result in peak broadening (leading to poor resolution) and high backpressures resulting from broad particle size distributions. This is especially true for non silica-based stationary phases where synthesis and packing protocols are less developed.

${ }_{50}$ Further, large quantities of the chosen stationary phase are often required.

To overcome some of the problems associated with packed MOF columns, Gu and Yan prepared a MOF-based gas chromatography (GC) column by surface coating a fused silica 55 capillary column with MIL-101 nanocrystals. ${ }^{88}$ (Fig. 23, upper) The resulting composite GC column had a $0.4 \mu \mathrm{m}$ layer of MIL101 on the inner wall and, after a carefully controlled conditioning programme, allowed baseline separation of $\mathrm{o}$-, $\mathrm{m}$ and $p$-xylenes from ethylbenzene at a temperature of $160{ }^{\circ} \mathrm{C}$ 60 within $1.6 \mathrm{~min}$ of sample injection. (Fig. 23, lower) Amazingly, a total of only $1 \mathrm{mg}$ of the MOF was required to prepare the $15 \mathrm{~m}$ long column (internal diameter $0.53 \mathrm{~mm}$ ), which is significant given the elevated cost of some MOFs - especially those prepared from bespoke ligands - over bulk zeolites and silica 65 phases, making MOF based capillary GC columns a realistic 
alternative. Other isomer mixtures of substituted benzenes and mixtures of $n$-alkanes could also be separated, and extensive control experiments showed that the polarity of MIL-101 combined with its unsaturated metal sites were responsible for the 5 observed selectivities.
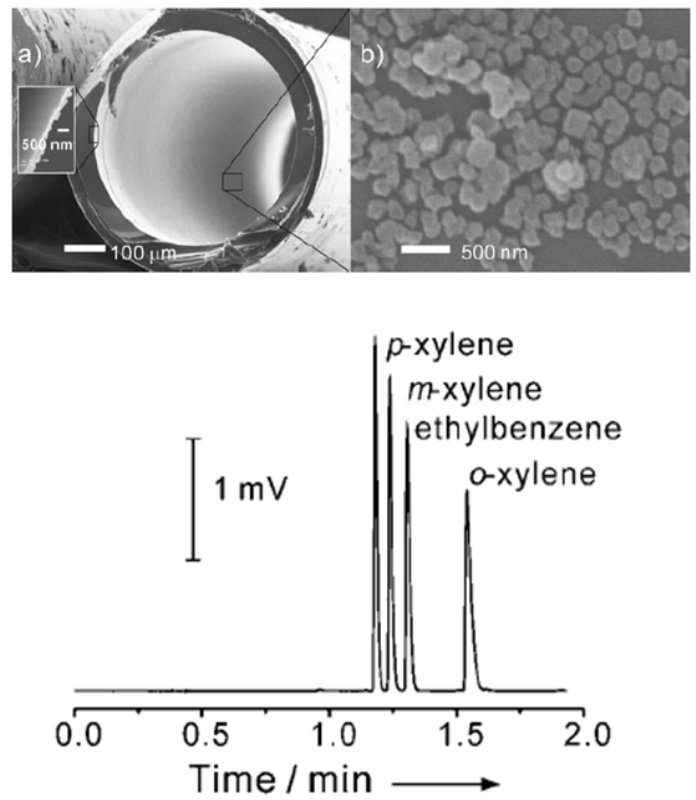

Fig. 23 Upper: SEM images of (a) the cross-section of the MIL-101 capillary column (inset shows thickness of the MOF layer) and (b) MIL101 crystallites deposited on the inner wall. Lower: GC separation of 10 xylene isomers and ethylbenzene by a MIL-101 capillary column (15 m long, i.d. $0.53 \mathrm{~mm}$ ) at $160{ }^{\circ} \mathrm{C}$ under a $\mathrm{N}_{2}$ flow of $3 \mathrm{mLmin}^{-1}$. (Adapted with permission from reference 88. Copyright 2010 Wiley-VCH)

The same group have also used the same dynamic coating method to prepare capillary columns of ZIF-8 for the separation 15 of mixtures of linear and branched alkanes. ${ }^{89}$ The small pore size (3.4 $\AA$ ), sodalite cage structure and hydrophobic surface of ZIF-8 make this ideal for this application, and linear C6 and C8 alkanes are effectively separated from their branched isomers with baseline resolution.

20 Branched alkanes are found to elute much earlier than their linear counterparts on the ZIF-8 column, and do not follow the usual trend of elution which tends to follow the order of boiling points. This arises from a molecular sieving effect where the much bulkier branched alkanes are unable to pass the narrow pore 25 window of the MOF, resulting in shorter retention times overall. Elution time and the observed asymmetric peak shapes for branched alkanes are largely dependent on the length of the linear part of the molecule before the branch point: these carbon chains can enter the pore windows, which leads to delayed elution for 30 longer chains due to increased hydrophobic interactions. The elution order observed for linear and branched alkanes fits with adsorption enthalpies calculated from van't Hoff plots. ${ }^{89}$

Although branched alkanes are not particularly well separated from each other, the ZIF-8 columns do display high resolution 35 and selectivity for the separation of mixtures of linear alkanes covering a broad range of boiling points, with the elution sequence following the order of boiling points. This selectivity arises due to van der Waals interactions between the alkanes and the hydrophobic inner surface of the ZIF-8 micropores. The 40 power of this column was further demonstrated by separating the linear and branched alkane and olefin components of a commercial petroleum sample.

This methodology has recently been extended by Xie et al, who have prepared chiral capillary GC columns of a MOF ${ }_{45}$ derived from a modified amino-acid. ${ }^{90}[\mathrm{Cu}(\text { sala })]_{\mathrm{n}}$ (sala $=\mathrm{N}$-(2hydroxybenzeyl)-L-alanine) is a thermally stable threedimensional homochiral MOF formed by the dehydration induced cross-linking of single-handed helices of $2.5 \mathrm{~nm}$ pitch. The composite column was prepared by dynamic coating a tubular 50 fused silica column, resulting in a $1 \mu \mathrm{m}$ thick [Cu(sala) $]_{\mathrm{n}}$ film on the inner wall. McReynolds constants indicated the MOF columns were of moderate polarity and these efficiently separated test mixtures of alkanes and alcohols as well as positional isomers including those of xylene. ${ }^{90}$ More significantly, the column $(2 \mathrm{~m}$, 55 i.d. $75 \mu \mathrm{m}$ ) could resolve racemic mixtures of wide-ranging functionality - including amino acids - with baseline resolution in most cases. Separation factors were in the range $1-1.3$, and all retention times were $<1 \mathrm{~min}$. Despite its 3-dimensional structure, $[\mathrm{Cu}(\mathrm{sala})]_{\mathrm{n}}$ itself is non-porous so chiral recognition in this case is 60 restricted only to the crystallite surface. It is thus interesting to speculate on the potential of large-pore homochiral MOF materials ${ }^{91}$ in enantioselective GC separations.
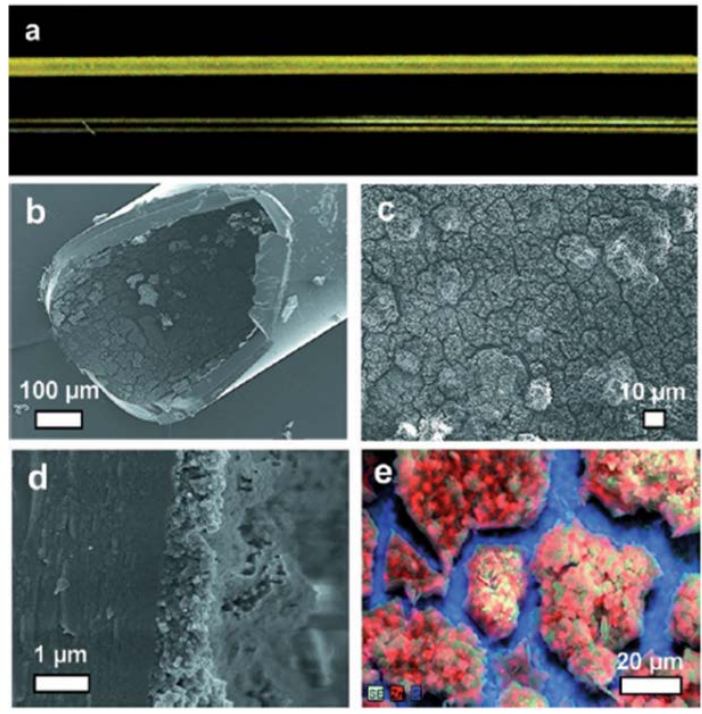

Fig. 24 SEM images of a MOF-5 coated GC fused-silica bonded PLOT 65 capillary column: (a) photograph of a coated (above) and a non-coated (below) capillary; SEM images of (b) cross-section of a coated capillary; (c) a typical layer inside the coated fused-silica column; (d) cross-section of the transition between the capillary wall and the MOF-5 coating. (e) EDX mapping of a typical section of the prepared MOF-5-CSA layer. 70 Blue: areas with high Si content, red: areas with high $\mathrm{Zn}$ content. (Reprinted with permission from reference 92. Copyright 2011 Wiley$\mathrm{VCH})$

The MOF-based capillary GC columns described above were all prepared by coating the interior surface of open tubular oxide 75 columns with pre-formed MOF nanocrystals. Over time it is expected that small particles of the MOF coating will detach from the support leading to spiking phenomena if a particle filter is not used. One strategy to prevent this is the formation of 'bonded 
PLOT' (porous layer open tubular) columns where the coating is chemically crosslinked to the underlying support. Applying this to MOF-based capillary columns, Münch et al have recently combined SAMs and the LbL growth strategy for the preparation 5 of an analogous MOF-5 'bonded PLOT' system for chromatographic separation. $^{92}$

A carboxylate-terminated SAM monolayer was first formed on the internal surface of a commercial fused silica capillary column, and metal and ligand solutions were then successively pumped 10 through the column for 80 growth cycles to prepare a $1 \mu \mathrm{m}$ thick coating comprised of $100-200$ nm MOF-5 crystallites on the functionalised bonded surface. (Fig. 24) The columns were characterised by SEM, EDX mapping and porosity studies and the MOF-5 structure was confirmed by XRD of a MOF coating 15 prepared on a planar substrate under the same conditions, agreeing well with previous studies by Fischer and co-workers. ${ }^{59}$

The MOF-5 capillary was tested for its efficacy in natural gas separation and benchmarked against a commercial $\mathrm{Al}_{2} \mathrm{O}_{3}$-based PLOT column. Each column was run under its optimal operation 20 conditions, and while both separated all five components of natural gas (composition: 97.1\% methane, 1.7\% ethane, $0.7 \%$ propane, $0.2 \%$ isobutane, $0.3 \% \quad n$-butane) with baseline resolution, the MOF-5 column was $15 \%$ faster than the $\mathrm{Al}_{2} \mathrm{O}_{3}$ reference column. Multidimensional spider diagrams as shown in
25 Fig. 25 were constructed by plotting standard chromatographic performance indicators to quantitatively compare the two columns. $^{92}$

Chromatographic resolution between ethane/propane and iso/n-butane were better for the MOF column, but for 30 propane/isobutane the reference column was superior; however, given the similar chromatogram length for both columns, this could simply be related to poorer performance between iso $/ n$ butane. The MOF column also displays less tailing and peak broadening than expected from its micropore structure and has a 35 more uniform distribution between the analytes than the reference, so appears to perform slightly better than the commercial $\mathrm{Al}_{2} \mathrm{O}_{3}$-based PLOT column. ${ }^{92}$ (Fig. 25) Further, the MOF-5 column carried out 300 separations over a five month period without apparent loss of performance.

40 This is the first time such a detailed comparison has taken place and is clearly important to the feasibility and development of MOF-based columns, as well as a means to express their relative merits (or otherwise) over existing separation systems. The authors also investigated important operational parameters 45 such as temperature and flow speed through the column, and determined diffusion coefficients and plate heights from these using van Deemter and Golay plots.
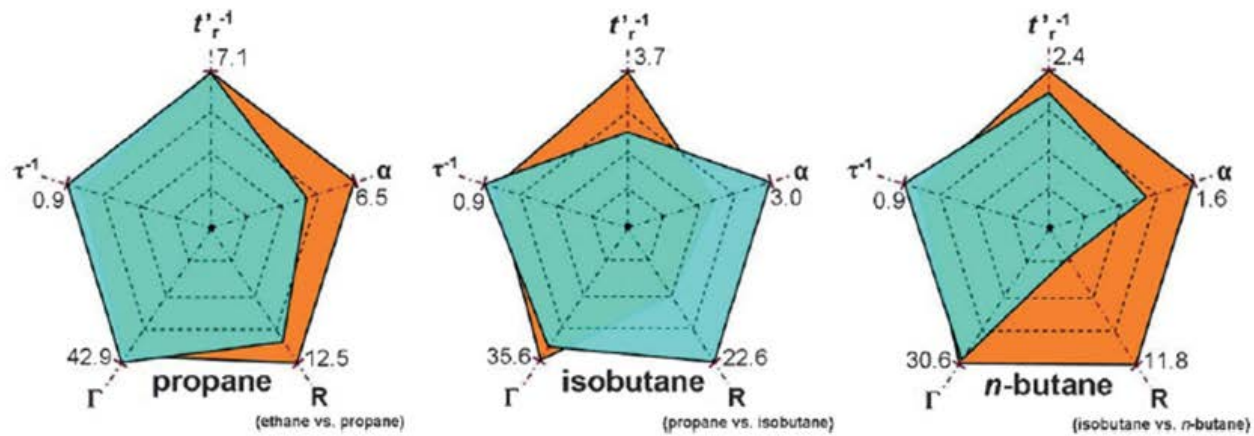

Fig. 25 Spider diagrams of the separation performance parameters of the MOF-5 bonded PLOT column (orange) and a commercial HP-PLOT GC column 50 (blue) for the analytes propane, isobutane, and $n$-butane. The diagrams allow a direct multidimensional comparison of the performances of the two columns. Methane serves as a reference compound and correspondingly ethane is regarded as the first component that truly interacts with the stationary phase. Since the determination of the chromatographic resolution requires the difference of the retention times of two adjacent peaks that are considered to truly interact with the stationary phase, it is only possible to calculate the corresponding values for methane and ethane. Consequently, spider diagrams for these two analytes cannot be generated. Higher values indicate better performance. For this reason, $\tau$ and $t_{\mathrm{r}}$ are expressed by their reciprocal values $\left(t_{\mathrm{r}}\right.$ : 55 reduced retention time $\min ^{-1}, \alpha$ : selectivity factor, $R$ : chromatographic resolution, $\Gamma$ : global resolution factor, $\tau$ : tailing factor). The labels at the bottom indicate the compounds used for the determination of the corresponding $R$ and $\alpha$ values. (Reprinted with permission from reference 92 . Copyright 2011 Wiley-VCH)

The templated hexagonal mesopores of MCM-41 silica have also been investigated as a support for HKUST-1 growth. ${ }^{93}$ 60 MCM-41 was first impregnated with $\mathrm{Cu}(\mathrm{II})$ ions, and the $\mathrm{Cu}$ MCM-41 precursor used as the metal source in a solvothermal reaction with the btc ligand. The resulting composite however did not contain any crystalline HKUST-1, and TGA confirmed that the observed 2:1 ratio of Cu:btc did not match that of the MOF 65 (3:2). While it is not clear what the nature of the metal-organic component is, the similarities in the sorption properties between MCM-41 and the reported MO-MCM-41

(MO = metal-organic) indicate this is unlikely to be a microporous framework. Despite this, the MO-MCM-41 70 composite has both uncoordinated metal sites and free carboxylate groups for interaction with guest molecules, leading to an increased ammonia adsorption capacity versus pure MCM41 and bulk HKUST-1 phases after hydrothermal conditioning.

Magnetic iron-oxide nanoparticles (nps), both surface75 functionalised and bare, have been used to prepare MOF composite materials for a range of applications that can exploit the high porosity and functionality of the MOF and the magnetic properties of the included nps. ${ }^{94-96}$ The composites themselves consist of MOF crystallites with (aggregates of) $\mathrm{Fe}_{3} \mathrm{O}_{4}$ nps ${ }_{80}$ embedded within. Further, the $\mathrm{Fe}_{3} \mathrm{O}_{4}$ nps also constitute a preformed surface for MOF growth.

Sweigart and co-workers reported the exchange of oleylamines on capped $\mathrm{Fe}_{3} \mathrm{O}_{4}$ nps with the complex $\left[\left(\eta^{5}-\right.\right.$ semiquinone)Mn(CO) $)_{3}$, SQMTC, that subsequently acts as a 85 nucleation point for the growth of the non-porous 1-dimensional 
benzoquinone coordination polymer $\left[\mathrm{M}(\mathrm{QMTC})_{2}(\mathrm{dmso})_{2}\right](\mathrm{M}=$ $\mathrm{Mn}(\mathrm{II}), \mathrm{Cd}(\mathrm{II}))$ from the surface of the particle. ${ }^{94}$ All the prepared composites were attracted to a magnet, and [Mn(QMTC $\left.)_{2}(\mathrm{dmso})_{2}\right]-\mathrm{Fe}_{3} \mathrm{O}_{4}$ was found to behave as a 5 superparamagnet in the field range $-2500-+2500 \mathrm{G}$ in contrast to the bulk coordination polymer which follows simple paramagnetic behaviour.
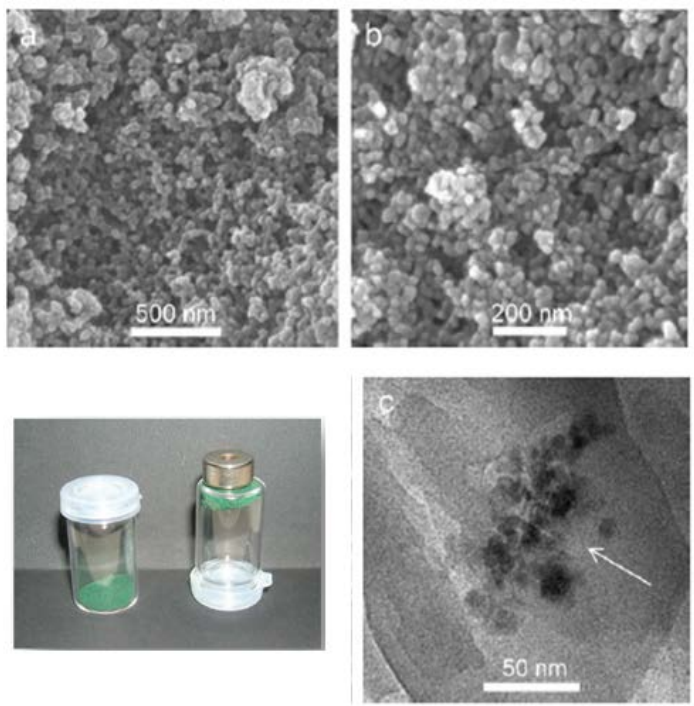

Fig. 26 SEM images of HKUST-1-magnetite composite (a) and a pure 10 HKUST-1 reference sample (b). (c) TEM image of DUT-5- magnetite composite showing the included magnetic nps (arrowed). The photograph shows the HKUST-1 composite held by a small permanent magnet. (Adapted with permission from reference 95. Copyright 2011 Royal Society of Chemistry)

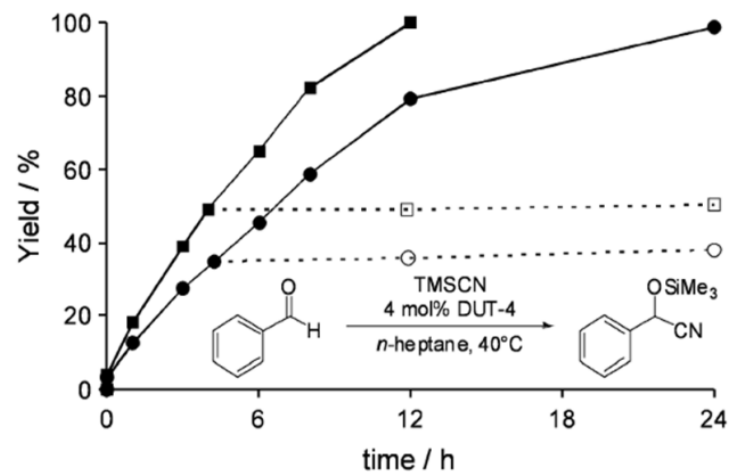

Fig. 27 Product yield during cyanosilylation reaction of benzaldehyde using $4 \mathrm{~mol} \%$ pristine DUT-4 (a) and $3.3 \mathrm{~mol} \%$ magnetically functionalized DUT-4 $(\bullet)$. The open symbols and the dotted lines represent the yield after separation of the catalyst by either filtration or 20 magnetic separation. (Reprinted with permission from reference 95. Copyright 2011 Royal Society of Chemistry)

Recent work by Kaskel and co-workers has demonstrated the versatility of MOF iron-oxide composites in applications including catalysis and drug delivery. ${ }^{95}$ The highly porous MOFs 25 DUT-4, DUT-5 and HKUST-1 were deposited onto uncapped superparamagnetic nps by exploiting the strong interaction between the carboxylate groups of the framework linkers with the magnetite surface. (Fig. 26) Formation of the composites was neither detrimental to the properties of the MOF (e.g. porosity,
30 Lewis acidity) or the superparamagnetism of the iron-oxide, which accounted for $5-15 \%$ of the composite. For example, DUT-4- $\gamma-\mathrm{Fe}_{3} \mathrm{O}_{4}$ was found to catalyse the cyanosilylation of benzaldehyde in quantitative yield after 24 hrs, and was easily recovered using a permanent magnet and much more readily than 35 the bulk DUT-4 control which required filtration. (Fig. 27)

Magnetic particles are currently under intensive study for their biomedical applications. $^{97}$ For example, a magnetic particle provides a means of efficient localisation by an external field into the biological tissue of interest for both therapeutics (drug 40 delivery) and diagnostics (e.g. MRI). The phenomenon of magnetic hyperthermia is also exploited in cancer therapy, where magnetic particles can be heated in an alternating (external) magnetic field to destroy malignant tumours. ${ }^{97}$ By combining the properties of superparamagnetic particles with the demonstrable 45 potential of MOFs in drug delivery ${ }^{28,98}$ through their high drug loading, biocompatibility and controlled release kinetics, multifunctional composite materials for targeted drug delivery, combination therapy and imaging become accessible. Recently, magnetic HKUST-1 composites have been investigated to this 50 end. ${ }^{95,96}$

Placing HKUST-1 iron-oxide composites in an alternating magnetic field leads to externally triggered heating corresponding to a specific absorption rate of $105.7 \mathrm{Wg}^{-1}$ based on the observed iron content ${ }^{95}$, which is close to optimal for biomedical 55 applications. As expected, the release rate of ibuprofen from the composite is temperature dependent, and increasing the temperature from 20 to $40{ }^{\circ} \mathrm{C}$ increases this rate by $50 \%$. This implies that an external magnetic field could be used to tune the rate of drug delivery from MOF-iron-oxide composites, although 60 this currently remains to be demonstrated.

HKUST-1 composites prepared from $\mathrm{Fe}_{3} \mathrm{O}_{4}$ nanorods loaded with the anticancer drug Nimesulide (NIM) have also been tested as potential drug delivery agents. NIM release kinetics were reported, and the composites display magnetic hysteresis loops at $65300 \mathrm{~K}$ with saturation magnetisation values in the range $0.9-1.5$ emu $\mathrm{g}^{-1}(10 \mathrm{~T})$ depending on Fe content, indicating their potential in targeted delivery systems. ${ }^{96}$

MOF-oxide composites have also been used as selective vapour and gas sensing devices as reported for MOFs prepared on 70 functionalised Ag and Au np surfaces and arrays. ${ }^{54,55}$ One of the major barriers for the preparation of effective sensing devices is to appropriately configure the MOF such that an adsorption dependent change can be easily detected.

With this in mind, colloidal crystals (often referred to as 75 photonic arrays) composed of ordered arrays of sub- $\mu$ m oxide or polymer microspheres are capable of reflecting light of specific wavelength due to their periodic structure. This wavelength is sensitive to changes in the effective RI and/or lattice spacing of the crystal and hence photonic arrays have previously been 80 investigated as sensors. Hupp and co-workers recently employed a silica colloidal crystal as a signal transducer and coated this with a film of HKUST-1 to capture and concentrate specific analytes. ${ }^{99}$ As described earlier, the same group have previously employed MOF-Ag np arrays to measure changes in RI using ${ }_{85}$ LSPR spectroscopy, but here the particle array was not central to the technique rather a convenient way to prepare the device. ${ }^{54}$ 

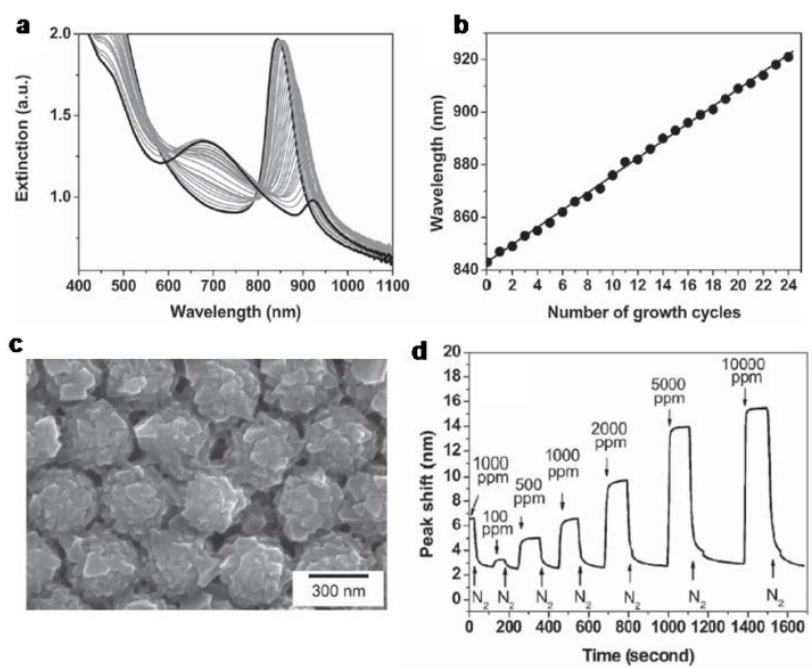

Fig. 28 (a) Visible and NIR extinction spectra of a HKUST-1 silica colloidal crystal composite. (b) Stop-band of the composite vs. the number of HKUST-1 growth cycles. (c) SEM image of the HKUST-1 5 silica colloidal crystal composite viewed from the top at high magnification. (d) Responses of the MOF-silica colloidal crystal to a series of $\mathrm{CS}_{2}$ vapours of various concentrations vs. time. (Adapted with permission from reference 99. Copyright 2011 Wiley-VCH)

A carboxylate-functionalised colloidal crystal thin film was 10 prepared from monodisperse silica microspheres and HKUST-1 was grown over this using the widely employed LbL method. After 24 growth cycles the array of silica microspheres maintained hexagonal order, and was covered homogeneously with intergrown 50-100 nm MOF crystals. Visible and NIR 15 extinction spectra revealed a linear red shift of the so-called stopband ( $\lambda$ of reflected light) with successive growth cycles consistent with a change in effective RI of the array with increasing MOF deposited. ${ }^{99}$ (Fig 28 a-c)

The photonic MOF composite arrays were activated under 20 high dynamic nitrogen flow to remove solvent and water molecules, which induced a blue shift of the stop band by a few nms. The sensor works as adsorption of guest molecules displace vacuum from the activated MOF, increasing the effective RI and inducing a red-shift of the stop-band. Indeed, upon exposure to

${ }_{25} \mathrm{CS}_{2}$ vapour a reversible red shift was rapidly observed, which increased with analyte concentration up to a maximum of $16 \mathrm{~nm}$ at 10,000 ppm of the analyte. (Fig. 28d) Water and EtOH vapours were also tested, and it is noted that while the size of the red shifts are different for each analyte at vapour concentrations 30 below 10,000 ppm these are similar at higher concentration. This indicates that the shifts are dependent on the amount of analytes adsorbed and their individual RIs.

From the observed shifts of the stop-band the authors calculated and normalised the volume fractions of analytes 35 adsorbed by the MOF and constructed adsorption curves, whose shapes and maximal uptakes agreed exceptionally well with those obtained for thin films of HKUST-1 by QCM. This is an important validation of the method and shows that adsorption isotherms are accessible from changes in effective RI if these can 40 be determined at a high enough resolution. Clearly the integration and stability of the photonic array is critical to the success of this method.

The energies of Fabry-Pérot interference peaks, observed when the thickness of a supported material or film is comparable to the 45 wavelength of light, are also sensitive to changes in RI. This property has been exploited by preparing transparent films of ZIF-8 supported on unmodified glass (or silicon) slides for selective sensing of gases and vapours. ${ }^{100}$ ZIF-8 films of initial 50 $\mathrm{nm}$ thickness were obtained by immersion of the slides into a 50 methanolic solution of the metal and ligand at room temperature, and thicker films could be prepared by repeating with fresh solutions. SEM images confirmed that the film thickness increases by $\sim 100 \mathrm{~nm}$ per growth cycle, and despite the MOF being colourless, the supported films displayed variable 55 colouration due to thickness-dependent optical interference effects in the visible region.
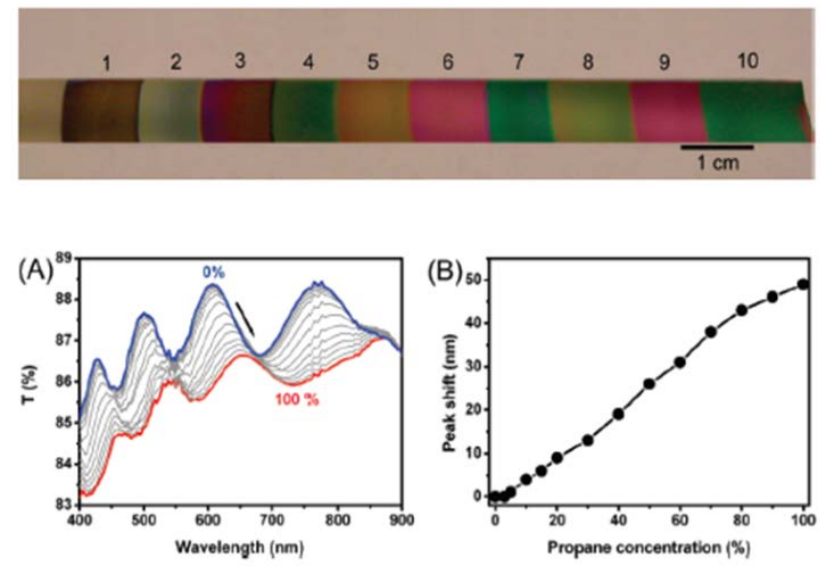

Fig. 29 Upper: Photograph of a series of ZIF-8 films of varying thickness grown on a silicon substrate. Lower: (A) UV-vis transmission spectra of a 60 10-cycle ZIF-8 film grown on glass after exposure to propane of various concentrations (blue curve for $0 \%$ and red curve for 100\%) and (B) corresponding interference peak (originally at $612 \mathrm{~nm}$ ) shift versus propane concentration. The propane concentration is expressed as a percentage of the total gas flow where $\mathrm{N}_{2}$ is used as diluent. (Adapted 65 with permission from reference 100. Copyright 2010 American Chemical Society)

Exposure of a $1000 \mathrm{~nm}$ thick ZIF-8 film to propane vapour shifts the Fabry-Pérot interference fringes arising from the transmission of visible light through the film by up to $49 \mathrm{~nm} .{ }^{100}$

70 This occurs rapidly and reversibly, and from the magnitudes of the shifts it is calculated that the volume fraction of propane in the framework is $\sim 0.13$. The sensor is responsive to a range of vapours and gases, and displays some selectivity based on molecular-sieving effects and framework hydrophobicity; for 75 example, $n$-hexane and ethanol are readily detected whereas bulky cyclohexane and polar water are not. Exposure to the vapour above EtOH/water mixtures of varying EtOH contents further yields a concentration-dependent response. Saturation occurs at $40 \% \mathrm{EtOH}$, which corresponds to the expected EtOH 80 uptake calculated from the single-crystal X-ray structure of ZIF8.

\section{MOFs at polymer interfaces}

Organic macromolecules have many desirable properties for the preparation of composite materials, including good mechanical, 85 thermal and chemical stability. They can be prepared from a vast range of monomeric species using a seemingly equally large 
number of techniques, to have diverse and abundant functionality and controlled molecular weight allowing tuning of their physical properties, shape and porosity. ${ }^{101}$ They are often solution processable, and in some cases these polymeric species can also 5 respond to external stimuli such as light, heat and $\mathrm{pH} .{ }^{102}$ Despite these obvious materials benefits, there remain relatively few MOF-polymer composites compared to their oxide counterparts although the number of reports is growing rapidly.

There are currently two types of MOF-polymer composite, 10 both of which will be reviewed here. The first involves growth of a MOF at a pre-formed polymer interface using direct synthesis or precursor solutions, and the second exploits the solution processability of the polymer to form a dispersion of MOF particles from which a MOF-polymer composite may be cast or 15 shaped through higher level processing.
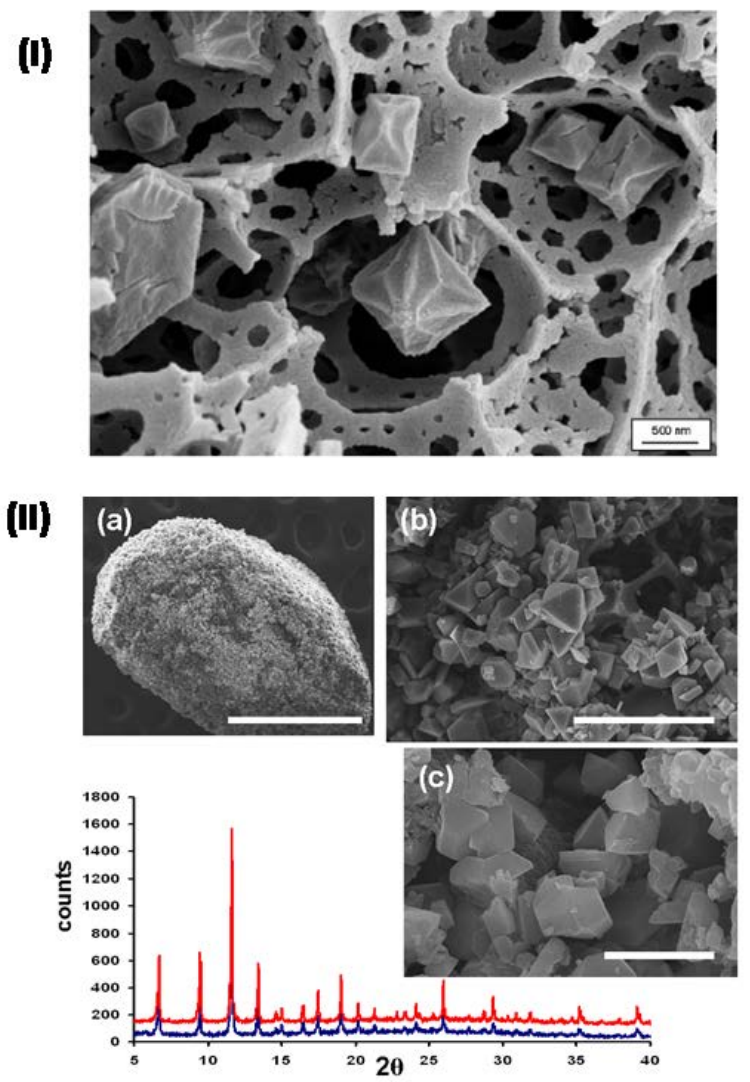

Fig. 30 (I) SEM image of the interior of a HKUST-1-polyHIPE composite after 3 impregnation cycles. (Reprinted with permission from reference 103. Copyright 2008 Wiley-VCH) (II) SEM and PXRD data of 20 a HKUST-1@PAM (PAM = polyacrylamide) composite prepared under solvothermal conditions. (a) SEM of a single composite bead; scale 1 $\mathrm{mm}$. (b) Cross section of a bead interior at a scale of $100 \mu \mathrm{m}$ and (c) 10 $\mu \mathrm{m}$. The X-ray patterns shown are for pure bulk HKUST-1 (red) and the HKUST-1@PAM composite (blue). (Reprinted with permission from 25 reference 31. Copyright 2010 Royal Society of Chemistry)

The first report of a MOF-polymer composite through deposition of a MOF on a pre-formed polymer support came from the group of Kaskel and co-workers. ${ }^{103}$ A macroporous polyHIPE monolith prepared from 4-vinylbenzyl chloride and 30 divinylbenzene was hydrophilised to promote MOF growth throughout the porous support. The polymer was impregnated with HKUST-1 precursor solutions, followed by solvothermal treatment to form the HKUST-1@polyHIPE composites. SEM images revealed MOF crystallites homogeneously distributed 35 within the interconnected macropores of the polymer scaffold (Fig 30, I), and a composition of MOF:polyHIPE of 3:2 was derived from TGA. The MOF loading level of the polyHIPE is tunable depending on the number of impregnation steps with the precursor solution, and the materials are stable during 40 hydrothermal MOF formation. All composites display type I adsorption isotherms consistent with the microporous nature of the MOF component, and the micropore volume correlates with MOF loading as expected.

Similar observations were reported by O'Neill et al for 45 HKUST-1 growth on macroporous mm-sized polyacrylamide (PAM) beads prepared using a sedimentation polymerisation technique. $^{31}$ (Fig. 30, II) Here the terminal amide groups expressed at the surface of the PAM were anticipated to coordinatively link the paddlewheel SBU of the MOF to the 50 polymer without the need for surface activation, and indeed high MOF loadings are observed in a one step process. It was noted that by pre-swelling the beads in solvent only (rather than a MOF precursor solution) prior to the MOF formation reaction, framework growth could be controllably restricted to the bead ${ }_{55}$ exterior through manipulation of the diffusion rate into the bead interior. A similar observation was also made for CPO-27, without pre-swelling of the beads, where the crystallites localised on the bead exterior were dominated by a smaller number of large crystals in contrast to the wide size distribution observed for 60 HKUST-1. The crystal size distribution is thus dependent on the detailed growth kinetics, and their location on how these kinetics compare to the diffusion rate of the framework-forming components into the bead interior.

While mechanical stirring of the HKUST-1@PAM composite 65 beads led to the erosion of some MOF crystallites from the bead exterior, SEM revealed that crystals within the PAM macropores did not change size or shape and are effectively protected by the flexible polymer support. Combine this with composite ease of handling, and it becomes possible to enhance the recovery and 70 recyclability of MOF active components from heterogeneous reaction systems. This is in contrast to bulk HKUST-1 tested under the same conditions, where well defined crystals experienced significant attrition and size reduction making it increasingly difficult to recover all of the material: this issue 75 would only be exacerbated with each successive (e.g. catalytic) cycle.

A further desirable property of the HKUST-1@polyHIPE and HKUST-1@PAM composites is the hierarchical nature of their porosity. ${ }^{31,103}$ Microporous materials tend to lack sufficient 80 transport pores which can limit the molecular flux of analytes or substrates for applications in separation or flow catalysis, respectively. However, by preparing microporous MOFs within the macropores of polymer supports this may be overcome since the resulting composites combine the excellent transport 85 properties of the macropores with the increased surface area and selectivity associated with micropores.

The macroporous polymers described above were configured as monoliths and beads, but pre-formed polymer membranes have also been employed as an interface for MOF growth. Yao et al 90 have used commercial solution permeable nylon membranes with 
a pore size of $0.1 \mu \mathrm{m}$ to prepare films of ZIF-8 on both sides of the substrate through a process they have termed contra-diffusion synthesis. ${ }^{104}$ Here the flexible macroporous membrane separates methanolic solutions of the metal and ligand in a diffusion cell, 5 and ZIF-8 crystallisation occurs when the reactants cross the membrane as shown schematically in Fig. 31. The polyamide functionality of the nylon makes this a suitable support for MOF growth. Crystal size and morphology is strongly dependent on the local concentrations of reactants with large crystals observed on 10 the metal side and nanocrystals on the ligand side, consistent with previous studies. Coverage of the support and thickness of the ZIF-8 films could be tuned by reaction time and the overall M:L ratio in the system, as well as repeated crystallisation methods.
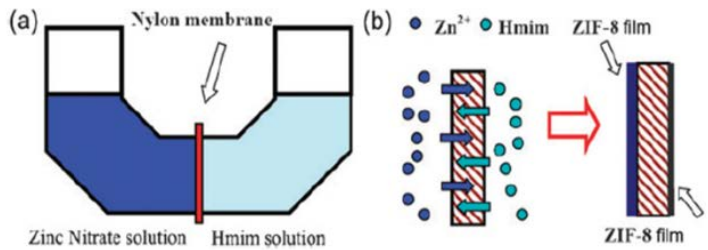

15 Fig. 31 (a) Diffusion cell for ZIF-8 film preparation and (b) the schematic formation of ZIF-8 films on both sides of the nylon support via contradiffusion of $\mathrm{Zn}^{2+}$ and Hmim (2-methylimidazole) through the pores of the nylon support. (Reprinted with permission from reference 104. Copyright 2011 Royal Society of Chemistry)

20 The quality of the ZIF-8@nylon membranes was investigated using gas permeation studies. $\mathrm{H}_{2}$ permeances decreased with reaction time indicating more complete coverage of the support, although at $10^{-6} \mathrm{molm}^{-2} \mathrm{~s}^{-1} \mathrm{~Pa}^{-1}$ these were higher than those reported for analogous ZIF-8 membranes supported on porous 25 oxides; perhaps indicative that by comparison the films prepared by contra-diffusion were less dense overall. This is reflected in their $\mathrm{H}_{2} / \mathrm{N}_{2}$ selectivities which only just exceed the Knudsen diffusion factor for this binary mixture for even the thickest membranes prepared.

30 As an alternative to the in-situ formation of MOF-polymer composites prepared by adding a preformed polymer to the MOF synthesis, Meilikhov et al have recently exploited the LbL growth strategy to prepare HKUST-1-polymer fibres. ${ }^{105}$ The relatively unreactive surface of polyester fibres were chemically modified 35 with polyvinylamine to increase the concentration of reactive groups, and these amines were subsequently transformed into carboxylate groups for efficient MOF binding by reaction with bromoacetic acid.

As previously outlined for MOFs deposited on SAMs on $\mathrm{Au}^{38}$, 40 the MOF-polymer fibres were built up by successive cycles of immersion of the functionalised (polymer) surface into metal and ligand solutions. Early stage MOF deposition during the first 10 cycles was monitored with UV-vis, where a linear increase in absorbance is indicative of a regular assembly process. ${ }^{105}$ (Fig. 45 32, upper) For untreated wool fibres however, a non-linear increase was observed likely arising from a disordered seed layer during the first few cycles that gradually became ordered with increasing deposition. After 40 cycles, sufficient material was deposited to examine the films with SEM (Fig. 32, lower) and

50 XRD which revealed, respectively, agglomerates of MOF crystallites on the polymer surface with no preferred orientation.
This is expected due to the rough nature of the polymer surface and tight packing of the fibres which prevent even coverage. As for other MOF-polymer composites, the MOF component of the 55 HKUST-1-polymer fibres retains its permanent porosity.
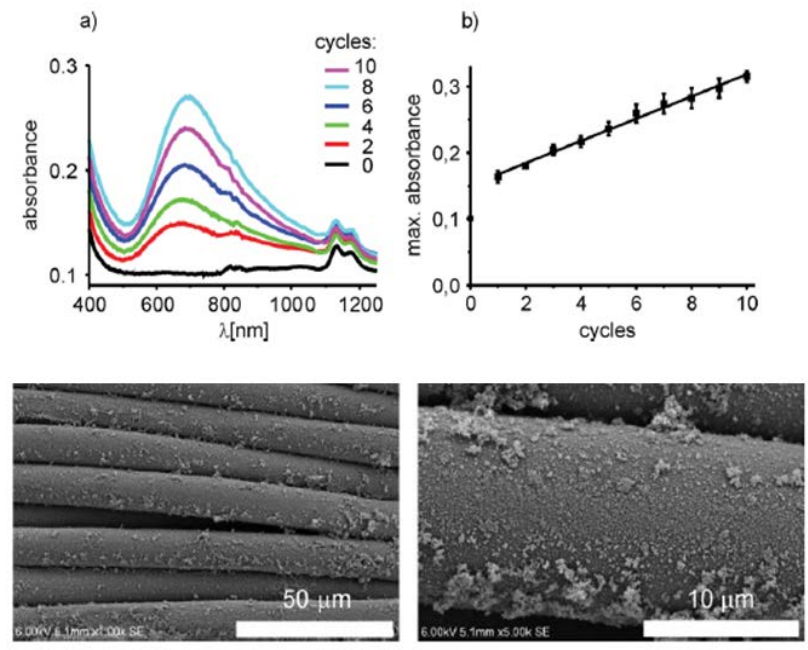

Fig. 32 Upper: (a) UV-vis of functionalised synthetic polyester fabric after with different numbers of layers of HKUST-1 and (b) the linear increase in absorbance at $704 \mathrm{~nm}$ as a function of the number of cycles. 60 Lower: SEM images of the MOF-polyester composite fibres after 40 growth cycles of HKUST-1. (Adapted with permission from reference 105. Copyright 2011 Royal Society of Chemistry)

LbL deposition has also been employed as a facile method to access photonic MOF-polymer hybrid films. Using a colloidal 65 crystal array of monodisperse $300 \mathrm{~nm}$ carboxylate-functionalised polystyrene (PS) spheres as a functional interface, Wu et al have prepared composite HKUST-1 films with an embedded photonic array that can provide the MOF with a sensitive optical signalling mechanism. ${ }^{106}$ SEM images reveal successful deposition of the ${ }_{70} \mathrm{MOF}$ onto the surface of the ordered PS array. The resulting linear red shift of the Bragg reflection of the colloidal array after each successive deposition cycle in the composites indicate uniform MOF growth which does not disrupt the array during the assembly process, and tunable optical properties. As outlined 75 earlier in this review, a similar HKUST-1-based sensing device using an analogous photonic silica array has been reported by Hupp and co-workers. ${ }^{99}$

The photonic MOF-PS composite films were activated and exposed to a range of solvent vapours, where the porous MOF 80 acts to capture guest molecules and the embedded array provides an optical readout. While the large pores of HKUST-1 were nonselective to the solvents tested, the position of the Bragg reflection was nevertheless shifted to varying degrees which appeared to follow a broad polarity trend: polar solvents blue 85 shift the peak whereas non-polar solvents cause a red shift. Given the structural stability and relative inflexibility of HKUST-1 compared to some other MOFs, it was concluded that changes in the effective RI rather than the MOF lattice per se upon guest adsorption was the most likely cause of the observed shifts. ${ }^{99,100}$

90 Many organic macromolecules are soluble in common solvents to form neutral polymer solutions or polyelectrolytes, gels and in the case of amphiphilic species such as block copolymers, micellar species or liquid crystal phases dominate. Whichever 
way the system behaves, this inherent solubility allows these macromolecules to be processed, which is advantageous for the preparation of application-specific materials configurations. One of the simplest things that can be realised is the casting of these 5 solutions into thin films to form polymer membranes for separation processes.

While some of the properties of polymer separation membranes are dependent on the exact conditions of casting, these wholly organic systems are typically characterised by low

10 permselectivity and poor chemical resistance compared to their inorganic (e.g. zeolite) counterparts. ${ }^{61}$ Conversely, inorganic membranes are not easily processable and tend to be rather brittle which can limit their utility. One solution to this juxtaposition is the mixed-matrix membranes (MMMs) ${ }^{107}$ that combine the 15 processability of organic polymers with the separating power and permeability of highly regular inorganic pores. There are many examples of zeolite-based MMMs, but there are clear advantages to preparing such systems from MOFs given their tunable pore size, diverse functionality and in some cases enantiopurity. The 20 interface between the inorganic adsorbent and the polymer is a key factor in producing MMMs of high selectivity. Adherence to the polymer support is often poor in the case of zeolite MMMs which hampers their use and reduces selectivity through the formation of non-selective voids at the polymer-zeolite 25 interface $^{108}$; however, the hybrid nature of MOFs is likely to enhance interactions via both the metal and ligand components with polymers bearing appropriate functionality. ${ }^{109}$

MOF-based MMMs are most often prepared by dispersing preformed MOF crystallites into a polymer solution prior to casting, 30 and several examples have now been reported for a variety of MOF and polymer combinations. Commonly used polymer phases for MOF-MMMs include the commercial polyimide Matrimid, polysulfone and poly(vinyl acetate) which all possess some polar functionality for interaction with the surface of MOF 35 crystallites.

Balkus Jr., Musselman and co-workers have successfully prepared and investigated the permselectivites of homogeneous MOF-MMMs of highly porous $\left[\mathrm{Cu}\left(4,4^{\prime}-\text {-bipy }\right)_{2}\left(\mathrm{SiF}_{6}\right)\right]$ and MOF-5 in Matrimid. ${ }^{110,111}$ The membranes were prepared by dispersing 40 MOF nanocrystals in a $10 \mathrm{wt} \%$ solution of Matrimid in chloroform, which was subject to extensive and repeated homogenisation cycles prior to casting on glass or Mylar substrates. Defect free MMMs with final MOF/polymer compositions of 10, 20 and $30 \mathrm{wt} \%$ were prepared in each case as 45 evidenced by SEM (Fig. 33, upper) and single-gas permeability data: higher MOF loadings however, result in particle aggregation leading to some defect formation. ${ }^{110}$ Mechanical strength tests of the $\left[\mathrm{Cu}\left(4,4^{\prime} \text {-bipy }\right)_{2}\left(\mathrm{SiF}_{6}\right)\right] /$ Matrimid $\mathrm{MMM}$ indicated increased rigidity compared to pure Matrimid and a 50 good adhesion between the polymer and the MOF particles. Some plastic deformation is observed in SEM images of MOF5/Matrimid MMMs, and this is also taken as evidence of strong MOF-polymer adhesion. ${ }^{111}$

Permeability for $\mathrm{H}_{2}, \mathrm{CO}_{2}, \mathrm{~N}_{2}, \mathrm{CH}_{4}$ and $\mathrm{O}_{2}$ through the ${ }_{55} \mathrm{MOF} / \mathrm{Matrimid} \mathrm{MMMs}$ was found to increase with MOF loading for both systems when compared to pure Matrimid; however, increases for MOF-5/Matrimid were significantly higher (up to $120 \%$ vs. Matrimid) than those observed for $\left[\mathrm{Cu}\left(4,4^{\prime}-\right.\right.$ bipy $\left.)_{2}\left(\mathrm{SiF}_{6}\right)\right] /$ Matrimid at the same loading level. This is further 60 borne out in the diffusivity of the (pure) gases calculated by the solution-diffusion model: gas diffusivities for MOF-5/Matrimid increase significantly with MOF loading whereas those for $\left[\mathrm{Cu}\left(4,4^{\prime} \text {-bipy }\right)_{2}\left(\mathrm{SiF}_{6}\right)\right] /$ Matrimid remain relatively constant. On the other hand, MOF-5/Matrimid MMMs do not show increased 65 solubility for any gas with increased loading whereas $\mathrm{CH}_{4}$ solubility in $\left[\mathrm{Cu}\left(4,4^{\prime} \text {-bipy }\right)_{2}\left(\mathrm{SiF}_{6}\right)\right] /$ Matrimid is increased 3-fold over the pure polymer at $40 \%$ loading where the other gases are not. (Fig. 33, lower)
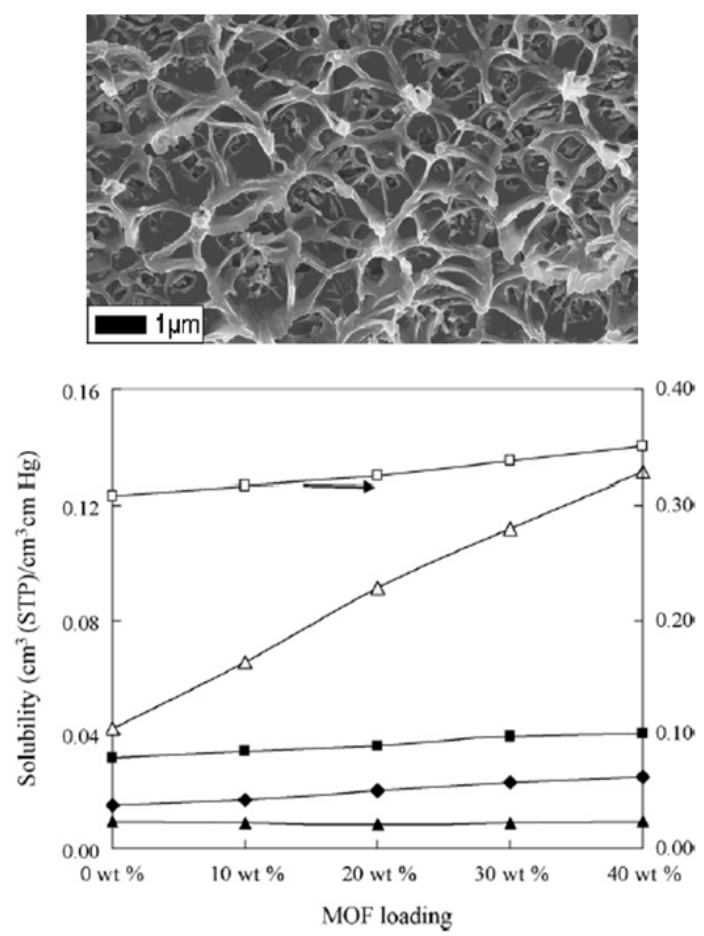

70 Fig. 33 Upper: SEM image of a cross-section of a $30 \%\left[\mathrm{Cu}\left(4,4^{\prime}-\right.\right.$ bipy $\left.)_{2}\left(\mathrm{SiF}_{6}\right)\right] /$ Matrimid mixed-matrix membrane. Lower: Solubility of gases in the MMM as a function of loading where $(\boldsymbol{\square}) \mathrm{O}_{2},(\square) \mathrm{CO}_{2},(\boldsymbol{\Delta})$ $\mathrm{H}_{2},(\diamond) \mathrm{N}_{2}$ and $(\Delta) \mathrm{CH}_{4}$. Note the increase in solubility for $\mathrm{CH}_{4}$ with loading whereas other gases remain relatively constant, indicating a 75 favourable affinity between this gas and the MOF. (Reprinted with permission from reference 110. Copyright 2008 Elsevier)

The above observations indicate that differences in pore structure, gas diffusion characteristics and affinities between the two MOFs are the dominant influence on MMM properties, 80 especially selectivity. For example, $\left[\mathrm{Cu}\left(4,4^{\prime} \text {-bipy }\right)_{2}\left(\mathrm{SiF}_{6}\right)\right]$ has a very strong affinity for $\mathrm{CH}_{4}$ permitting effective separation of $\mathrm{CH}_{4} / \mathrm{N}_{2}$ mixtures greater than the ideal selectivity using a $20 \%$ MOF-MMM. ${ }^{110}$ This arises from the increased solubility of $\mathrm{CH}_{4}$ due to the presence of the MOF. MOF-5/Matrimid MMMs were 85 found to be unselective for a particular gas, but 50:50 mixtures of $\mathrm{CO}_{2} / \mathrm{CH}_{4}$ and $\mathrm{N}_{2} / \mathrm{CH}_{4}$ displayed respective $\mathrm{CH}_{4}$ selectivity enhancements of 20 and $15 \%{ }^{111}$ In contrast to $\left[\mathrm{Cu}\left(4,4^{\prime}-\right.\right.$ bipy $\left.)_{2}\left(\mathrm{SiF}_{6}\right)\right] /$ Matrimid, this selectivity arises due to the increased solubility (hence retention) of $\mathrm{CO}_{2}$ and $\mathrm{N}_{2}$ in the polymer itself, 90 which facilitates (non-selective) $\mathrm{CH}_{4}$ transport through the pore structure of the included MOF-5 crystallites. What is abundantly clear from this is that both the MOF and polymer components of MMMs have an associated selectivity and permeability and these 
need to be matched carefully for high performance gas separations, which may be further facilitated using computational approaches. ${ }^{112}$

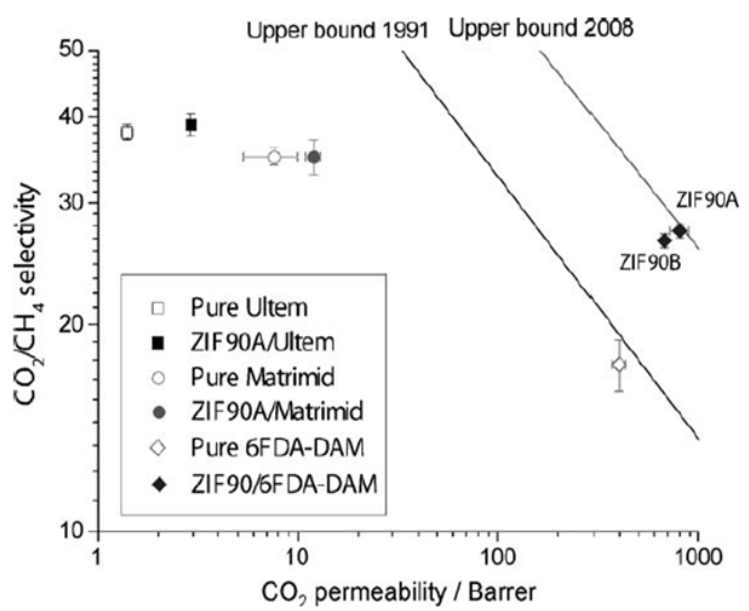

5 Fig. 34 Gas-permeation properties of mixed-matrix membranes containing $15 \mathrm{wt} \%$ of ZIF-90 crystals measured with pure gases. (ZIF90A and ZIF-90B refer to small differences in synthesis conditions of the MOF prior to membrane casting) Measurements were performed at $358^{\circ} \mathrm{C}$ and $4.5 \mathrm{~atm}$ upstream pressure for Ultem and Matrimid membranes, and 10 at $258^{\circ} \mathrm{C}$ and $2 \mathrm{~atm}$ upstream pressure for 6FDA-DAM membranes. The upper bounds for polymer membrane performance as defined in 1991 and 2008 are shown. (Reprinted with permission from reference 113. Copyright 2010 Wiley-VCH)

Matching of polymer and MOF properties to achieve superior 15 MMM performance has been elegantly demonstrated by Bae et al for ZIF-90/polyimide MMMs. ${ }^{113}$ Three polyimide matrices (Ultem, Matrimid and 6FDA-DAM) were employed to prepare MMMs loaded with 15 wt\% ZIF-90 microcrystals. As previously described ZIF-90 also contains additional aldehyde functionality, 20 which has the potential to further enhance interactions with the polymer matrices. For the Ultem and Matrimid systems, $\mathrm{CO}_{2}$ permeabilites were significantly increased relative to the pure polymers, while $\mathrm{CO}_{2} / \mathrm{CH}_{4}$ selectivity remained unchanged as shown in Fig. 34 arising from the permeability mismatch between 25 the two components according to Maxwell's model. However, 6FDA-DAM is a more highly permeable matrix than Ultem and Matrimid, and the resulting MMMs showed both enhanced permeability and selectivity over pure 6FDA-DAM due to greater compatibility between the two components. (Fig. 34) Indeed, the 30 ZIF-90/6FDA-DAM MMMs $\mathrm{CO}_{2}$ permeability was in excess of 700 Barrer with a $\mathrm{CO}_{2} / \mathrm{CH}_{4}$ selectivity of 37 under mixed gas conditions which exceeds the upper bound for polymer performance drawn in 1991. This indicates that small pore MOFs bearing extra-framework functional groups homogeneously 35 dispersed in polymer matrices of high permeability, are technologically extremely desirable.

MOF-based MMMs provide significant scope to optimise their performance for specific separations given the diversity of polymer matrices available, and the opportunity to select MOFs 40 of demonstrable potential for the desired application. For example, high $\mathrm{CO}_{2} / \mathrm{CH}_{4}$ selectivities have been reported for MMMs containing flexible frameworks that experience 'breathing' effects in response to guest loading. ${ }^{114}$ Bulk $\mathrm{NH}_{2}$ -
MIL-53(Al) exists in two forms depending on applied $\mathrm{CO}_{2}$ 45 pressure: a nanopore structure at low pressure and a more open expanded framework at higher pressures, leading to enhanced performance in $\mathrm{CO}_{2}$ capture over other MOFs.

Zornoza et al have prepared homogeneous MMMs by dispersing uniform microwave-assisted microcrystals of $\mathrm{NH}_{2}$ 50 MIL-53(Al) into a polysulfone (PSF) matrix up to $40 \mathrm{wt} \%$, permitted by specific interactions between the MOF and polymer. ${ }^{114}$ Single gas permeabilities through the MMM at 1 bar and $308 \mathrm{~K}$ increase slightly for $\mathrm{CO}_{2}$ and decrease for $\mathrm{CH}_{4}$ as a function of MOF loading, which contributes to the selectivity 55 trend under these low pressure conditions. Exceptional $\mathrm{CO}_{2} / \mathrm{CH}_{4}$ selectivities of 110 were recorded at higher pressures for MMMs containing $25 \mathrm{wt} \% \mathrm{NH}_{2}$-MIL-53(Al) as shown in Fig. 35 however, arising from the switch between the small and large pore forms of the MOF that typically occurs at 5 bar. It is further 60 speculated that the expansion of the crystals during this transition may serve to close defects at the MOF-polymer interface which would further improve membrane performance. This is an interesting prospect for MMM formation, as it might be expected that the inherent flexibility of some MOFs would be detrimental 65 to selectivity overall.

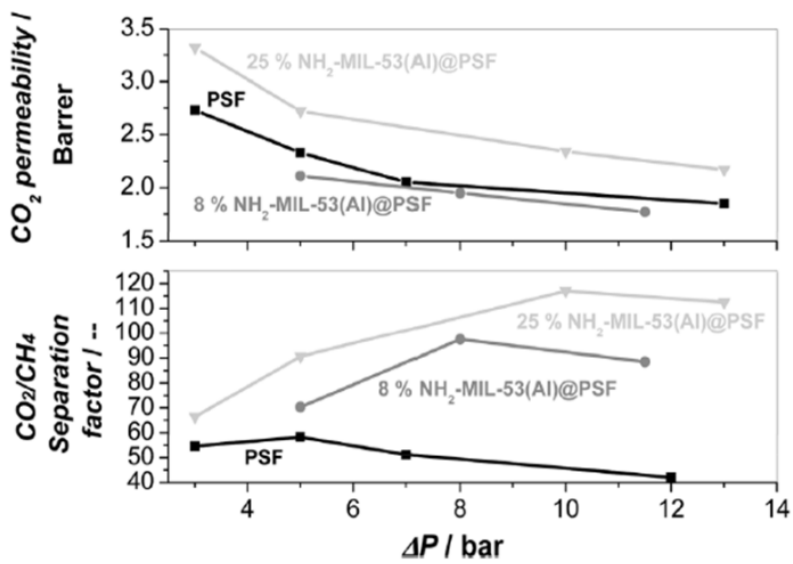

Fig. 35 Separation performance of different MIL-53(Al)-polysulphone mixed matrix membranes compared to that of the parent polymer for a mixture $\mathrm{CO}_{2}: \mathrm{CH}_{4}=1: 1$ as a function of the transmembrane pressure 70 difference at $263 \mathrm{~K}$ (pperm=1 bar). (Reprinted with permission from reference 114. Copyright 2011 Royal Society of Chemistry)

In addition to gas separation, MOF-MMMs have been employed in solvent resistant nanofiltration (SRNF) systems. ${ }^{115}$ SRNF typically involves the use of polymer membranes for non75 aqueous liquid phase separation, hence a major requirement for the polymer is high chemical stability in a wide range of organic solvents. For this reason, polydimethylsiloxane (PDMS), viz. silicone rubber, is usually the preferred matrix. Basu et al have prepared a number of PDMS-based MMMs incorporating a range 80 of stable MOFs with diverse pore characteristics, including HKUST-1, MIL-47, MIL-53(Al) and ZIF-8, and tested their efficacy for the separation of Rose Bengal (RB) from isopropanol. ${ }^{115}$

The MOF/PDMS MMMs were 30 - $35 \mu$ m thick and displayed 85 higher permeance, which increased with MOF loading, and less retention of RB compared to pure PDMS membranes. This is due to significant defect formation between the MOF and the support. 
However, if the surfaces of the MOF crystallites are modified with $N$-methyl- $N$-(trimethylsilyl) trifluoroacetamide (MSTFA), thus improving compatibility with the PDMS matrix, permeances are similar to those observed for the pure polymer membranes 5 and RB retention is significantly higher. This further highlights the importance of a robust interface between the two components in any MMM system.

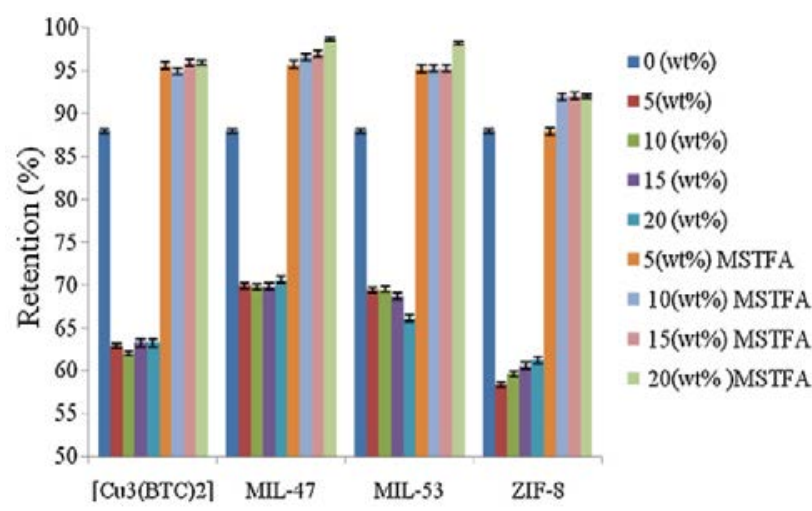

Fig. 36 Rose Bengal (RB) retention by a variety of MOF/PDMS solvent10 resistant nano-filtration membranes. Retention is less than the pure PDMS polymer in all cases, but can be significantly improved by modifying the surfaces of the MOF crystallites with $N$-methyl- $N$-(trimethylsilyl) trifluoroacetamide (MSTFA) to improve adhesion with the PDMS matrix and eliminating non-selective voids. (Reprinted with permission from 15 reference 115. Copyright 2009 Elsevier)

The effective separation of RB from isopropanol arises from the effect of the MOF on membrane swelling and the size exclusion effect of the microporous MOF itself. Membrane swelling is reduced in the presence of the MOF due to (physical) 20 cross-linking, which is even more pronounced in the MSTFA system. This reduces overall diffusivity of the larger RB, which also cannot enter the MOF pores resulting in greater retention.

ZIF-8/PMPS (polymethylphenylsiloxane) MMMs supported on a porous alumina capillary column have also been prepared, 25 and tested as organophilic pervaporation membranes for the separation of bio-alcohols from dilute aqueous solutions. ${ }^{116}$ The supported MMMs, synthesised using a solution-blending and dipcoating method, were $2.5 \mu \mathrm{m}$ thick and had an as-made composition of ZIF-8/PMPS of 1:10. The ZIF-8 nps were 30 distributed homogeneously throughout the PMPS matrix and, while EDXS mapping revealed a sharp transition between the alumina support and the ZIF-8, some intrusion of the silicone (PMPS) into the porous alumina was observed. This indicates a good adhesion between the MMM and underlying support and is 35 clearly beneficial in terms of overall composite stability.

The ZIF-8/PMPS membranes have a high permeance of 6000 7000 GPU (GPU $=1 \times 10^{-6} \mathrm{~cm}^{3}(\mathrm{STP}) \mathrm{cm}^{-2} \mathrm{~s}^{-1} \mathrm{cmHg}^{-1}$ ) for isobutanol, and the relative hydrophobicity of the MOF permits efficient recovery of the alcohol from $1-3 \mathrm{wt} \%$ aqueous 40 solutions, concentrating the isobutanol content of the permeate by up to 30 times. ${ }^{116}$ Isobutanol permenace and selectivity increase with increasing ZIF-8 content, attributed to an increased number of favourable pathways resulting from its high adsorption selectivity for isobutanol. Selectivity for other (linear) alcohols 45 over water was also increased for the ZIF-8/PMPS MMM over pure PMPS systems. Performance improved with increasing number of carbon atoms of the permeant, peaking at a separation factor of $\sim 38$ for $n$-butanol; a slight decrease was observed for $n$ pentanol due to the close interplay of adsorption and diffusion 50 effects.

This impressive performance, which was compared to a silicalite-1/PMPS reference membrane, corresponds to a comparatively low membrane area required for the separation of isobutanol, making this an economically feasible alternative to 55 energetically expensive distillation procedures.
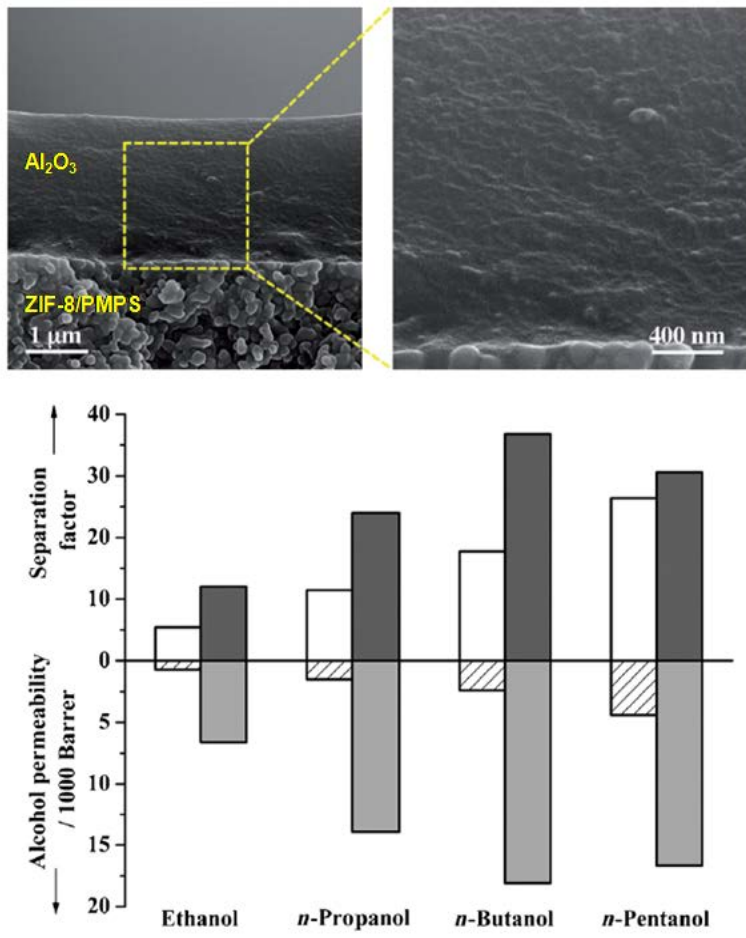

Fig. 37 Upper: Cross-section SEM images of a ZIF-8/PMPS mixedmatrix membrane supported on a porous alumina capillary column. Lower: Separation factor and alcohol permeability of PMPS (open and 60 line filled columns) and ZIF-8-PMPS (gray and light gray columns) membranes for aqueous solutions of C2-C5 alcohols (1.0 wt\% alcohols, $80^{\circ} \mathrm{C}$ ). 1 Barrer $=1 \times 10^{-10} \mathrm{~cm}^{3}$ (STP) $\mathrm{cm} \mathrm{cm}^{-2} \mathrm{~s}^{-1} \mathrm{cmHg}^{-1}$. (Adapted with permission from reference 116. Copyright 2011 Wiley-VCH)

Related to the MOF-based MMMs and SRNF systems ${ }_{65}$ described above, Ananthoji et al report the preparation and controlled drug release of MOF-hydrogel composites. ${ }^{117}$ In contrast to the MMMs, the MOF is dispersed in a solution of functional monomers and cross-linkers (rather than a soluble polymer) which is subject to UV-initiated polymerisation to form 70 the desired MOF-hydrogel composites. The MOF employed in this study is a Na-exchanged ZMOF of composition $\left[\mathrm{Na}_{48}\right]\left[\mathrm{In}_{48}(\mathrm{HImDC})_{96}\right] \quad$ (ImDC $=4,5$-imidazoledicarboxylate) which is characterised by its large pore size $(1.8 \mathrm{~nm})$ and potential in cation exchange, including pharmaceutically active 75 compounds such as anti-arrhythmic drug Procainamide hydrochloride ( $\mathrm{PHCl})$.

A series of large pore poly(HEMA/DHPMA/VP/EGDMA) hydrogels and ZMOF-hydrogel composites of varying VP (Nvinyl-2-pyrrolidone) content (required for porosity) were 80 prepared, which all displayed degrees of water adsorption in the range required for practical drug delivery. Further, the ZMOF 
crystallites afforded additional stability to the hydrogels through surface interactions with the gel matrix as evidenced by elevated glass transition temperatures. Optimum stability was observed for a $\mathrm{PH}$-loaded $(\mathrm{PH}=$ protonated procainamide) $\mathrm{ZMOF}$ in a VP30 5 (30 wt\% VP) hydrogel, which was studied for cation-induced $\mathrm{PH}$ release. Drug release is initially rapid from both the ZMOFhydrogel composite and a VP30 hydrogel control, followed by a slower more sustained release profile. However, delivery from the composite is slower at all stages of release, due to 10 electrostatic interactions between the charged framework and the $\mathrm{PH}$. While notable that the framework remains intact throughout this process, ZMOF analogues prepared from biologically benign metal ions rather than In(III) will be necessary for future applications. ${ }^{17}$
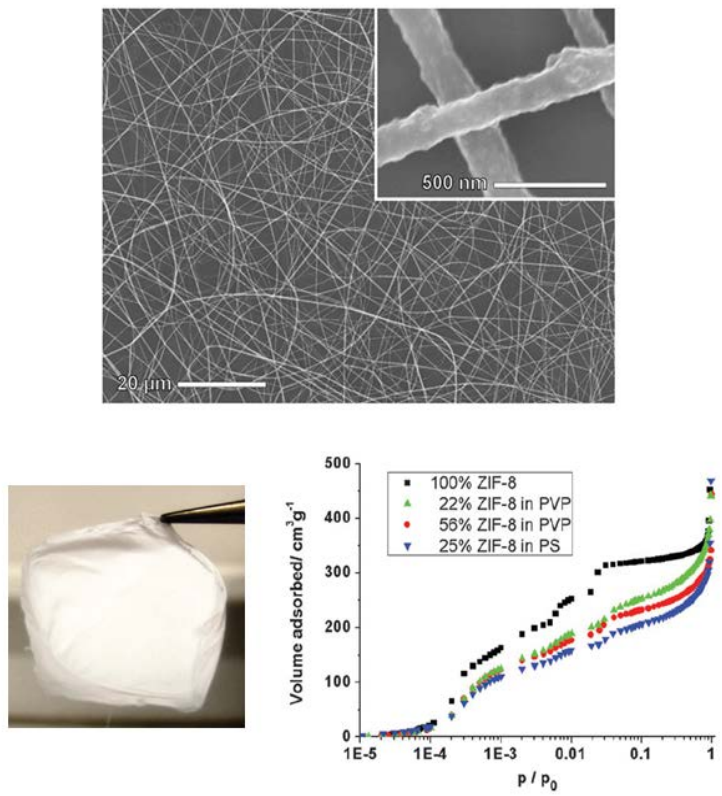

Fig. 38 Upper: SEM image of ZIF-8 nps in PVP fibres formed by electrospinning. The digital photograph shows a non-woven ZIF-8/PVP fibre mat. The $\mathrm{N}_{2}$ adsorption isotherms clearly demonstrate the accessible porosity of the ZIF-8 nps in both PVP and polystyrene (PS) matrices. 20 (Adapted with permission from reference 118. Copyright 2011 Royal Society of Chemistry)

For a level of processing greater than simple membrane casting, the technique of electrospinning has recently been employed for the preparation of porous MOF-polymer composite 25 nanofibres and textiles with potential application in personal protective systems. ${ }^{118,119}$ Electrospinning is primarily used for the preparation of thin uniform polymer fibres (both solid and hollow) as an electrified jet of polymer solution is rapidly and continuously stretched due to electrostatic repulsions between 30 surface charges and the evaporation of solvent. ${ }^{120}$ By including inorganic precursors or particles, metal salts, carbon nanotubes and porous zeolites in the polymer solutions, fibrous oxides and composites of diverse composition and enhanced properties have been prepared.

35 Most recently, Ostermann et al reported electrospun nanofibres of ZIF-8/PVP (PVP = polyvinylpyrrolidone) with diameters in the range 150-300 $\mathrm{nm}$, and their macroscopic non-woven fibre mats. $^{118}$ (Fig. 38) MOF loading levels of up to $50 \%$ were achieved, and SEM revealed the MOF nps were homogeneously ${ }_{40}$ dispersed within the polymer fibres. This is in contrast to the work of Rose et al who reported MOF-polymer composites of pearl necklace or spider web morphologies as shown in Fig. 39, where larger microcrystals of HKUST-1 or MIL-101 were stuck to or embedded within networks of pure polymer nanofibres up to ${ }_{45} 80 \%$ MOF loading. ${ }^{119}$ This demonstrates the importance of particle size in determining the morphology of the electrospun composites, and suggests nps are best if distribution of the MOF within, rather than between, the polymer fibres is an application requirement. $\mathrm{N}_{2}$ adsorption isotherms of both the ZIF-8/PVP (Fig. 5038 ) and microcrystal-polymer composites revealed MOF porosity remained fully accessible; however, for ZIF-8/PVP a reduction in adsorption kinetics over pure ZIF-8 nps was recorded as a result of the polymer coating.
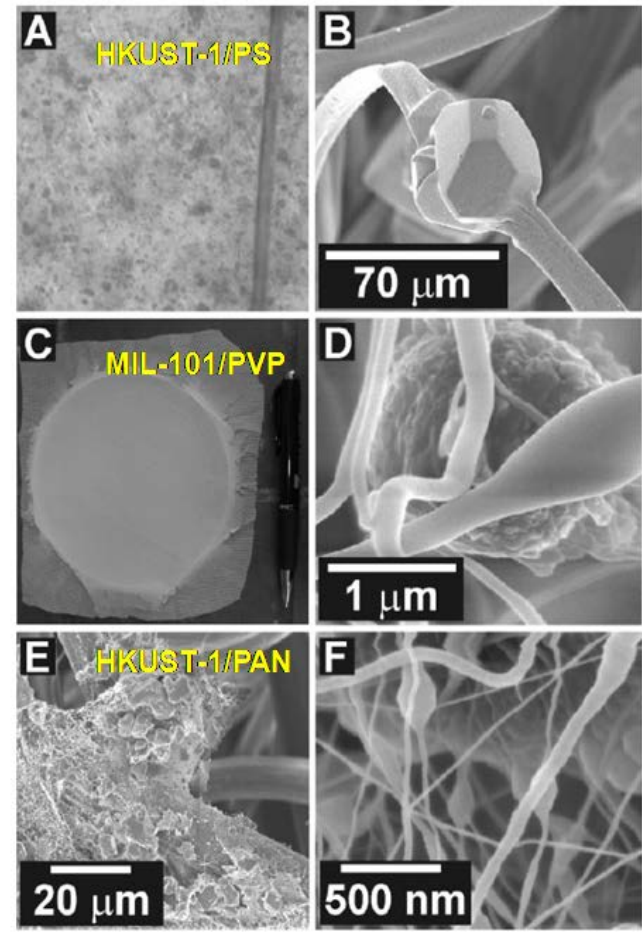

55 Fig. 39 Images (A, C) and SEM micrographs (B, D, E, F) from electrospun MOF/fibre composites: (A) HKUST-1/PS fibres in relation to a human hair, (B) HKUST-1 crystal on a PS fibre analogous to a pearl necklace, (C) homogeneous non-woven MIL-100(Fe)/PVP layer on polypropylene, (D) MIL-100(Fe) particle in PVP fibre web, (E, F) 60 HKUST-1 particles (BASF) in PAN fibres on a PAN non-woven (large substrate fibres in background). (PS = polystyrene; $\mathrm{PAN}=$ polyacrylonitrile). (Reproduced from ref 119, permission sought) (Reprinted with permission from reference 119. Copyright 2011 Wiley$\mathrm{VCH})$

65 Accessibility to the porosity and hence functionality of the MOFs is clearly important for future applications of these materials, and in the case of MOF nps is strongly dependent on the thickness and nature of the polymer, permitting tuning of adsorption kinetics. ${ }^{118}$ Chemical stability of the nanofibres is also 70 dependent on the nature of the polymer; for example, electrospun MOF-polymer composites prepared from PVP are water soluble whereas those derived from polystyrene (PS) or polyacrylonitrile (PAN) are not. By combining electrospinning of polymer solutions with the structural and functional diversity of MOFs 
and the increasing number of methods by which to control their particle size $\mathrm{e}^{74,121}$, there exists tremendous scope to prepare application-specific MOF-polymer composite fibres and textiles. For example, the recent demonstration that MOFs can 5 competitively adsorb large quantities of harmful organics such as models for the chemical warfare agents Sarin and mustard gas in the presence of moisture ${ }^{122}$, suggests electrospinning into textiles could lead to a new generation of militarily important MOFbased personnel protective systems.

\section{${ }_{10}$ MOFs at carbon interfaces}

Carbon-based materials such as carbon nanotubes (CNTs), graphite and graphene are of exceptional technological interest for their mechanical strength, hydrophobicity, potential in adsorption and catalysis, and interesting electronic properties. ${ }^{123}$ 15 It is therefore no surprise that they have been widely employed in composite systems for diverse applications, ranging from energy storage and delivery to fillers in matrices for biomedical devices and other lightweight but strong building materials. ${ }^{124}$

Oxidation of graphite increases its hydrophilicity through the 20 introduction of differential surface functionalities and defects, such that the resulting graphite oxide (GO) constitutes a water dispersible functional carbonaceous interface for enhanced interactions with small molecules. Bandosz and co-workers exploited the facile delamination and functional nature of the GO 25 surface for composite preparation, and have reported the synthesis and ammonia adsorption properties of a number of MOF-GO nanocomposites including MOF-5 ${ }^{125,126}$,HKUST-1 ${ }^{127}$ and MIL-100(Fe) ${ }^{128}$, by simply dispersing GO powder (up to 50 wt\%) in the usual MOF synthesis.

30 MOF-5 and HKUST-1 crystallites interact strongly with the hydroxyl, epoxy and carboxylate groups expressed at the GO surface to readily form MOF/GO composite materials as shown by PXRD, FTIR and adsorption studies. ${ }^{126,129}$ It is proposed that the MOF-5/GO composites are comprised of a sandwich like 35 structure of alternating MOF/GO/MOF layers, although the SEM images shown in Fig. 40 indicate composite morphology changes as GO content increases; perhaps through preferential MOF interaction with the carboxylate groups expressed mostly at the edges of the GO sheets. ${ }^{125}$ Composite formation with 40 carbonaceous material is dependent on the functional groups at the GO surface - a related study with HKUST-1 and unfunctionalised graphite reveals only the formation of physical mixtures $^{130}$ - and the relative orientation of metal coordination sites on the MOF available for GO binding as highlighted in Fig. ${ }_{45} 41{ }^{128}$ For example, MIL-100(Fe) forms disordered MOF/GO composite materials, arising from interaction of its spherical cages with GO layers rather than the cubic MOF-5 and HKUST-1 structures, where metal coordination sites lie along regular planes. (Fig. 41)
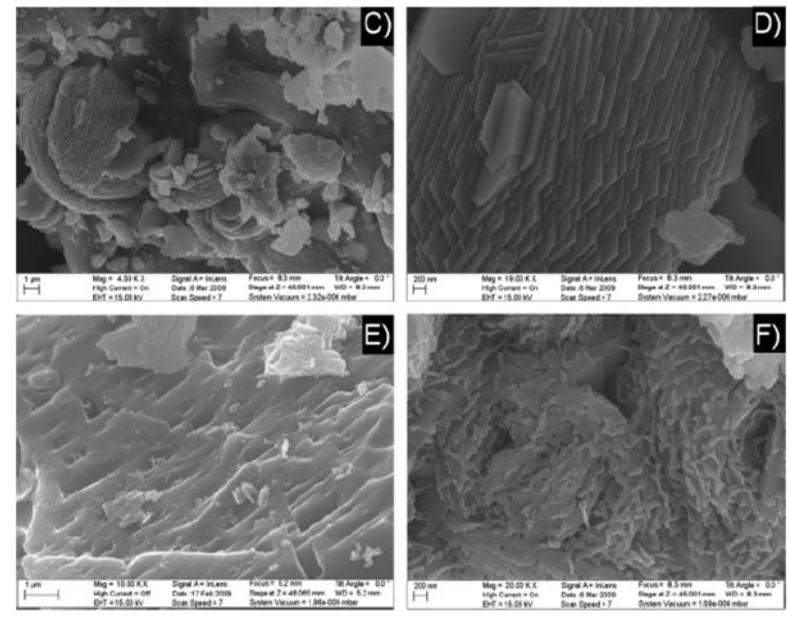

50

Fig. 40 SEM images for MOF-5/GO composites at GO loadings of (C) 5 wt \%, (D) higher-magnification of $5 \mathrm{wt} \%$ sample, (E) $10 \mathrm{wt} \%$ and (F) 20 $w t \%$. A clear change in morphology from the layer-like structures observed at 5 and $10 \mathrm{wt} \%$ of GO is seen on increasing the carbon content. 55 (Reprinted with permission from reference 125. Copyright 2011 Wiley$\mathrm{VCH})$

Ammonia adsorption by the MOF/GO composites is strongly dependent on the porosity and chemical nature of the MOF, and the nature and synergy of the MOF/GO interface, shown 60 schematically in Fig. 42 for HKUST-1/GO composites. Both MOF-5/GO and HKUST-1/GO composites display increasing ammonia adsorption capacity and retention with increasing GO content at ambient temperature, which generally exceed the expected capacity for a simple physical mixture of the same 65 composition; however, very high GO loadings tend to have a negative effect on performance. ${ }^{126,127}$ This increased capacity is primarily attributed to a synergetic effect between the two components where the more favourable surface of GO and small pore spaces arising at the interface between the GO and MOF 70 (Fig. 42) act cooperatively to enhance both ammonia and hydrogen physisorption through increased dispersion forces. ${ }^{129}$ By contrast, the poor interface between MIL-100(Fe) and GO (Fig. 41) leads to a decrease in adsorption capacity with GO content. $^{128}$

75 The specific nature of the MOF also plays a role in the adsorption process, particularly where there can be strong chemical interactions between $\mathrm{NH}_{3}$ and the MOF. MOF-5 is moisture sensitive and due to its very low ammonia uptake arising from the large pore structure and relatively weak 80 physisorptive interactions, it is the nature of the interface which is vital to enhanced adsorption. On the other hand, HKUST-1 and MIL-100(Fe) contain additional reactive sites for adsorption to occur. Activated HKUST-1 for example has open metal sites arising from removal of water molecules bound to the ${ }_{85}$ paddlewheel SBUs; these are known to coordinate a variety of molecules, and bind readily to ammonia and $\mathrm{H}_{2} \mathrm{~S}$ in the HKUST1/GO composites. ${ }^{127,131} \mathrm{NH}_{3}$ and $\mathrm{H}_{2} \mathrm{~S}$ adsorption by the HKUST1/GO composites follow the same multi-step adsorption behaviour: increased adsorption occurs due to the MOF/GO 90 interface and reactive binding to the $\mathrm{Cu}$ sites (Fig. 42), which ultimately leads to an irreversible reaction between the MOF and the adsorbate. This necessarily makes it difficult to understand the exact role of the GO in complex adsorption systems. 


\section{Cite this: DOI: 10.1039/c0xx00000x}
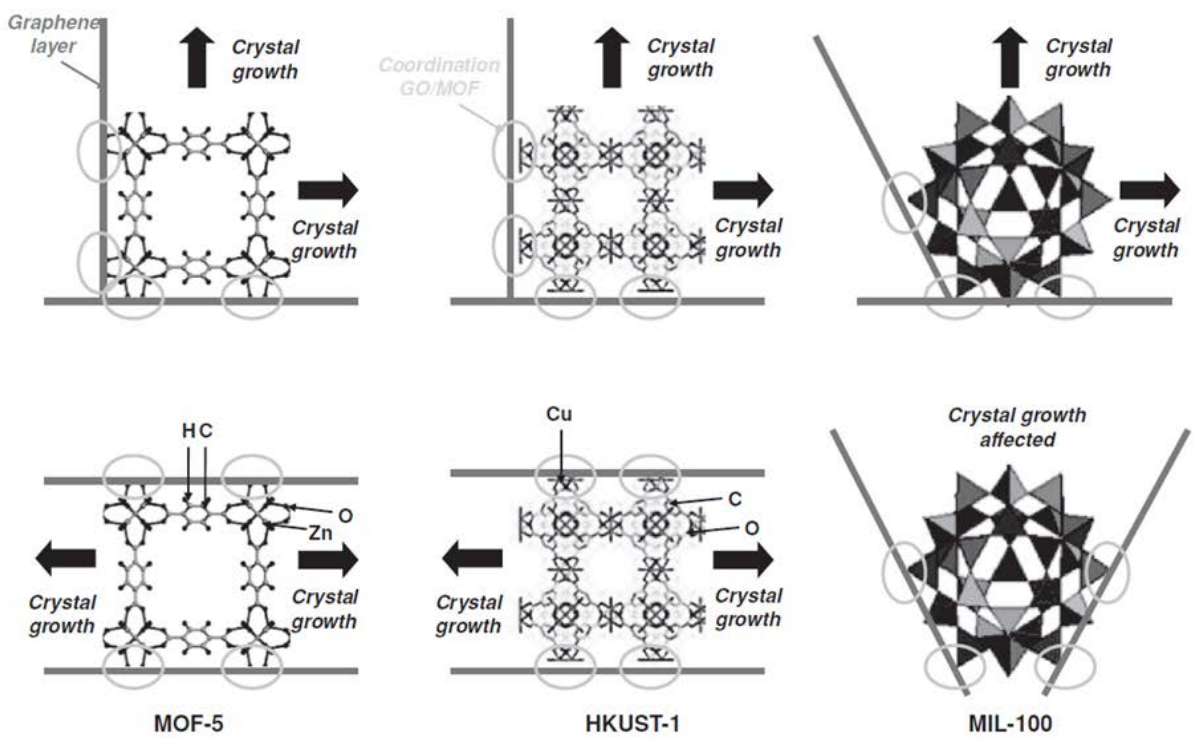

Fig. 41 Schematic comparison of the coordination between GO carbon layers to the MOF units for different types of MOF network: MOF-5, HKUST-1 and MIL-100(Fe). For MOF-5 and HKUST-1: atoms involved in coordination are indicated. For MIL-100(Fe): the red pyramids represent supertetrahedra units made of trimers of iron octahedra linked by molecules of BTC. (Reprinted with permission from reference $128 . \mathrm{Copyright} 2011 \mathrm{Wiley-VCH}$ )

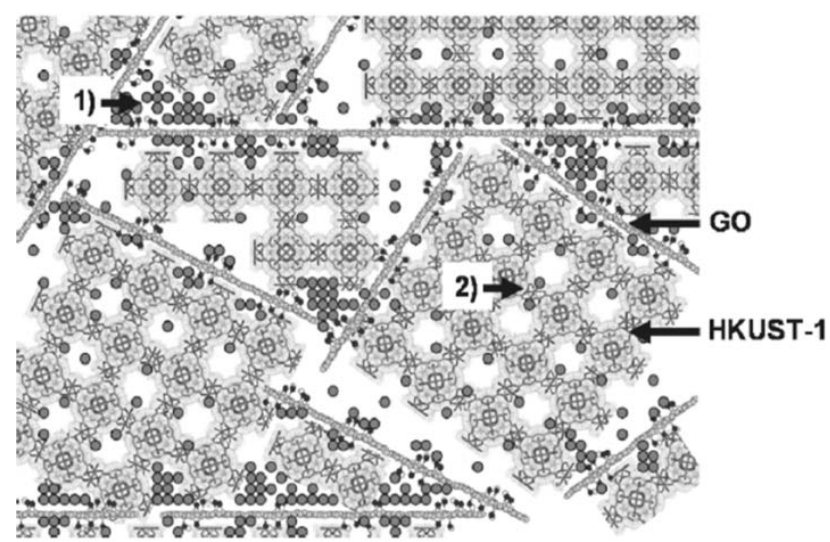

Fig. 42 Simple visualization of the two sites of ammonia adsorption in HKUST-1/GO composites with (1) physisorption at the interface between graphene layers and MOF units and (2) binding to the copper centers of 10 the paddlewheel SBUs. Ammonia molecules are represented by the dark gray circles. Note that the relative MOF and GO domain sizes are not to scale. (Reprinted with permission from reference 127. Copyright 2010 American Chemical Society)

Unlike MOF-5/GO, ammonia adsorption in HKUST-1/GO 15 composites is enhanced in humid conditions due to dissolution of the adsorbate, and moist conditions also appear to slow down reaction with the MOF despite an increased $\mathrm{NH}_{3}$ concentration, likely resulting from competitive water binding. For MIL100(Fe)/GO composites ammonia is also retained by interaction 20 with open metal sites, and Brønsted interactions with water molecules are also involved.
GO itself has a relatively low density of carboxylate groups on the surface of the sheets; rather, this functionality tends to be most prevalent on the sheet edges. This has some consequences 25 for MOF growth as seen in the work of Petit et al, where MOF5/GO composites tend to form layered materials at low GO content but disordered wormlike structures at high GO loading due to the greater number of carboxylate interactions with increasing GO favouring edge growth. ${ }^{125}$ This does suggest a 30 possible role of GO as a structure-directing agent for MOF growth, especially if the density of carboxylate functionalities could be increased, making this more akin to a SAM. Jahan et al reacted reduced GO with a phenyl carboxylic diazonium salt to prepare benzoic-acid functionalised graphene (BFG) that has a 335 fold increase in carboxylate functionality over GO, and subsequently used this as a functional interface for MOF growth. $^{132}$

MOF-5/BFG composites were prepared with BFG loadings of 1,4 and 5 wt $\%$. At the 1 wt\% level, a typical sandwich type 40 structure as reported for GO is observed, however BFG induces greater distortion of the MOF-5 lattice presumably arising from the greater interaction between its higher surface density of carboxylate groups and the MOF-5 SBUs. More significantly, MOF-5/BFG-5wt\% composites display a uniform nanowire 45 morphology $^{132}$ (Fig. 43) that is not observed for MOF-5/GO hybrids. The MOF-5/BFG nanowires are $300 \mathrm{~nm}$ in diameter and are highly oriented along the [200] direction, consistent with MOF-5 growth on carboxylate-terminated SAMs on Au. ${ }^{39} \mathrm{~A}$ kinetically controlled template growth model is proposed for the 50 composites based on Micro-Raman spectroscopy and mapping (Fig. 43) and AFM data which indicate that all BFG sheets are 
intercalated within the structure, and have a diameter close to that of the nanowires themselves.

Nitrogen adsorption properties of the MOF/BFG nanowires are comparable to that of the bulk MOF, indicating the composite 5 retains open porosity. The electrical properties of single composite nanowires were investigated, and while these were insulating at low field, current increased significantly at voltages $>4 \mathrm{~V}$ due to thermionic emission as shown by the $I-V$ curves in Fig 44. This can be further enhanced under white-light 10 illumination due to photoinduced charge transfer. ${ }^{132}$ Similar observations are recorded for a dye-loaded MOF/BFG nanowire, which permit photoexcitation and subsequent charge-injection by the included guest producing a significant enhancement in the photocurrent. (Fig. 44) This could open up the possibility of MOF 15 composite photocatalysis if appropriate electrical properties can be imparted onto otherwise insulating frameworks.
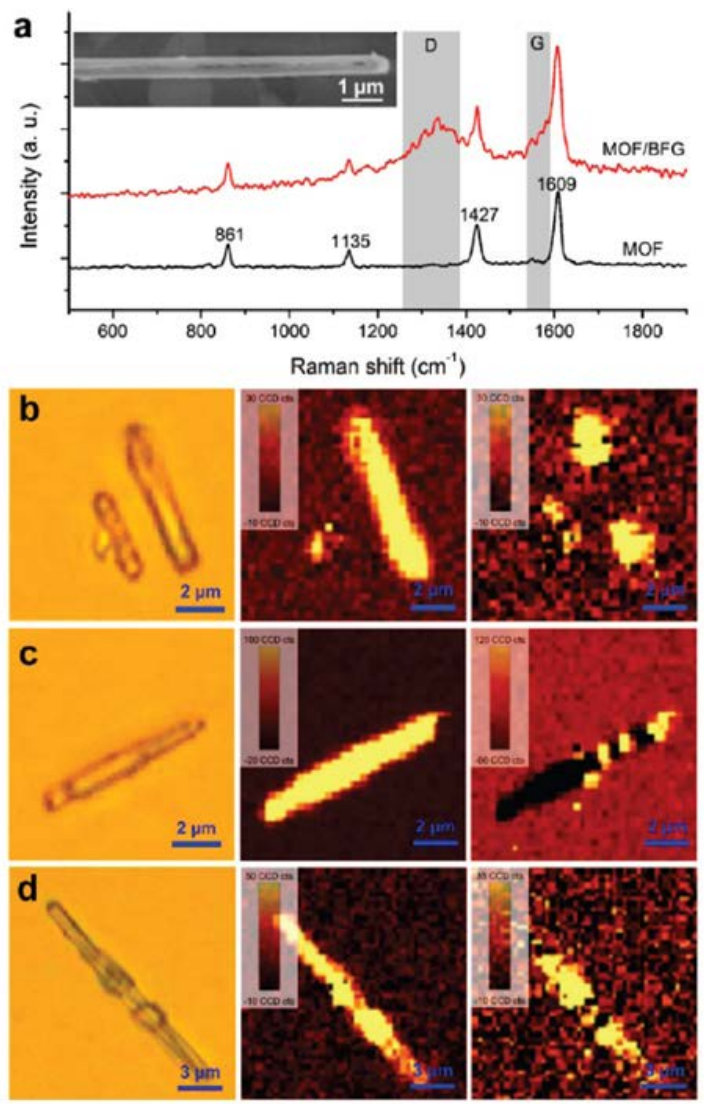

Fig. 43 (a) Raman spectra of MOF and MOF/BFG (5 wt \%) composites with graphene $G$ and $D$ bands highlighted. Insert shows SEM image of an 20 individual MOF/BFG nanowire. (b-d) Raman mapping of MOF/BFG nanowires. Left: optical image. Middle: Raman maps integrated by MOF Raman band at $1609 \mathrm{~cm}^{-1}$. Right: Raman maps integrated by D band of graphene which can be used to unambiguously locate graphene in the nanowires. (Reprinted with permission from reference 132. Copyright 252011 American Chemical Society)

CNTs have also been used as surfaces for MOF growth, particularly for the preparation of composite materials with enhanced gas storage capacity. Yang et al have reported the hydrogen storage properties of MOF-5/CNT composites $^{133}$, 30 including in the presence of Pt nps. ${ }^{134}$ The composites were prepared by adding acid-treated multi-walled CNTs (MWCNTs) or Pt-loaded MWCNTs dispersed in DMF to the MOF-5 synthesis mixture. As is the case for GO, acid treatment of the MWCNT surface prior to composite formation introduces 35 carboxylate functionalities for MOF binding. HRTEM and selected area diffraction of the MOF-5/CNT composites clearly demonstrate efficient admixing of the two components (Fig. 45), accounting for their enhanced thermal and moisture stability and higher Langmuir surface area than MOF-5 alone. The higher 40 surface area likely arises from interfacial ultramicropores (cf. MOF-5/GO) and the increased thermal stability (of the composite) permitting more efficient solvent removal during activation. ${ }^{133}$ A recent report by Chen et al details the increased moisture and electron beam stability of MOF-5 confined within 45 the interior of MWCNTs, where stability is dependent on the number of walls. ${ }^{135}$ It is not clear however, why the MOF-5 is restricted to the CNT interior in this case, as the reported synthesis appears identical to that of Yang et al. ${ }^{133}$
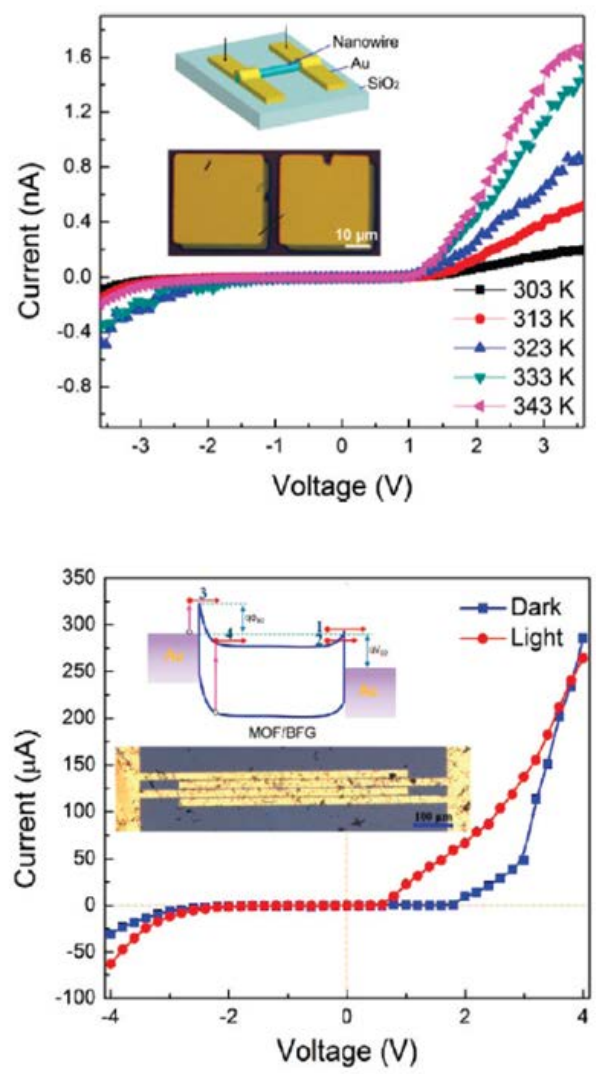

50 Fig. 44 (Upper curves) $I-V$ curve of single MOF/BFG nanowire measured at different temperatures. Insert shows schematic (upper) and optical image (lower) of the two-electrode device. (Lower curves) $I-V$ curve and photocurrent measured on an interdigitated device using MOF/BFG nanowires adsorbed with dye molecules. Lower insert shows the optical 55 image of the device. Upper insert illustrates the schematic energy band diagram of the device. 1. Thermionic emission; 2. Tunneling; 3. Excitation over the barrier; 4. Band-to-band excitation. (Adapted with permission from reference 132. Copyright 2011 American Chemical Society)

60 Hydrogen storage capacity of MOF-5/CNT at 1 bar and $77 \mathrm{~K}$, increased by $25 \%$ compared to the parent MOF as shown by the isotherms presented in Fig. 46, which is further enhanced at 
higher pressures where a 100\% increase is observed (298 K, 95 bar). This increased $\mathrm{H}_{2}$ capacity under a wide range of experimental conditions is largely attributed to the increased porosity at the interface and the improved structural integrity of 5 the MOF component. Similar results were reported for composites prepared from Pt-loaded MWCNTs, where $\mathrm{H}_{2}$ uptake increased by $50 \%$ over the pure MOF under cryogenic conditions and a $4.2 \mathrm{x}$ enhancement was observed under higher pressures, suggesting $\mathrm{MOF} / \mathrm{CNT}(\mathrm{Pt})$ composites may have utility as ${ }_{10}$ practical hydrogen spillover systems. ${ }^{136}$

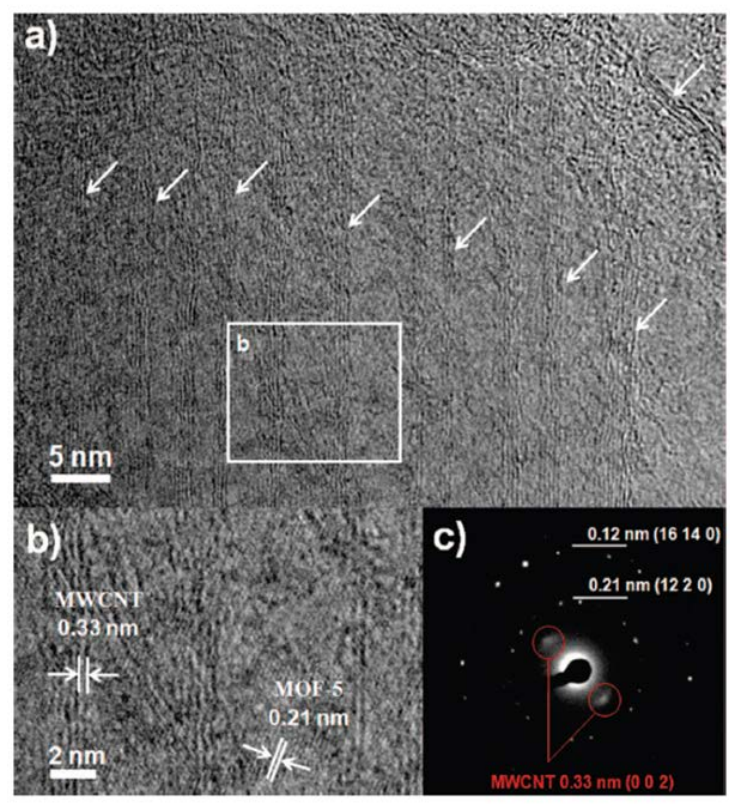

Fig. 45 HRTEM micrograph of a MOF-5/CNT composite crystal (white arrows indicate MWCNT lattices). (b) Enlarged view of the boxed area in (a), and (c) typical selected area electron diffraction patterns of (a). 15 (Reprinted with permission from reference 133. Copyright 2009 American Chemical Society)

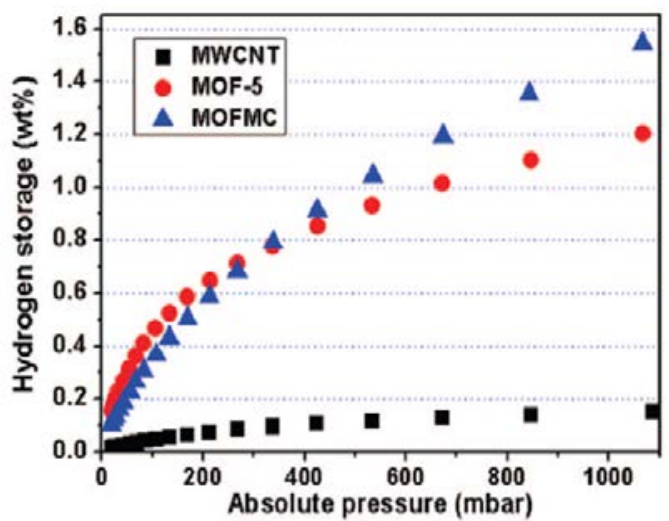

Fig. $46 \mathrm{H}_{2}$ adsorption isotherms measured at $77 \mathrm{~K}$ and 1 bar using a volumetric method for MOF-5/CNT composites (labelled MOFMC in the 20 figure legend) compared to the parent MOF and bare MWCNTs. (Adapted with permission from reference 133. Copyright 2009 American Chemical Society)

Pranath et al have reported enhanced hydrogen storage capacity at high pressure for MIL-101/SWCNT (single-walled
25 carbon nanotube) composites. $^{137}$ A layer-like structure is observed by TEM indicating growth of the MOF on the functionalised surface of SWCNTs and $\mathrm{N}_{2}$ adsorption isotherms reveal an increase in ultramicropores for the composite, as previously reported for MOF/GO and MOF/MWCNT materials. 30 For a composite of MIL-101 with 8 wt $\%$ SWCNTs, hydrogen uptake at 60 bar was increased by $44 \%$ at $77 \mathrm{~K}$ and $178 \%$ at 298 K over pure MIL-101.

$\mathrm{Li}^{+}$doping of MOFs has previously been shown to enhance gas-storage capacity ${ }^{138}$, particularly hydrogen, and this has been 35 explored further by Xiang et al who have reported the $\mathrm{Li}^{+}$doping of MOF/CNT composite materials. ${ }^{139}$ Highly admixed HKUST1/CNT composites were prepared from acid-treated MWCNTs and loaded with a Li-naphthalenide complex. Upon framework loading, the highly nucleophilic complex reacts with the $\mathrm{Cu}$ 40 bound water molecules of the paddlewheel SBUs to form a stable $\mathrm{Li}$ alkoxide species and naphthalene which is subsequently removed by washing. $\mathrm{At}^{+}{ }^{+}$loading levels in the range $0.07<$ $\mathrm{Li} / \mathrm{Cu}<0.12$, the structure of HKUST-1 was unchanged but at much higher levels PXRD indicated framework collapse. In 45 agreement with previous studies, gas storage capacity was increased in the presence of CNTs or $\mathrm{Li}^{+}$over the pure MOF, which is further enhanced for the Li-doped HKUST-1/CNT hybrids where $\mathrm{CO}_{2}$ and $\mathrm{CH}_{4}$ uptakes were increased, respectively, by $400 \%$ and $300 \%$ per effective surface area. Isosteric heat of 50 adsorption calculations, indicate that the very high enhancement observed for $\mathrm{CO}_{2}$ is attributed to an increased interaction with $\mathrm{Li}^{+}$. While this observation appears in accord with bulk MOFs containing low loadings of Li-alkoxide complexes ${ }^{140}$, the exact mechanism by which $\mathrm{Li}^{+}$can enhance interactions with hydrogen 55 in MOFs remains poorly characterised.

\section{MOFs at biopolymer interfaces}

There is now an ever expanding number of MOFs prepared from the building blocks of large naturally occurring macromolecules, including amino-acids ${ }^{16}$, oligopeptides ${ }^{10}$ and nucleobases. ${ }^{56,141}$

${ }_{60}$ Small endogenous bioactive molecules (e.g. fumarate, lactate and tartrate) have also been used to prepare chiral frameworks ${ }^{121,142,143}$ or increase their biocompatibility in combination with relatively benign metal ions for applications such as drug delivery. ${ }^{98,121}$ These so-called 'bioMOFs' have been 65 the subject of two excellent recent reviews. ${ }^{17,144}$

There still remain however a very limited number of MOF composite materials prepared by exploiting the rich functionality, processability and supramolecular self-assembly of naturally occurring biopolymers and protein cages. This chemistry is 70 significantly more developed for the coordination polymer Prussian blue $(\mathrm{PB})^{145}$ and its analogues, due to their facile preparation from water soluble components and interesting magnetic properties. ${ }^{146}$ These PB-biopolymer composites can however be taken as a model system to indicate the significant 75 opportunities available to MOFs in this area.

By analogy with the MOF/polymer composites described above, readily available polysaccharides such as chitosan have been used to prepare composite materials of cyanometallate network structures and to subsequently stabilise their colloidal 80 dispersions. ${ }^{147,148}$ Chitosan is a biocompatible polysaccharide bearing terminal amine functionality, which is easily solubilised 
in acidic media to form a cationic polyelectrolyte permitting its processing into membranes and beads. ${ }^{149,150}$

Guari et al have exploited the amine functionality of porous chitosan beads as a scaffold to prepare nanocomposites of 5 magnetic cyano-bridged coordination polymers $\mathrm{M}^{2+} /\left[\mathrm{Fe}\left(\mathrm{CN}_{6}\right)\right]^{3-}$
$(\mathrm{M}=\mathrm{Ni}, \mathrm{Cu}, \mathrm{Co}, \mathrm{Fe}){ }^{147}$ (Fig. 47I) TEM of the composites indicates a homogeneous dispersion of 2-3 nm cyanometallate particles within the chitosan matrix (Fig. 47IIa), and UV and IR spectra display characteristic bands corresponding to the bulk 10 cyanometallate networks.
(I)

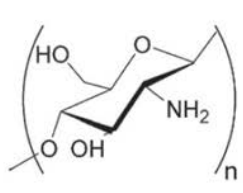

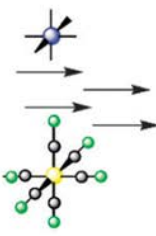

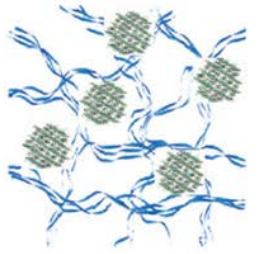

(III)

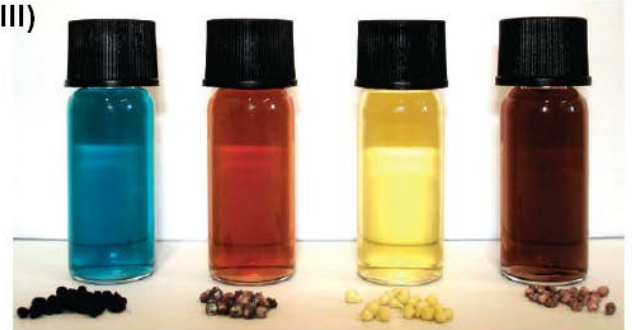

(II)
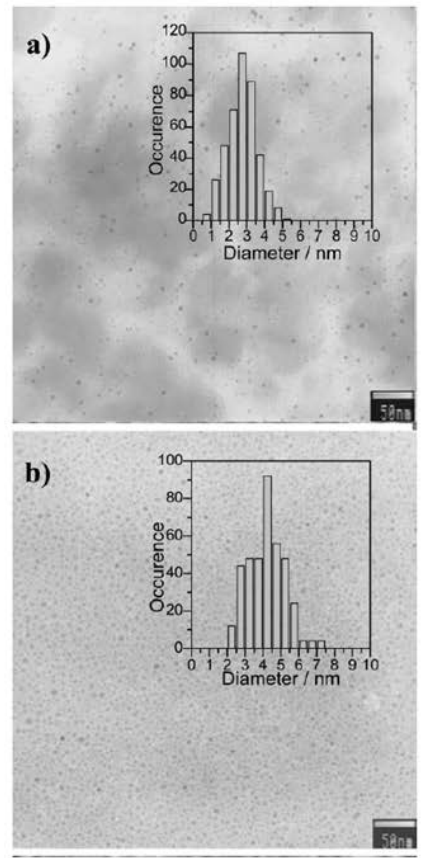

Fig. 47 (I) Schematic representation of the use of a porous chitosan matrix for the formation of cyano-bridged coordination polymers of general formula $\mathrm{M}^{2+} /\left[\mathrm{Fe}\left(\mathrm{CN}_{6}\right)\right]^{3-}(\mathrm{M}=\mathrm{Ni}, \mathrm{Cu}, \mathrm{Co}, \mathrm{Fe})$. (II) TEM images and size distributions of the cyanometallate nps in the chitosan matrix (a) and upon dispersion in acidic aqueous solution (b); note the increase in size due to the residual chitosan shell surrounding the nps upon dispersion. (III) Photograph of water

15 colloidal solutions of $\mathrm{M}^{2+} /\left[\mathrm{Fe}\left(\mathrm{CN}_{6}\right)\right]^{3-}$ and the corresponding chitosan beads for $\mathrm{M}=\mathrm{Fe}, \mathrm{Cu}$, Ni, Co going L-R) (Adapted from reference 146 and 147 with permission from, respectively, The Royal Society of Chemistry (RSC) for the Centre National de la Recherche Scientifique (CNRS) and the RSC)

)

Significantly, the nanocomposite beads can be solubilised in weak aqueous acidic media ( $\mathrm{pH} 4-5)$ to yield a stable colloidal 20 dispersion as shown in Fig. 47III, permitted by the advantageous properties of the biopolymer. The observed stability arises from a residual chitosan shell surrounding the cyanometallate nps to give a core-shell structure as evidenced by their increased size on dispersion (Fig. 47IIb), likely facilitated by strong interaction 25 with the biopolymer amine functionality. Similar results have been reported for luminescent sub-10 nm $\mathrm{Tb}_{2}(\mathrm{bdc})_{3}\left(\mathrm{H}_{2} \mathrm{O}\right)_{4} \mathrm{np}$ dispersions in the presence of PVP. ${ }^{151}$ The cyanometallate nanocomposite dispersions displayed spin glass-like magnetic behaviour arising from interparticle interaction.

$30 \mathrm{Gd}(\mathrm{III})$ cyanometallate/chitosan nanocomposites have also been prepared as potential contrast agents for MRI. ${ }^{148}$ Spherical non-aggregated particles of $3 \mathrm{~nm}$ diameter were dispersed in a chitosan matrix, which increased in size to $6 \mathrm{~nm}$ upon (acidic) dispersion. The composite np colloids displayed paramagnetic 35 properties and higher nuclear relaxivities than some clinically approved contrast agents. This approach has recently been extended to prepare ultra-small magneto-luminescent cyanometallate nps as bi-functional probes for combined MRI and optical imaging. ${ }^{152}$

40 Cyanometallate nps of general formula
$\operatorname{Ln}_{0.33}{ }^{3+} \mathrm{Gd}_{\mathrm{x}}{ }^{3+} /\left[\mathrm{Mo}(\mathrm{CN})_{8}\right]^{3-}(\mathrm{Ln}=\mathrm{Eu}(\mathrm{x}=0.34) ; \mathrm{Tb}(\mathrm{x}=0.35))$ homogeneously dispersed in porous chitosan matrices have been prepared in a stepwise fashion by first loading the biopolymer beads with a solution containing $\mathrm{Gd}^{3+}$ and $\mathrm{Ln}^{3+}$, followed by ${ }_{45}$ drying and immersion into a solution of the octacyanomolybdenum anion. ${ }^{152}$ As outlined for other cyanometallate/chitosan composites, stable colloidal dispersions of the cyanometallate nps are easily achieved at mildly acidic $\mathrm{pH}$. The resulting nanocomposite particles are $3-4 \mathrm{~nm}$ in size as 50 determined by (HR)TEM and are dispersible in both aqueous solution at neutral $\mathrm{pH}$ and physiological media.

The cyanometallate building blocks were carefully chosen to permit both magnetic $\left(\mathrm{Gd}^{3+}\right)$ and optical $\left(\mathrm{Ln}^{3+}\right)$ imaging, where the $\left[\mathrm{Mo}(\mathrm{CN})_{8}\right]^{3-}$ anion was specifically employed to prevent 55 quenching of the lanthanide luminescence. The aqueous colloidal nanocomposite dispersions exhibit bimodal emission at room temperature that can be controlled by the excitation wavelength. ${ }^{152}$ Under UV excitation red emission typical for $\mathrm{Ln}^{3+}$ intra- $4 f$ transitions dominate, but green emission from the 60 protective chitosan shell is observed under visible excitation suggesting a potential for multiplexed detection. In addition, the nuclear relaxivity measurements of $\mathrm{Ln}_{0.33}{ }^{3+} \mathrm{Gd}_{\mathrm{x}}{ }^{3+} /\left[\mathrm{Mo}(\mathrm{CN})_{8}\right]^{3-}$ reveal MRI performance comparable to commercial Gd-based 
positive contrast agents.

In-vivo studies of the multi-modal cyanometallate nanocomposites using confocal laser scanning microscopy (CLSM) revealed rapid internalisation of the particles by both 5 healthy (HUVEC and fibroblasts) and living cancer (HCT116 and Capan-1) cell lines. (Fig. 48) In all cases, the particles were efficiently localised into the lysosomes as a result of the endocytosis uptake pathway as confirmed by the merged images shown in Fig. 48 of np fluorescence and that of a lysosome 10 marker. Cell viability studies further indicated that all cells investigated were unaffected by the nanocomposites in the concentration range for effective imaging.

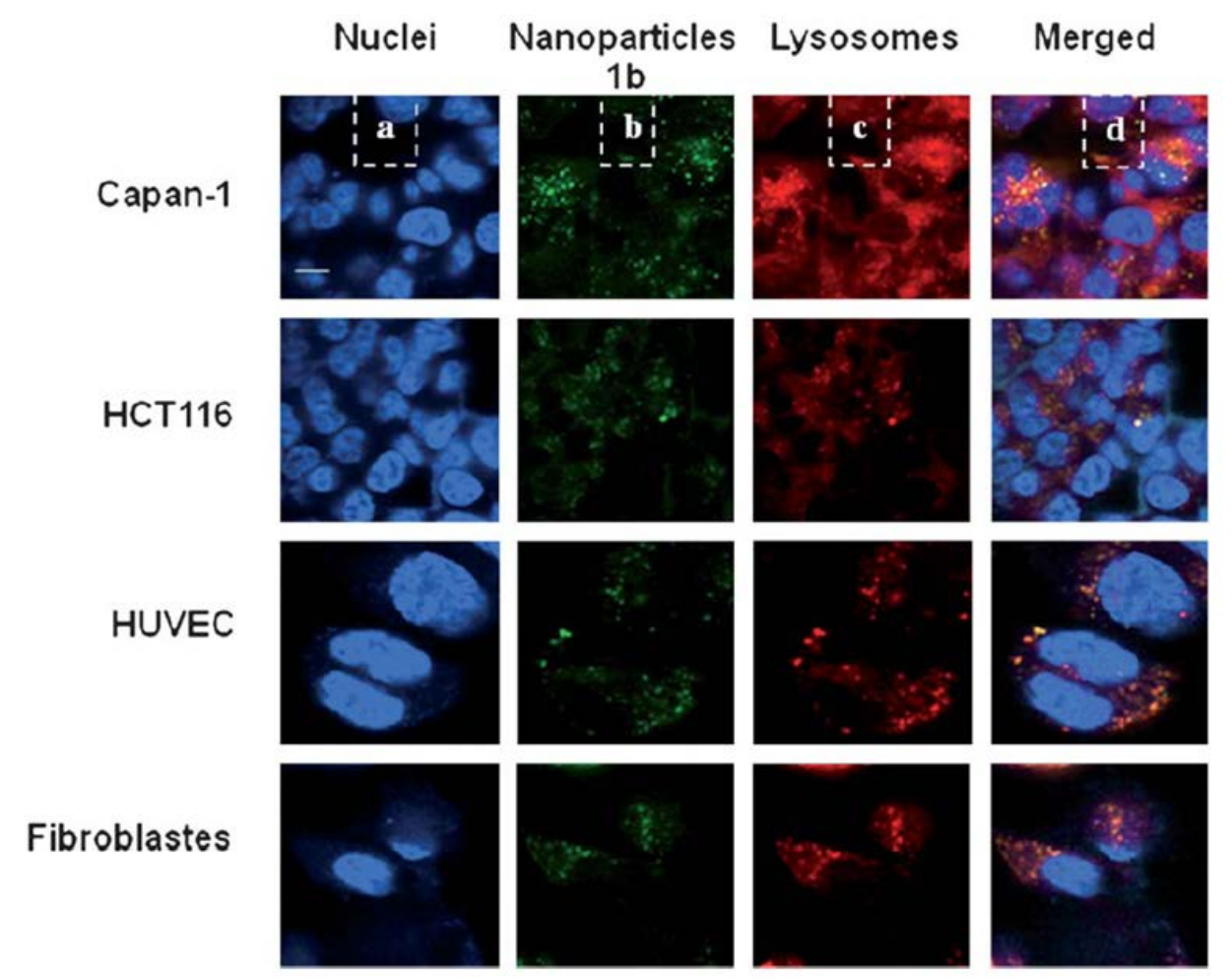

15 Fig. 48 Localization of nanoparticles of $\mathrm{Eu}_{0.33}{ }^{3+} \mathrm{Gd}_{\mathrm{x}}{ }^{3+} /\left[\mathrm{Mo}(\mathrm{CN})_{8}\right]^{3-}$ (labelled $1 \mathrm{~b}$ in the fig. legend) in living cancer (HCT-116 and Capan-1), or normal (HUVECs and fibroblasts) cell lines, incubated $6 \mathrm{~h}$ with the nanoparticles. Merged pictures represent the co-localization of the cyanometallate nanoparticles $\left(100 \mathrm{ng} \mathrm{mL}^{-1}\right)$ with the lysosomal marker (lysotracker, $50 \mathrm{nM}$ ). Images are representative of two independent experiments. Bars represent a scale of $4 \mu \mathrm{m}$. (Reprinted with permission from reference 152. Copyright 2011 Royal Society of Chemistry)

These studies suggest that nanocomposite formation and 20 processing of MOFs with soluble functional biopolymers is emerging as a promising strategy for the preparation of multifunctional nanosystems for combined therapeutic and diagnostic applications.

Cellulosic pulp fibres have also been used as an interface for 25 MOF growth to effectively prepare MOF-paper composites that could have application in routine low cost filtration applications. ${ }^{153}$ Küsgens et al prepared HKUST-1 in the presence of a range of pulp fibres, and found that MOF coverage was dependent on the lignin content remaining after (pulp) fibre 30 processing. As lignin content increased - as determined by a larger K-number for the pulp - greater MOF coverage was observed up to a recorded BET surface area of $314 \mathrm{~m}^{2} / \mathrm{g}$ for a HKUST-1/CTMP (where CTMP = chemithermomechanical pulp) composite $^{153}$. Unlike cellulose, lignin contains a large number of 35 carboxylate functionalities which clearly favour MOF growth as previously described for SAMs on Au. ${ }^{42}$

The supramolecular nature, reliable self-assembly and nanoscale dimensions of protein cages and virus capsids have long been exploited by materials chemists as confined reaction 40 vessels for the preparation of nanoscale objects ${ }^{154}$, and their facile functionalisation for higher level assembly. The uniformity of such cages allows excellent size control and monodispersity of the resulting materials and the functional groups presented at both the internal and external interfaces is attractive for the 45 introduction of multiple materials functions. Pre-eminent among these protein cages is ferritin ${ }^{155}$, a robust protein shell comprised of 24 subunits that self assemble into a spherical protein cage with an interior diameter of $8 \mathrm{~nm}$. In its natural state it contains an insoluble ferrihydrite mineral core that is easily removed to 50 leave the empty apo-ferritin (apo-F) shell. The apo-F core is readily reconstituted with a range of materials including metal and alloy nps ${ }^{156}$, although for coordination polymers only PB has been widely prepared in this way. ${ }^{145}$ By confining the growth of PB to the nanoscale it should become feasible to exploit single 55 magnetic domains of this material.

This approach has been further developed by Lucon et al to prepare a coordination polymer using $\mathrm{Cu}(\mathrm{I})$-catalysed click chemistry inside the related protein cage structure of small heat shock protein (sHSP). ${ }^{157}$ which is shown schematically in Fig. 49. 60 While not strictly a self-assembled or porous framework, this deserves mention here since an in depth understanding of the nature of the MOF/protein interface will be vital to future in-vivo 
biomedical applications of MOFs: such understanding can only come from the preparation of MOF/protein composite materials. Related to this strategically important goal is a recent study by Jung et al, who exploited the carboxylate-terminated surface of 5 MOF crystals for the bioconjugation of fluorescent proteins and a functional lipase which retained both its activity and enantioselectivity. ${ }^{158}$

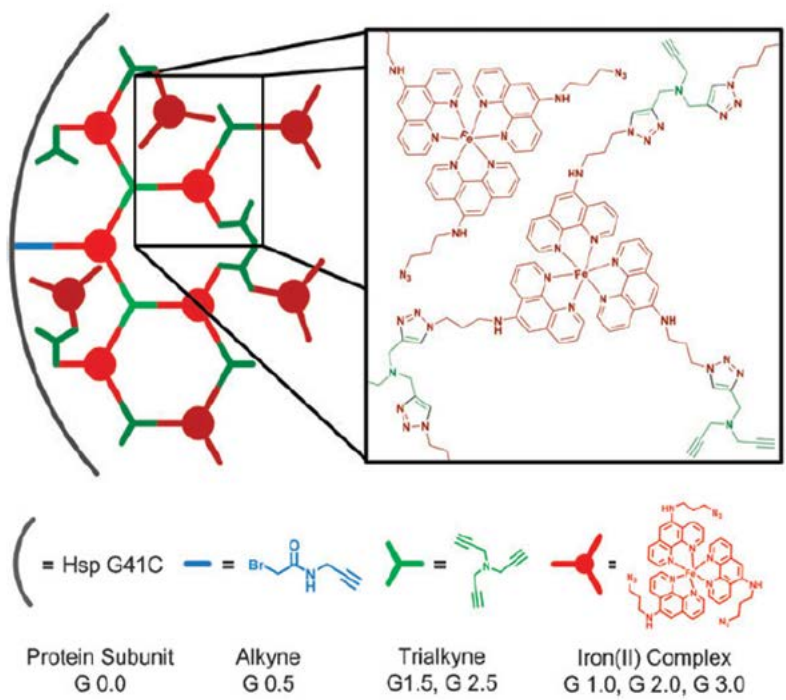

Fig. 49 Model scheme for the stepwise formation of a coordination 10 polymer inside the nanoscale cavity of small heat shock protein (sHSP, G41C) starting at the modified internal cysteine of each protein subunit. Each generation (G1.0, 2.0, 3.0) is added through the introduction of either an alkyne (G0.5) or azide $(\mathrm{G} 1.5,2.5)$ functional group containing compound permitting the click reactions to proceed. (Reprinted with 15 permission from reference 157. Copyright 2010 Royal Society of Chemistry)

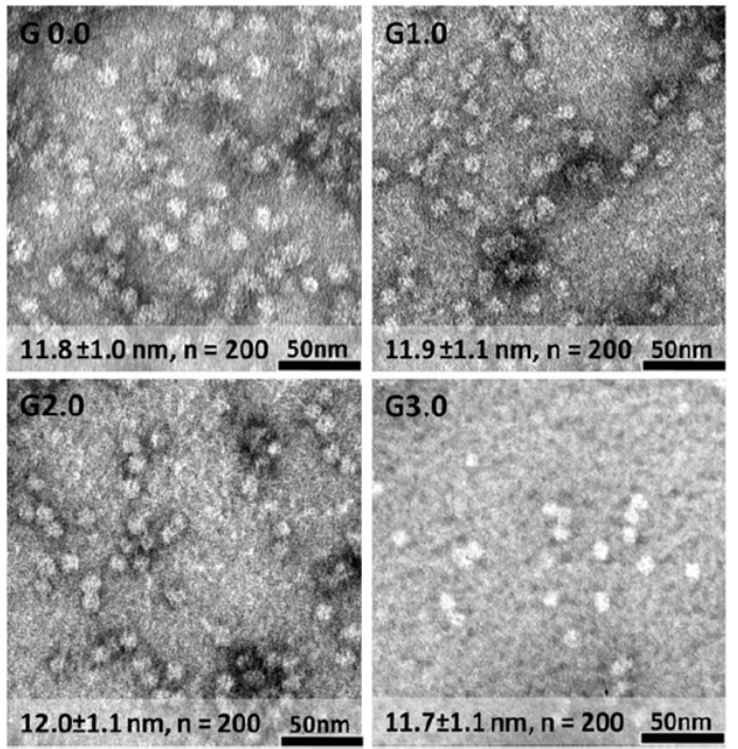

Fig. 50 Negative stain (2\% uranyl acetate) TEM images comparing the size of the native sHSP cage (G0.0) with successive generations of added $20 \mathrm{Fe}$ (II) coordination polymer (G1.0, G2.0, G3.0). Cage-like architectures of $\sim 12 \mathrm{~nm}$ diameter are seen in each case, confirming the protein cages remain intact during coordination polymer synthesis and that growth is confined to the protein interior. (Reprinted with permission from reference 157. Copyright 2010 Royal Society of Chemistry)

25 In the report by Lucon et al, internal cysteine residues of an engineered sHSP were used to introduce alkyne functionalities as growth points for a branched iron-phenanthroline (phen) coordination polymer. ${ }^{157}$ An octahedral Fe(II)(phen) $)_{3}$ complex with pendant azide groups was clicked into place in the presence 30 of $\mathrm{CuSO}_{4}$ /ascorbate followed by tripropargylamine (TPA) to permit further propagation. After 3 generations of the $\mathrm{Fe}(\mathrm{II})(\text { phen) })_{3}$ complex (two of TPA) were complete, no further growth was observed as the coordination polymer extended across the interior cavity of sHSP. The protein cage remains 35 intact throughout the entire polymerisation process as determined by TEM (Fig. 50), and the coordination polymer occupies approximately $20 \%$ of the available interior volume. In the absence of the protein cage, mixing of the reactants led to rapid precipitation of the bulk coordination polymer. While this 40 indicates that polymerisation occurred only within the protein interior, it perhaps more significantly demonstrates that insoluble extended coordination networks are easily solubilised in aqueous media by incorporation into robust potentially biocompatible protein vesicles. Such protein cages could offer protection for a 45 wide variety of MOFs to significantly extend their potential in applications where nanoscale aqueous dispersions are required.

\section{MOFs at MOF interfaces}

As clearly demonstrated at the beginning of this review, SAMs on a range of substrates can act as ordered functional interfaces 50 for highly oriented growth of polycrystalline MOF films ${ }^{20,24,32}$, and their single-crystal arrays. ${ }^{159}$ True epitaxy on the other hand is the growth of monocrystalline films on monocrystalline substrates, and is widely used in nanotechnology for the preparation of high quality semiconductor devices. ${ }^{160}$ The success 55 of this method relies on a close match or relationship between the lattice of the underlying substrate and that of the deposited material; however, heteroepitaxy - where the deposited film and substrate are of different composition - is possible if these conditions are met within certain tolerances. The single crystal 60 surfaces of MOFs have consequently been used as a functional interface for further MOF growth to prepare nested heteroepitaxial MOF@MOF structures, including systems with modular functionality. ${ }^{161}$

Given the requirement for a close lattice match between the 65 two components, isoreticular series ${ }^{4}$ of MOFs are ideal candidates for epitaxial growth where ligands and/or metals can be varied without disruption of the network topology yielding core-shell type crystals. ${ }^{162,163}$ (Fig. 51a) Preparation is extremely straightforward, and easily effected by adding fragments of the 70 core MOF crystal to a solvothermal synthesis of the shell MOF, and early work in this area was carried out on molecular crystals by Hosseini and co-workers. ${ }^{164,165}$ A recent review by Zacher et al explores further the surface chemistry at the MOF-liquid interface and potential growth mechanisms. ${ }^{30}$

75 Kitagawa and co-workers have explored heterometallic epitaxial growth of tetragonal $\left[\mathrm{M}_{2}\right.$ (ndc) ${ }_{2}$ (dabco) $]$ where $\mathrm{M}=$ $\mathrm{Zn}$ (II) for the substrate or core crystal, and $\mathrm{M}=\mathrm{Cu}(\mathrm{II})$ for the deposited shell crystal. ${ }^{162}$ (Fig. 51a) Optical microscopy of a 
cross-sectioned crystal clearly showed a colourless core and a green shell arising from the heterometallic nature of the MOF@MOF hybrid. Both core and shell were single crystals in their own right with the expected $P 4 / \mathrm{mmm}$ tetragonal space 5 group, and while there is a slight mismatch $(\leq 1 \%)$ in their unit cell parameters, this is sufficient to permit a detailed investigation of the structural relationship at the interface. The lattice match between the core and shell crystals is seemingly dependent on the crystal surface; for example, there is a near perfect match at the 10 (100) surface whereas rotational growth of two Miller domains of the shell lattice are observed at the (001) surface with an average rotational angle between them of $11.7^{\circ}$. (Fig. 51b) This likely occurs to maximise the coincidence between the two lattices, offsetting the small mismatch between them. It is noted however 15 that by employing a stepwise LbL growth method, perfect coincidence between these MOFs can be achieved when $\left[\mathrm{Zn}_{2}(\mathrm{ndc})_{2}\right.$ (dabco)] is grown on [001] oriented thin films of $\left[\mathrm{Cu}_{2}(\mathrm{ndc})_{2}\right.$ (dabco) $]$ supported on SAMs on Au. ${ }^{166}$
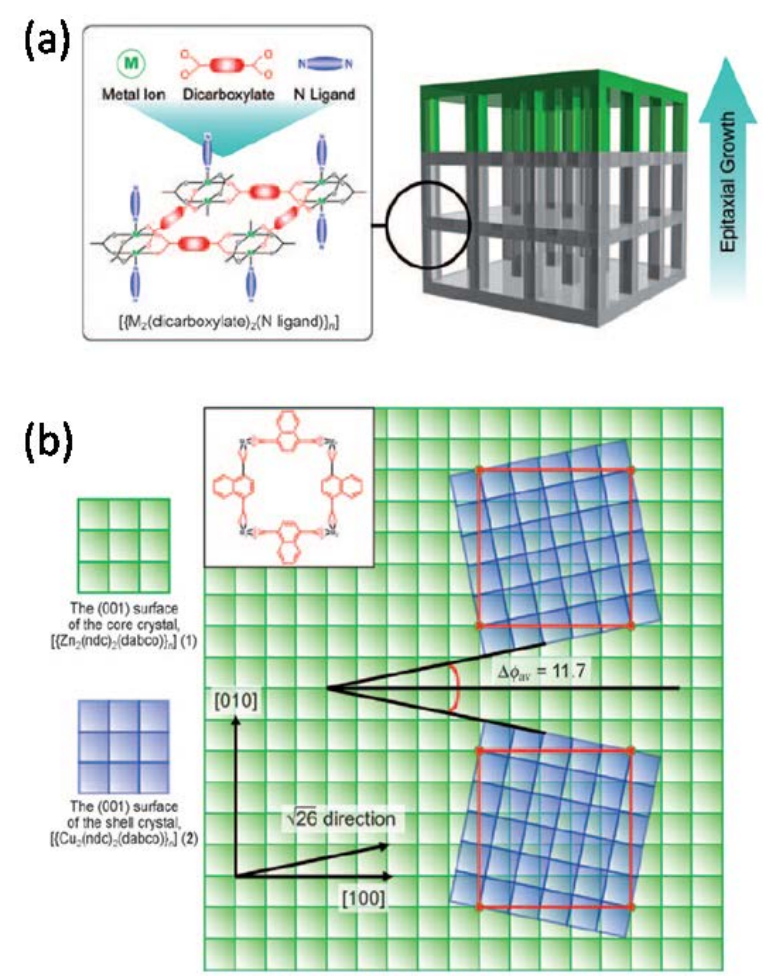

20 Fig. 51 (a) Schematic illustration of the heteroepitaxial growth of MOF@MOF structures with the $\left[\mathrm{M}_{2}(\mathrm{~L})_{2}(\mathrm{P})\right](\mathrm{L}=$ dicarboxylate; $\mathrm{P}=$ diamine pillar) isoreticular family. (b) Schematic model of the structural relationship between the core lattice $\left[\mathrm{Zn}_{2}(\mathrm{ndc})_{2}(\mathrm{dabco})\right]$ and the shell lattice $\left[\mathrm{Cu}_{2}(\mathrm{ndc})_{2}\right.$ (dabco)] on the (001) surface. The red lines indicate the 25 commensurate lattice between the core lattice and the shell lattice; the (5 $x$ 5) structure of the core crystal or the $(\sqrt{26} x \sqrt{2} 26)$ structure of the shell. Two Miller domains of the shell crystal are grown on the (001) surface of the core crystal while maintaining the rotational angle $\left(\Delta \varphi_{\mathrm{av}}=11.6518\right)$, which corresponds to the $\sqrt{2} 6$ direction of the (001) surface. The inset 30 shows the chemical structure of the (001) surface. (Adapted with permission from reference 162. Copyright 2009 Wiley-VCH)

The ligands rather than the metals have also been varied during heteroepitaxial growth of MOF hybrid materials. Koh et al exploited the widely studied $\left.\left[\mathrm{Zn}_{4} \mathrm{O} \text { (dicarboxylate) }\right)_{3}\right]$ IRMOF 35 series to prepare core-shell structures containing ligands of different functionality. ${ }^{163}$ The resultant hybrids exhibited good colour contrast arising from colourless IRMOF-1 ( $\mathrm{L}=1,4-$ benzenedicarboxylate) and orange IRMOF-3 (L = 2-amino benzene-1,4-dicarboxylate) and core-shell structures were 40 obtained both ways (viz. IRMOF-1 core/IRMOF-3 shell and vice versa). (Fig. 52) The hybrids retain their porosity and crystals with multiple layers were also prepared. Yoo et al further extended this approach by successfully preparing an

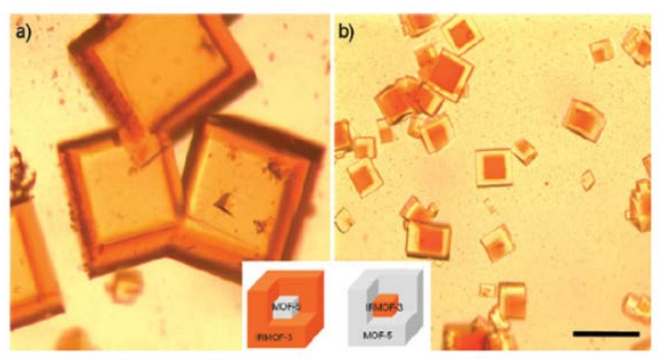

45 Fig.52 Microscope images of core-shell MOFs (a) IRMOF-3(shell)@IRMOF-1(core), (b) IRMOF-1(shell)@IRMOF-3(core). Scale bar, 200 $\mathrm{mm}$. (Reprinted with permission from reference 163. Copyright 2009 Royal Society of Chemistry)

IRMOF-3 film through LbL growth from an IRMOF-1 seed layer 50 deposited on a porous alumina support ${ }^{167}$, opening up the opportunity to prepare multifunctional supported MOF membranes for combined catalysis and separation.

The modular $\left[\mathrm{M}_{2}(\mathrm{~L})_{2} \mathrm{P}\right]$ structure (Fig. 51a) is extremely versatile due to the simultaneous presence of both carboxylate $(\mathrm{L})$ 55 and amine (P) linkers and is thus an ideal system to explore the possibilities afforded by the heteroepitaxial approach Kitagawa and co-workers have recently devised a strategy to prepare functional MOF@MOF hybrid systems through sequential ligand functionalisation ${ }^{161}$, which can also be realised in a face-selective 60 manner. ${ }^{168}$ This permits the controlled location and separation of individual ligand functionalities on the $\mathrm{L}$ component which would otherwise be scrambled in a homogenous phase prepared by simple mixing of the two ligands at the synthesis stage. Heteroepitaxy was thus employed to form core-shell and end65 capped MOF@MOF structures with spatially separated porous networks of differential pore size.

Core-shell structures were prepared from $\left[\mathrm{Zn}_{2}(\mathrm{~L})_{2}(\mathrm{dabco})\right]$ where $\mathrm{L}=$ bdc was used for the core crystal and $\mathrm{L}=$ bulky adc (9,10-anthracene dicarboxylate) for the shell, resulting in a clear 70 difference in the pore dimensions between the two components (core 7.5 x $7.5 \AA$ and 5.3 x $3.2 \AA$; shell 4.5 x $2.7 \AA$ and 1.7 x 1.7 $\AA$ ), allowing the hybrid to act as an efficient and highly selective extractor system. ${ }^{161}$ (Fig. 53) The hybrid crystal was found to selectively adsorb and concentrate cetane (n-hexadecane) from 75 the branched isomer isocetane (2,2,4,4,6,8,8-heptamethylnonane) as determined by GC-MS, even when the concentration of cetane was $<1 \%$. This arises from the molecular sieving effect of the small pores of the defect free shell, which do not allow the isocetane to enter the core. The large pores of the core crystal 80 alone are unselective and concentrate both linear and branched isomers; however, only linear cetane is concentrated in the core crystal of the hybrid further confirming the selectivity of the shell.

By using diamine pillaring ligands $(\mathrm{P})$ of different length 
rather than the functionalised carboxylates outlined above, it is possible for $\left[\mathrm{Zn}_{2}(\mathrm{ndc})_{2} \mathrm{P}\right]$ phases to undergo anisotropic heteroepitaxial growth to form end-capped rectangular crystals. ${ }^{168}$ This arises due to the tetragonal nature of the structure, where 5 four [100] surfaces contain one carboxylate layer ligand and one pillaring diamine whereas the two [001] surfaces contain only layer ligands. Reaction of

a)
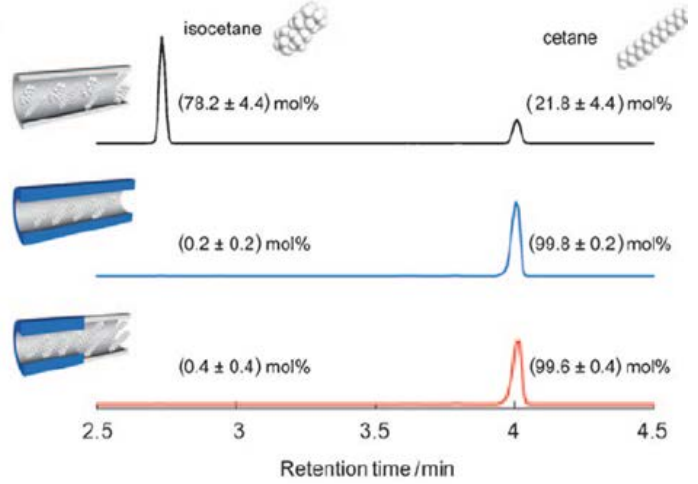

b)

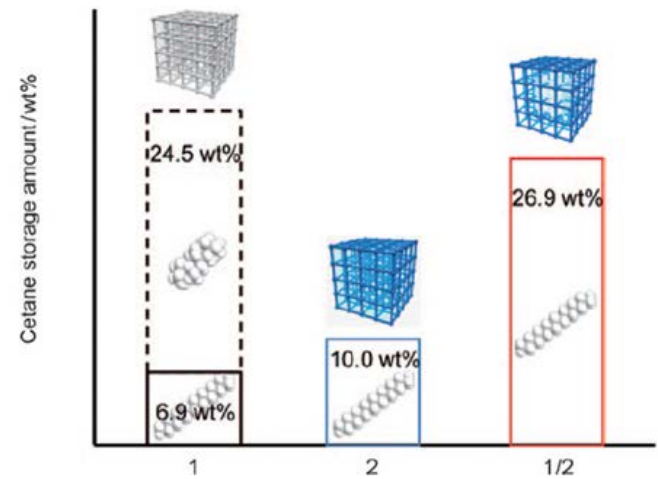

Fig. 53 The selective adsorption of cetane over isocetane by core/shell $10\left[\mathrm{Zn}_{2}(\mathrm{~L})_{2}(\right.$ dabco) $]$ structures (where $\mathrm{L}=$ bdc for the core crystal and $\mathrm{L}=$ bulky adc $(9,10$-anthracene dicarboxylate for the shell) with differential pore sizes from mixtures with a cetane/isocetane ratio of $1: 100$ as determined by GCMS (a). The core-shell crystal only accumulated cetane (bottom) owing to the small pores of the shell crystal (middle); in 15 contrast, isocetane was preferentially adsorbed by crystals of core only (top). (b) The amount of cetane stored in core only, shell only, and core/shell structures when using a 1:100 mixture of cetane/isocetane. (Reprinted with permission from reference 161. Copyright 2011 Wiley$\mathrm{VCH})$

${ }_{20}\left[\mathrm{Zn}_{2} \text { (ndc) }\right)_{2}$ (dabco) $]$ in the presence of the second much longer pillaring diamine, dpndi ( $N, N^{\prime}$-di(4-pyridyl)-1,4,5,8naphthalenetetracarboxydiimide), results only in growth at the [001] surfaces. As the same layer ligand is used in both crystals there is a clear lattice match within the layers; however, no 25 interlayer lattice match can be achieved at [100] due to the significant difference in size between the two pillars (the $c$ unit cell parameter for the two crystals are 9.61 for dabco and $22.46 \AA$ for dpndi) prohibiting growth in this direction. Diffraction studies of the end-capped crystals reveal true epitaxial coincidence of the 30 lattices indicating alignment of the pore structures.

There are clear advantages to preparing heterogeneous
MOF@MOF hybrids using modular platforms, permitting effective combination of disparate or contraindicated functionalities. In principle, several different functionalities can 35 be coupled together in specific sequences optimising system performance for complex transformations, including MOF-based cascade reaction and separation systems.

MOF surfaces have also been used to support organic monolayers through ligand exchange processes ${ }^{169}$, permitted by

40 the relative lability of coordination bonds. The only requirement here is that the molecules used for the monolayer should bear the same functionality as the framework linkers as typically exploited for growth on SAMs, allowing them to exchange directly with the surface ligands. Under carefully controlled reaction conditions, 45 Kondo et al have prepared monolayers of benzoate functionalised bodipy (boron dipyrromethane) dyes on the carboxylate terminated surfaces of $\left[\mathrm{Zn}_{2}(\mathrm{~L})_{2}(\right.$ dabco $\left.)\right]$ ( $\mathrm{L}=$ bdc or ndc) and HKUST-1. ${ }^{169}$ Tetragonal $\left[\mathrm{Zn}_{2}(\mathrm{~L})_{2}\right.$ (dabco)] only has four [100] carboxylate terminated surfaces which can be modified with a 50 dye monolayer (Fig. 54, left), and this is confirmed by confocal laser scanning microscopy (CLSM) and face index analysis; the remaining amine terminated [001] surfaces remain unmodified. (Fig. 54, middle/right) This is the reverse of the anisotropic heteroexpitaxy described above that results in end-capping of the 55 [001] surfaces only. A similar observation was made for monolayer formation on cubic HKUST-1: all faces of the octahedral crystals are carboxylate terminated [111] surfaces and hence support a dye monolayer. In all cases, the structure of the monolayer will be determined by the lattice of the underlying 60 MOF substrate.

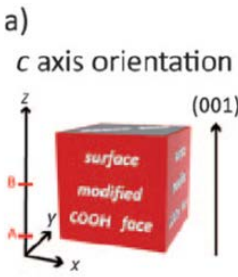

b)

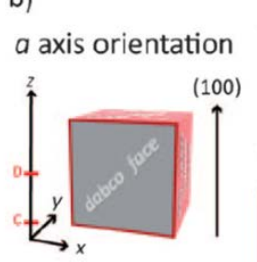

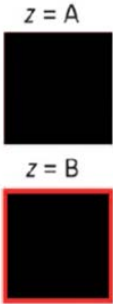
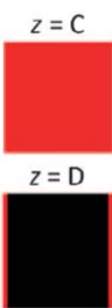
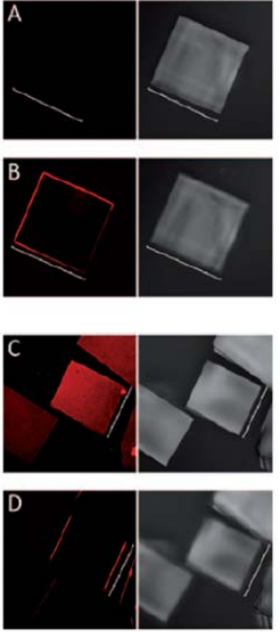

Fig. 54 Representations of bodipy surface-modified crystals of tetragonal $\left[\mathrm{Zn}_{2}(\mathrm{~L})_{2}\right.$ (dabco)] (left), CLSM images (middle) and transmission images (right) of the crystals at $\mathrm{z}=\mathrm{A}$ and $\mathrm{z}=\mathrm{B}$ in a) the $\mathrm{c}$ axis orientation and $\mathrm{b}$ ) 65 the a axis orientation. Scale bars: A, $180 \mu \mathrm{m}$; B, $190 \mu \mathrm{m}$; C, $130 \mu \mathrm{m}$; D, $130 \mu \mathrm{m}$. (Reprinted with permission from reference 169. Copyright 2010 Wiley-VCH)

\section{MOFs at liquid crystal interfaces}

Amphiphilic species such as surfactants and block copolymers 70 readily assemble into mesoscopic micellar and liquid crystalline phases above their critical micelle concentration. ${ }^{170}$ These soft 
supramolecular assemblies have been widely used as templates for the preparation of mesoporous materials of broad technological relevance. ${ }^{171}$ Condensable inorganic precursors are typically combined with the amphiphile resulting in their co5 assembly. Although this co-assembly can occur under a range of experimental conditions (e.g. temperature, $\mathrm{pH}$ ), its success ultimately relies on the interfacial interactions between the polar fragments of the amphiphile and the often charged inorganic species. After initial co-assembly, an aging step leads to 10 condensation of the inorganic precursors to form an inorganic/organic hybrid which is calcined to remove the organic template leaving a continuous porous inorganic replica of the mesophase.

Many examples of silicas and zeolites with ordered cubic and 15 hexagonal mesoporosity ${ }^{172}$ and hybrid periodic mesoporous organosilicas (PMOs) ${ }^{173}$ have already been successfully prepared in this way, but their functionality could be further enhanced if similar mesoporous phases could be prepared from extended coordination networks, by incorporating both transition metal 20 properties and organic functionality and flexibility. ${ }^{174}$ Further, and similar to the MOF@polymer systems outlined previously $^{31,103}$, the resulting hierarchical porosity will combine unhindered mass transport through the mesopores and tunable micropore functionality and selectivity. The ordered mesopores 25 may also provide new opportunities for the interaction of MOFs with larger biomolecular species. Such mesoporous MOFs are thus a desirable synthetic target for a range of novel applications.
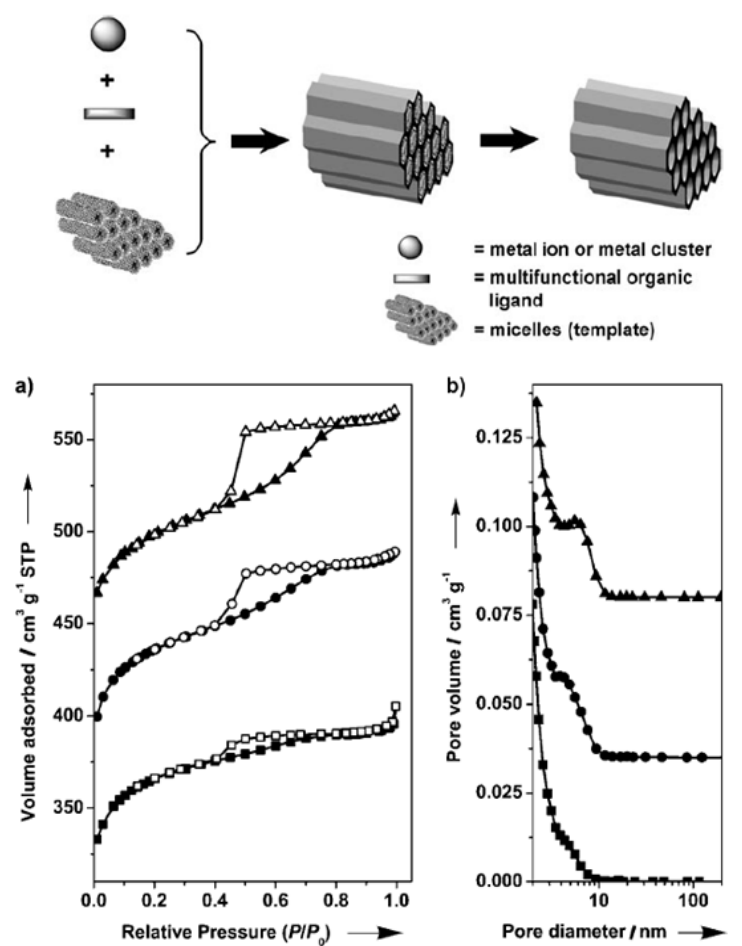

Fig. 55 Upper: Schematic representation of the mesoscale liquid crystal 30 templating of MOFs; the resulting materials have microporous walls due to the inherent porosity of the MOF itself. Lower (a) $\mathrm{N}_{2}$ adsorption isotherms (closed adsorption; open, desorption) and (b) corresponding pore size distributions obtained by the Barrett-Joyner-Halenda (BJH) method for templated HKUST-1 using $0.15(\square), 0.3(\circ)$ and $0.6(\Delta)$ molar 35 equivalents of CTAB (cetyl trimethyl ammonium bromide) per $\mathrm{Cu}^{2+}$. All curves in (b) are offset for clarity. (Reprinted with permission from reference 175. Copyright 2008 Wiley-VCH)

Cationic quaternary amine-based surfactant assemblies are commonly used for the preparation of mesoporous silicas, and ${ }_{40}$ Qiu et al have followed a similar strategy to access MOFs with combined micro- and mesoporosity. ${ }^{175}$ Cetyl trimethyl ammonium bromide (CTAB) was added to standard HKUST-1 solvothermal synthesis mixtures at various molecular ratios to template mesoporosity within the microporous framework as the 45 metal ions and ligands assemble around the surfactant micelles. (Fig. 55, upper) The CTAB template was subsequently removed from the as-made HKUST-1/CTAB composite by washing in ethanol, to yield the desired mesoporous MOFs. Commensurate with the two porous regimes nitrogen adsorption isotherms so intermediate between type I (microporosity) and IV (mesoporosity) were recorded, and the mesopore contribution increased with CTAB content up to a mesopore diameter of 5.6 $\mathrm{nm}$ for $\mathrm{Cu} / \mathrm{CTAB}=1.6$. (Fig. 55, lower) In fact, mesoporosity could be increased up to a pore size of $31 \mathrm{~nm}$ by adding 55 hydrophobic 1,3,5-trimethylbenzene (TMB) to the synthesis: TMB acts as a swelling agent to increase micelle size thus increasing mesopore diameter.

Small angle x-ray diffraction data of mesoporous HKUST-1 shows an absence of long range order, which is further confirmed 60 by TEM images that indicate formation of a disordered wormlike mesoporous network. ${ }^{175}$ This lack of long range order likely arises from the effect of the reaction temperature on the micellar assembly combined with the nature of the electrostatic interactions with deprotonated carboxylate ligands and other 65 anionic species at the interface during co-assembly. A range of interactions are possible between amphiphiles and precursor species during the assembly of mesoporous materials, including electrostatics, hydrogen bonding and anion and metal-mediated contacts, and these all need to be explored in suitable solvents in 70 order to optimise the template derived mesoporous ordering of MOF systems.

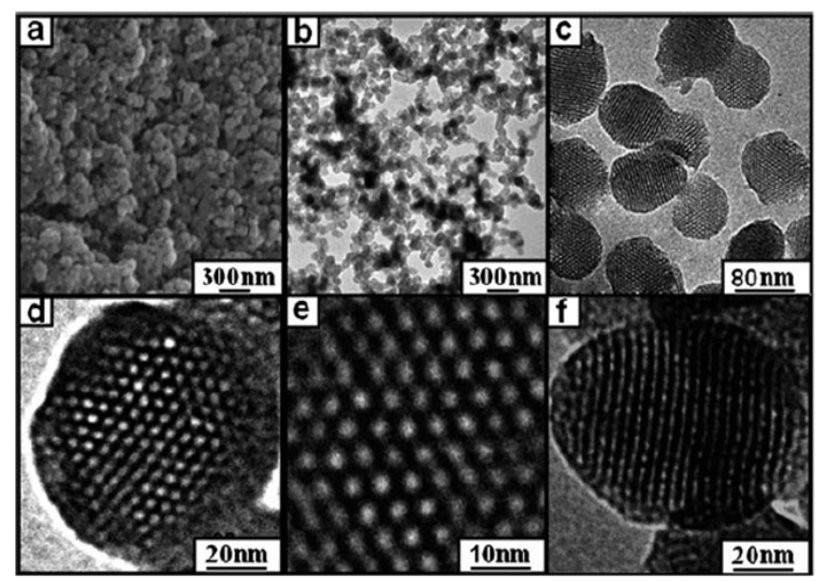

Fig. 56 (a) SEM and (b-f) TEM images of mesoporous MOF nanospheres formed using a ternary ionic liquid/ $\mathrm{scCO}_{2} /$ surfactant reaction system. The 75 spheres are $80 \mathrm{~nm}$ in diameter, and hexagonally ordered mesopores are clearly present. The pore size is $3 \mathrm{~nm}$ and the wall thickness is $2.5 \mathrm{~nm}$. (Reprinted with permission from reference 177. Copyright 2011 Wiley$\mathrm{VCH})$ 
Ordered mesoporous MOF nanospheres reminiscent of the mesoporous silica nps advanced as potential drug delivery systems ${ }^{176}$ have recently been prepared using a ternary ionic liquid/scCO $\mathrm{SC}_{2}$ surfactant reaction system. ${ }^{177}$ (Fig. 56) A 5 supramolecular surfactant assembly of $N$-ethyl perfluorooctylsulfonamide is formed in an emulsion type system where the polar head groups interact with an ionic liquid continuous phase containing $\mathrm{Zn}(\mathrm{II})$ and bdc, and the fluorous tales with a $\mathrm{scCO}_{2}$ internal phase. After reaction at $80{ }^{\circ} \mathrm{C}$ for 48 10 hrs and template removal, ordered mesoporous nanospheres of a microporous MOF of nominal composition [Zn(bdc)] are obtained. The spheres were approximately $80 \mathrm{~nm}$ (TEM, DLS) in diameter (Fig. 56) with a $\mathrm{N}_{2}$ derived mesopore size distribution centred at $3.6 \mathrm{~nm}$. This is an important advance for the 15 preparation of controllably nanoscale mesoporous MOFs, but the synthesis procedure involving ionic liquids and $\mathrm{scCO}_{2}$ may ultimately limit its scale-up and hence practical use.

Given the differences in self-assembly between silica and MOFs which could lead to difficulties in templated mesoporous 20 MOF synthesis, an alternative strategy is to prepare mesoporous MOF-oxide composite materials. Using a block copolymer template, Górka et al have prepared mesoporous HKUST-1/SiO and alumina composite materials in a one pot microwave assisted hydrothermal synthesis. ${ }^{27}$ Powder X-ray diffraction indicates that 25 although formation of the templated mesoporous oxides does not generally prohibit HKUST-1 composite formation, the synthesis is sensitive to $\mathrm{Al} / \mathrm{Cu}$ or $\mathrm{Si} / \mathrm{Cu}$ ratio as evidenced by the presence of competing phases. Nitrogen adsorption measurements of the HKUST-1/oxide composites reveal significant microporosity 30 arising from the MOF component and type IV behaviour in the high pressure region indicative of mesoporosity; however, it is not clear if this mesoporosity is ordered as no diffraction data below $2 \theta=5^{\circ}$ were reported. Some differences in adsorption are revealed between the HKUST-1/silica and HKUST-1/alumina 35 composites as expected given previous reports regarding the compatibility of the two components. ${ }^{58}$ Adsorption behaviour of the materials is however different from a physical mixture of the MOF and mesoporous oxide, revealing true composite formation. This is further confirmed by SEM and elemental mapping studies 40 for HKUST-1/alumina $(\mathrm{Al} / \mathrm{Cu}=2)$.

\section{Conclusions and future perspective}

One of the most common methods to prepare MOF-based thin films and composite materials is to grow or deposit these onto appropriately functionalised solid interfaces (supports). ${ }_{45}$ Consequently, the nature of the interface is absolutely vital not only to their preparation, but also to influence composite properties. SAMs of diverse functionality on Au substrates have revealed the importance of the interface on the nucleation, growth kinetics, and orientation of thin MOF films and these 50 fundamentals have been applied to a diverse range of supports including oxides, carbon, polymers, other MOFs etc as outlined in this review. The nature of the interface between the MOF and the support also plays a role in composite performance; for example, good adherence between the MOF and a polymeric 55 support in MMMs is vital if non-selective voids/defects are to be eliminated, and ultra-microporosity may also be introduced between the two components to enhance physisorption processes.
It is absolutely clear from this that an understanding of the interactions present at the interface are required in order to 60 determine meaningful structure-property relationships and design the next generation of high performance MOF composite materials and devices. It is not always easy to study these interfacial interactions but the ability to prepare MOF thin films not only allows us to form new composite materials, but provides 65 an opportunity to use the full gamut of surface science techniques to study the interface itself. This will be facilitated by molecular simulation and thus it is expected that fruitful collaborations with computational chemists will become increasingly common in this rapidly developing research area.

70 Composite materials prepared by the growth or deposition of MOFs at interfaces are steadily increasing in number and diversity of configuration. It is clear from this review however, that the vast majority of MOF-based composites and thin films prepared this far are dominated by a small number of well studied 75 systems e.g. MOF-5 (and the extended IRMOF series), HKUST1, MIL-n series, ZIF family and $\left[\mathrm{M}_{2}(\mathrm{~L})_{2}(\mathrm{P})\right]$; this is further borne out in the recent review by Bétard and Fischer ${ }^{32}$ which provides a useful inventory of those MOF families prepared as thin films and other coatings. This of course is a pragmatic approach to so demonstrate proof-of-principle given the range of synthetic methods reported for their controlled preparation, and their well understood porous behaviour allowing easy comparisons to the bulk MOFs. As a direct result of this groundwork, the stage is now set for the preparation of diverse MOF-based composite 85 materials with significantly enhanced properties using tailormade MOFs of demonstrable potential for a given application. For example, controllably nanoscale MOFs that have shown potential in drug delivery and diagnostics are highly likely to be combined with biopolymer interfaces to increase their 90 biocompatibility and aqueous solubility, further advances in separation science will be made by preparing MOF composite chromatography stationary phases from enantiomerically pure large pore frameworks and highly sensitive and multi-responsive sensing devices will be realised by combining MOFs of 95 demonstrably high selectivity and luminescent or magnetic behaviour with interfaces that can provide a suitable readout.

The desirable materials targets outlined above clearly build on the work previously carried out in MOF thin films and composites, but what about further perspectives for this field? 100 Recent work has exploited solution rather than solid interfaces to prepare MOFs; for example, formation of MOF nanofibres under conditions of laminar flow in microfluidic devices ${ }^{178}$ and the formation of thin MOF shells (capsules) displaying selective permeation around liquid droplets that act as a template. ${ }^{179}$ Using 105 the latter technique, hollow MOF-polymer composites with enhanced mechanical strength were also prepared. Free-standing oriented MOF films have also been prepared using a solid interface as a nucleation and growth template only, followed by removal of the well-defined polycrystalline MOF islands. ${ }^{180}$

110 Natural biomineralisation processes rely on interfacial growth and recognition at typically organic interfaces ${ }^{181}$, and while some of this has already been realised with MOF growth at SAMs and heteroepitaxial growth at (other) MOF interfaces it is undeniable that further significant opportunities exist in this area. When 115 mineralisation is under strict biological control, this highly 
regulated process can produce materials with unprecedented levels of structural complexity and finely tuned mechanical properties. The mechanisms that allow for such high levels of control regulate the chemical, spatial, structural, morphological 5 and constructional aspects of the system, which often involves compartmentalisation of the reaction space by some supramolecular assembly in a process termed 'boundaryorganised mineralisation'. Morphological control is a more complex situation, afforded by an organic supramolecular 10 boundary that changes shape with time, such that the developing inorganic phase perturbs the matrix to orient growth along specific directions. Such high degrees of control over synthesis and hierarchical levels of structure and functionality over multiple length scales will allow these highly ordered nanoporous 15 materials to be more easily processed, increasing their relevance and ability to address key societal and technological challenges.

The wider concepts of biomineralisation ${ }^{181}$ and the control of chemistry within well-defined synthetic and natural compartments are extremely attractive to control the size, shape 20 and orientation of MOFs. Surprisingly, very little biomimetic synthesis of MOFs in compartments other than reverse microemulsions has been reported ${ }^{22}$, despite the opportunity for high-gain in terms of advanced applications and processability. The programmable construction of MOF np building blocks using 25 biological recognition ${ }^{182}$ will also permit higher levels of controlled assembly beyond the first coordination sphere of the SBUs. Many of these concepts have not yet been applied directly to MOFs, and will clearly require the development of new chemistries. $^{183}$ Consequently, MOF-based composite materials 30 and the hitherto unexplored methods by which to prepare them remain an area rich in opportunity to be exploited by MOF chemists, surface scientists and those working in related fields such as nanoscience and biomineralisation.

\section{Acknowledgements}

${ }_{35}$ DB gratefully acknowledges the European Research Council for funding under grant ERC-StG-2010-BIOMOF-258613.

\section{References}

${ }^{a}$ Department of Chemistry, University of Liverpool, Crown Street, Liverpool L69 7ZD, UK. Fax: +44(0)151 794 3588; Tel: +44(0)151 794 40 3479; E-mail: deg5y@liverpool.ac.uk

1 S. Kitagawa, R. Kitaura and S. Noro, Angew. Chem. Int. Ed., 2004, 43, 2334.

2 G. Férey, Chem. Soc. Rev., 2008, 37, 191.

453 C. Janiak and J. K. Vieth, New J. Chem., 2010, 34, 2366.

4 M. Eddaoudi, J. Kim, N. Rosi, D. Vodak, J. Wachter, M. O’Keeffe and O. M. Yaghi, Science, 2002, 295, 469.

5 MOF special issue, Chem. Soc. Rev. 2009, 38, 1201 (19 articles).

6 Q. Li, W. Zhang, O. Š. Miljanić, C.-H. Sue, Y. L. Zhao, L. Liu, C. B.

$50 \quad$ Knobler, J. F. Stoddart and O. M. Yaghi, Science, 2009, 325, 855.

7 Z. Wang and S. M. Cohen, Chem. Soc. Rev., 2009, 38, 1315.

8 S. Horike, S. Shimomura and S. Kitagawa, Nat. Chem., 2009, 1, 695

9 X. Zhao, B. Xiao, A. J. Fletcher, K. M. Thomas, D. Bradshaw and M. J. Rosseinsky, Science, 2004, 306, 1012.

5510 J. Rabone, Y. F. Yue, S. Chong, K. C. Stylianou, J. Bacsa, D. Bradshaw, G. R. Darling, N. G. Berry, Y. Z. Khimyak, A. Y. Ganin, P. Wiper, J. B. Claridge and M. J. Rosseinsky, Science, 2010, 329, 1053.
11 C. Serre, C. Mellot-Draznieks, S. Surblé, N. Audebrand, Y. Filinchuk and G. Férey, Science, 2007, 315, 1828.

12 X. Lin, I. Telepeni, A. J. Blake, A. Dailly, C. M. Brown, J. M. Simmons, M. Zoppi, G. S. Walker, K. M. Thomas, T. J. Mays, P. Hubberstey, N. R. Champness and M. Schröder, J. Am. Chem. Soc., 2009, 131, 2159.

6513 R. Bannerjee, A. Phan, B. Wang, C. Knobler, H. Furukawa, M. O’Keeffe and O. M. Yaghi, Science, 2008, 319, 939.

14 A. Corma, H. Garcia and F. X. Llabre i Xamena, Chem. Rev., 2010, 110, 4606.

15 J.-R. Li, R. J. Kuppler and H.-C. Zhou, Chem. Soc. Rev, 2009, 38, 1477.

16 R. Vaidhyanathan, D. Bradshaw, J.-N. Rebilly, J. P. Barrio, J. A. Gould, N. G. Berry and M. J. Rosseinsky, Angew. Chem. Int. Ed., 2006, 45, 6495.

17 A. C. McKinlay, R. E. Morris, P. Horcajada, G. Férey, R. Gref, P.

75 Couvreur and C. Serre, Angew. Chem. Int. Ed., 2010, 49, 6260.

18 J. D. Rocca and W. Lin, Eur. J. Inorg. Chem., 2010, 3725.

19 B. Chen, L. Wang, Y. Xiao, F. R. Fronczek, M. Xue, Y. Cui and G. Qian, Angew. Chem. Int. Ed., 2009, 48, 500.

20 D. Zacher, O. Shekhah, C. Wöll and R. A. Fischer, Chem. Soc. Rev. $80 \quad 2009,38,1418$.

21 A. Carné, C. Carbonell, I. Imaz and D. Maspoch, Chem. Soc. Rev., 2011, 40, 291.

22 W. Lin, W. J. Rieter and K. M. L. Taylor, Angew. Chem. Int. Ed., 2009, 48, 650.

8523 I. Imaz, J. Hernando, D. Ruiz-Molina and D. Maspoch, Angew. Chem. Int. Ed., 2009, 48, 2325.

24 O. Shekhah, J. Liu, R. A. Fischer and C. Wöll, Chem. Soc. Rev. 2011, 40, 1081.

25 C. Carbonell, I. Imaz and D. Maspoch, J. Am. Chem. Soc., 2011, 133, $90 \quad 2144$

26 F. Schröder and R. A. Fischer, Top. Curr. Chem., 2010, 293, 77.

27 J. Górka, P. F. Fulvio, S. Pikus and M. Jaroniec, Chem. Commun. 2010, 6798.

28 W. J. Rieter, K. M. Pott, K. L. M. Taylor and W. Lin, J. Am. Chem. 95 Soc, 2008, 130, 11584.

29 F. Wang, Z.-S. Liu, H. Yang, Y.-X. Tan and J. Zhang, Angew Chem. Int. Ed., 2011, 50, 450.

30 D. Zacher, R. Schmid, C. Wöll and R. A. Fischer, Angew. Chem. Int Ed., 2011, 50, 176.

10031 L. D. O’Neill, H. Zhang and D. Bradshaw, J. Mater. Chem., 2010, 20, 5720.

32 A. Bétard and R. A. Fischer, Chem. Rev., available on-line Sept 2011 under dx.doi.org/10.1021/cr200167v.

33 S. Hermes, F. Schröder, R. Chelmowski, C. Wöll and R. A. Fischer, 105 J. Am. Chem. Soc., 2005, 127, 13744.

34 Y. Yoo and H.-K. Jeong, Chem. Commun., 2008, 2441.

35 R. Ameloot, L. Stappers, J Fransaer, L. Alaerts, B. F. Sels and D. E. De Vos, Chem. Mater., 2009, 21, 2580.

36 P. Horcajada, C. Serre, D. Grosso, C. Boissiere, S. Perruchas, C. $110 \quad$ Sanchez and G. Férey, Adv. Mater., 2009, 21, 1931.

37 E. Biemmi, C. Scherb and T. Bein J. Am. Chem. Soc., 2007, 129, 8054.

38 O. Shekhah, H. Wang, S. Kowarik, F. Schreiber, M. Paulus, M. Tolan, C. Sternemann, F. Evers, D. Zacher, R. A. Fischer and C. Wöll, J. Am. Chem. Soc., 2007, 129, 15118.

39 C. Scherb, A. Schödel and T. Bein, Angew. Chem. Int. Ed., 2008, 47, 5777.

40 A. Schödel, C. Scherb and T. Bein, Angew. Chem. Int. Ed., 2010, 49, 7225.

12041 D. Zacher, K. Yusenko, A. Bétard, S. Henke, M. Molon, T. Ladnorg, O. Shekhah, B. Schüpbach, T. de los Arcos, M. Krasnopolski, M. Meilikhov, J. Winter, A. Terfort, C. Wöll and R. A. Fischer, Chem. Eur. J., 2011, 17, 1448.

42 O. Shekhah, H. Wang, D. Zacher, R. A. Fischer and C. Wöll, Angew. 125 Chem. Int. Ed., 2009, 48, 5038.

43 O. Shekhah, H. Wang, M. Paradinas, C. Ocal, B. Schüpbach, A. Terfort, D. Zacher, R. A. Fischer and C. Wöll, Nat. Mater., 2009, 8, 481. 
44 H. Wu, J. Yang, Z.-M. Su, S. R. Batten and J.-F. Ma, J. Am. Chem. Soc., 2011, 133, 11406.

45 H. K. Arslan, O. Shekhah, D. C. F. Wieland, M. Paulus, C. Sternemann, M. A. Schroer, S. Tiemeyer, M. Tolan, R. A. Fischer and C. Wöll, J. Am. Chem. Soc., 2011, 133, 8158.

46 S. Hermes, M. K. Schroter, R. Schmidt, L. Khodeir, M. Muhler, A. Tissler, R. W. Fischer and R. A. Fischer, Angew. Chem. Int. Ed., 2005, 44, 6237.

47 O. Shekhah, H. K. Arslan, K. Chen, M. Schmittel, R. Maul, W. $10 \quad$ Wenzel and C. Woll, Chem. Commun., 2011, 11210.

48 O. Zybaylo, O. Shekhah, H. Wang, M. Tafipolsky, R. Schmid, D. Johannsmann and C. Wöll, Phys. Chem. Chem. Phys., 2010, 12, 8092.

49 E. Biemmi, A. Darga, N. Stock and T. Bein, Micro. Meso. Mater., 2008, 114, 380.

50 P. Küsgens, M. Rose, I. Senkovska, H. Fröde, A. Henschel, S. Siegle, S. Kaskel, Micro. Meso. Mater., 2009, 120, 325.

51 H. Uehara, S. Diring, S. Furukawa, Z. Kalay, M. M. Tsotsalas, M. Nakahama, K. Hirai, M. Kondo, O. Sakata and S. Kitagawa, J. Am. Chem. Soc., 2011, 133, 11932.

52 K. C. Stylianou, R. Heck, S. Y. Chong, J. Bacsa, J. T. A. Jones, Y. Z. Khimyak, D. Bradshaw and M. J. Rosseinsky, J. Am. Chem. Soc., 2010, 132, 4119.

53 M. D. Allendorf, R. J. T. Houk, L. Andruszkiewicz, A. A. Talin, J. Pikarsky, A. Choudhury, K. A. Gall and P. A. Hesketh, J. Am. Chem. Soc., 2008, 130, 14404.

54 L. E. Kreno, J. T. Hupp and R. P. Van Duyne, Anal. Chem., 2010, 82, 8042.

55 K. Sugikawa, Y. Furukawa and K. Sada, Chem. Mater., 2011, 23, 3132.

56 J. An, S. J. Geib and N. L. Rosi, J. Am. Chem. Soc., 2009, 131, 8376.

57 A. M. Shultz, O. K. Farha, D. Adhikari, A. A. Sarjeant, J. T. Hupp and S. T. Nguyen, Inorg. Chem., 2011, 50, 3174.

58 D. Zacher, A. Baunemann, S. Hermes and R. A. Fischer, J. Mater. Chem., 2007, 17, 2785.

59 S. Hermes, D. Zacher, A. Baunemann, C. Wöll and R. A. Fischer, Chem. Mater., 2007, 19, 2168.

60 O. Kozachuk, K. Yusenko, H. Noei, Y. Wang, S. Walleck, T. Glaser and R. A. Fischer, Chem. Commun., 2011, 8509.

4061 J. Caro, M. Noack, P. Kölsch and R. Schäfer, Micro. Meso. Mater., 2000, 38, 3.

62 M. Arnold, P. Kortunov, D. J. Jones, Y. Nedellec, J. Karger and J. Caro, Eur. J. Inorg. Chem., 2007, 60.

63 J. Gascon, S. Aguado and F. Kapteijn, Micro. Meso. Mater. 2008, 113, 132.

64 S. Takamizawa, Y. Takasaki and R. Miyake, J. Am. Chem. Soc., 2010, 132, 2862.

65 R. Ranjan and M. Tsapatsis, Chem. Mater., 2009, 21, 4920.

66 Y. Hu, X. Dong, J. Nan, W. Jin, X. Ren, N. Xu and Y. M. Lee, Chem. Commun., 2011, 737.

67 H. Guo, G. Zhu, I. J. Hewitt and S. Qiu, J. Am. Chem. Soc., 2009, 131, 1646.

68 K. S. Park, Z. Ni, A. P. Cote, J. Y. Choi, R. Huang, F. J. UribeRomo, H. K. Chae, M. O'Keeffe and O. M. Yaghi, Proc. Nat. Acad. Sci., 2006, 103, 10186.

69 A. Phan, C. J. Doonan, F. J. Uribe-Romo, C. B. Knobler, M. O'Keeffe and O. M. Yaghi, Acc. Chem. Res., 2010, 43, 58.

70 K. Li, D. H. Olson, J. Seidel, T. J. Emge, H. Gong, H. Zeng and J. Li, J. Am. Chem. Soc., 2009, 131, 10368.

6071 H. Bux, F. Liang, Y. Li, J. Cravillon, M. Wiebcke and J. Caro, J. Am. Chem. Soc., 2009, 131, 16000.

72 A. Huang, H. Bux, F. Steinbach and J. Caro, Angew. Chem. Int. Ed., 2010, 49, 4958

73 Y.-S. Li, F.-Y. Liang, H. Bux, A. Feldhoff, W.-S. Yang and J. Caro, 65 Angew. Chem. Int. Ed., 2010, 49, 548

74 Y.-S. Li, H. Bux, A. Feldhoff, G.-L. Li, W.-S. Yang and J. Caro, Adv. Mater., 2010, 22, 3322.

75 H. Bux, A. Feldhoff, J. Cravillon, M. Wiebcke, Y.-S. Li and J. Caro, Chem. Mater., 2011, 23, 2262.

7076 D. Farien-Jimenez, S. A. Moggach, M. T. Wharmby, P. A. Wright, S. Parsons and T. Düren, J. Am. Chem. Soc., 2011, 133, 8900
77 S. Aguado, G. Bergeret, M. P. Titus, V. Moizan, C. Nieto-Draghi, N. Bats and D. Farrusseng, New. J. Chem., 2011, 35, 546.

78 W. Morris, C. J. Doonan, H. Furukawa, R. Banerjee and O. M. Yaghi, J. Am. Chem. Soc., 2008, 130, 12626.

79 A. Huang, W. Dou and J. Caro, J. Am. Chem. Soc., 2010, 132, 15562.

80 A. Huang and J. Caro, Angew. Chem. Int. Ed., 2011, 50, 4979.

81 S. Aguado, C.-H. Nicolas, V. Moizan-Basle, C. Nieto, H. Amrouche, N. Bats, N. Audebrand and D. Farrusseng, New. J. Chem., 2011, 35, 41.

82 S. R. Venna and M. A. Carreon, J. Am. Chem. Soc., 2010, 132, 76.

83 R. Makiura, S. Motoyama, Y. Umemura, H. Yamanaka, O. Sakata and H. Kitagawa, Nat. Mater., 2010, 9, 565.

84 S. Aguado, J. Canivet and D. Farrusseng, Chem. Comm., 2010, 7999.

8585 B. Chen, C. Liang, J. Yang, D. S. Contreras, Y. L. Clancy, E. B. Lobkovsky, O. M. Yaghi and S. Dai, Angew. Chem. Int. Ed., 2006, 45, 1390.

86 S. Han, Y. Wei, C. Valente, I. Lagzi, J. J. Gassensmith, A. Coskun, J. F. Stoddart and B. A. Grzybowski, J. Am. Chem. Soc., 2010, 132, 16358.

87 R. Ameloot, A. Liekens, L. Alaerts, M. Maes, A. Galarneau, B. Coq, G. Desmet, B. F. Sels, J. F. M. Denayer and D. E. de Vos, Eur. J. Inorg. Chem., 2010, 3735.

88 Z.-Y. Gu and X.-P. Yan, Angew. Chem. Int. Ed., 2010, 49, 1477.

9589 N. Chang, Z.-Y. Gu and X.-P. Yan, J. Am. Chem. Soc., 2010, 132, 13645.

90 S.-M. Xie, Z.-J. Zhang, Z.-Y. Wang and L.-M. Yuan, J. Am. Chem. Soc., 2011, 133, 11892.

91 D. Bradshaw, T. J. Prior, E. J. Cussen, J. B. Claridge and M. J. Rosseinsky, J. Am. Chem. Soc., 2004, 126, 6106.

92 A. S. Münch, J. Seidel, A. Obst, E. Weber and F. O. R. L. Mertens, Chem. Eur. J., 2011, 17, 10958.

93 A. M. B. Furtado, J. Liu, Y. Wang and M. D. LeVan, J. Mater. Chem., 2011, 21, 6698.

10594 S. B. Kim, C. Cai, S. Sun and D. A. Sweigart, Angew. Chem. Int. Ed., 2009, 48, 2907.

95 M. R. Lohe, K. Gedrich, T. Freudenberg, E. Kockrick, T. Dellmann and S. Kaskel, Chem. Commun., 2011, 3075.

96 F. Ke, Y.-P. Yuan, L.-G. Qiu, Y.-H. Shen, A.-J. Xie, J.-F. Zhu, X.-Y. Tian and L.-D. Zhang, J. Mater. Chem., 2011, 21, 3843.

97 R. Banerjee, Y. Katsenovich, L. Lagos, M. McIntosh, X. Zhang and C.-Z. Li, Curr. Med. Chem., 2010, 17, 3120.

98 P. Horcajada, T. Chalati, C. Serre, B. Gillet, C. Sebrie, T. Baati, J. F. Eubank, D. Heurtaux, P. Clayette, C. Kreuz, J.-S. Chang, Y. K. Hwang, V. Marsaud, P.-N. Bories, L. Cynober, S. Gil, G. Férey, P. Couvreur and R. Gref, Nat. Mater., 2010, 9, 172.

99 G. Lu, O. K. Farha, L. E. Kreno, P. M. Schoenecker, K. S. Walton, R. P. Van Duyne and J. T. Hupp, Adv. Mater., 2011, 23, 4449.

100 G. Lu and J. T. Hupp, J. Am. Chem. Soc., 2010, 132, 7832.

120101 Polymer synthesis: theory and practice; fundamentals, methods, experiments, D. Braun, H. Cherdron, M. Rehahn and H. Ritter, $4^{\text {th }}$ Edition, Springer, 2004.

102 C. de las Heras Alarcón, S. Pennadam and C. Alexander, Chem. Soc. Rev., 2005, 34, 276.

125103 M. G. Schwab, I. Senkovska, M. Rose, M. Koch, J. Pahnke, G. Jonschker and S. Kaskel, Adv. Eng. Mater., 2008, 10, 1151.

104 J. Yao, D. Dong, D. Li, L. He, G. Xu and H. Wang, Chem. Commun., 2011, 2559.

105 M. Meilikhov, K. Yusenko, E. Schollmeyer, C. Mayer, H.-J. Buschmann and R. A. Fischer, Dalton Trans., 2011, 40, 4838.

106 Y.-N. Wu, F. Li, Y. Xu, W. Zhu, C.-A. Tao, J. Cui and G. Li, Chem. Commun., 2011, 10094.

107 C. Liu, S. Kulprathipanja, A. M. W. Hillock, S. Husain and W. J. Koros, Adv. Membr. Tech Appl., 2008, 789.

135108 D. Q. Vu, W. J. Koros, S. J. Miller, J. Membr. Sci., 2003, 211, 311.

109 A. Centrone, Y. Yang, S. Speakman, L. Bromberg, G. C. Rutledge and T. A. Hatton, J. Am. Chem. Soc., 2010, 132, 15687.

110 Y. Zhang, I. H. Musselman, J. P. Ferraris and K. J. Balkus Jr., J. Membr. Sci., 2008, 313, 170.

140111 E. V. Perez, K. J. Balkus Jr., J. P. Ferraris and I. H. Musselman, J. Membr. Sci., 2009, 328, 165.

112 S. Keskin and D. S. Sholl, Energy Environ. Sci., 2010, 3, 343. 
113 T.-H. Bae, J. S. Lee, W. Qiu, W. J. Koros, C. W. Jones and S. Nair, Angew. Chem. Int. Ed., 2010, 49, 9863.

114 B. Zornoza, A. Martinez-Joaristi, P. Serra-Crespo, C. Tellez, J. Coronas, J. Gascon and F. Kapteijn, Chem. Commun., 2011, 9522.

5115 S. Basu, M. Maes, A. Cano-Odena, L. Alaerts, D. E. de Vos and I. F. J. Vankelecom, J. Membr. Sci., 2009, 344, 190.

116 X.-L. Liu, Y.-S. Li, G.-Q. Zhu, Y.-J. Ban, L.-Y. Xu and W.-S. Yang, Angew. Chem. Int. Ed., 2011, 50, 10639.

117 R. Ananthoji, J. F. Eubank, F. Nouar, H. Mouttaki, M. Eddaoudi and J. P. Harmon, J. Mater. Chem., 2011, 21, 9587.

118 R. Ostermann, J. Cravillon, C. Weidman, M. Wiebcke and B. M. Smarsly, Chem. Commun., 2011, 442

119 M. Rose, B. Böhringer, M. Jolly, R. Fischer and S. Kaskel, Adv. Eng. Mater., 2011, 13, 356.

15120 D. Li and Y. Xia, Adv. Mater., 2004, 16, 1151.

121 T. Chalati, P. Horcajada, R. Gref, P. Couvreur and C. Serre, J. Mater. Chem., 2011, 21, 2220.

122 C. Montoro, F. Linares, E. Q. Procopio, I. Senkovska, S. Kaskel, S. Galli, N. Masciocchi, E. Barea and J. A. R. Navarro, J. Am. Chem.

20 Soc., 2011, 133, 11888.

123 Carbon Nanotube Science, Peter J. F. Harris, Cambridge books 2009.

124 Carbon Nanotechnology, Ed. Liming Dai, Elsevier B.V. 2006.

125 C. Petit and T. J. Bandosz, Adv. Mater., 2009, 21, 4753.

126 C. Petit and T. J. Bandosz, Adv. Funct. Mater., 2010, 20, 111.

25127 C. Petit, B. Mendoza and T. J. Bandosz, Langmuir, 2010, 26, 15302.

128 C. Petit and T. J. Bandosz, Adv. Funct. Mater., 2011, 21, 2108.

129 C. Petit, J. Burress and T. J. Bandosz, Carbon, 2011, 49, 563.

130 C. Petit, B. Mendoza, D. O’Donnell and T. J. Bandosz, Langmuir, 2011, 27, 10234.

30131 C. Petit, B. Mendoza and T. J. Bandosz, ChemPhysChem, 2010, 11 3678.

132 M. Jahan, Q. Bao, J.-X. Yang and K. P. Loh, J. Am. Chem. Soc., 2010, 132, 14487.

133 S. J. Yang, J. Y. Choi, H. K. Chae, J. H. Cho, K. S. Nahm and C. R. Park, Chem. Mater. 2009, 21, 1893.

134 S. J. Yang, J. H. Cho, K. S. Nahm and C. R. Park, Int. J. Hydrogen Energy, 2010, 35, 13062.

135 X. Chen, P. Lukaszczuk, C. Tripisciano, M. H. Rümmeli, J. Srenscek-Nazzal, I. Pelech, R. J. Kalenczuk and E. Borowiak-Palen,

$40 \quad$ Phys. Status Solidi. B., 2010, 247, 2664.

136 Y. Li and R. T. Yang, J. Am. Chem. Soc., 2006, 128, 726.

137 K. P. Prasanth, P. Rallapalli, M. C. Raj, H. C. Bajaj and R. V. Jasra, Int. J. Hydrogen Energy, 2011, 36, 7594.

138 S. Yang, X. Lin, A. J. Blake, G. S. Walker, P. Hubberstey, N. R.

$45 \quad$ Champness and M. Schröder, Nat. Chem., 2009, 1, 487.

139 Z. Xiang, Z. Hu, D. Cao, W. Yang, J. Lu, B. Han and W. Wang, Angew. Chem. Int. Ed., 2011, 50, 491.

140 K. L. Mulfort, O. K. Farha, C. L. Stern, A. A. Sarjeant and J. T. Hupp, J. Am. Chem. Soc., 2009, 131, 3866.

50141 K. C. Stylianou, J. E. Warren, S. Y. Chong, J. Rabone, J. Bacsa, D. Bradshaw and M. J. Rosseinsky, Chem. Commun., 2011, 3389.

142 D. N. Dybtsev, A. L. Nuzhdin, H. Chun, K. P. Bryliakov, E. P. Talsi, V. P. Fedin and K. Kim, Angew. Chem. Int. Ed., 2006, 45, 916.

143 J. A. Rood, B. C. Noll and K. W. Henderson, J. Solid St. Chem., 2010, 183, 270.

144 I. Imaz, M. Rubio-Martínez, J. An, I. Solé-Font, N. L. Rosi and D. Maspoch, Chem. Commun., 2011, 7287.

145 J. M. Dominguez-Vera and E. Calacio, Inorg. Chem., 2003, 42, 6983.

146 J. Larionova, Y. Guari, C. Sangregorio and C. Guérin, New J. Chem., 2009, 33, 1177.

147 Y. Guari, J. Larionova, K. Molvinger, B. Folch and C. Guérin, Chem. Commun. 2006, 2613.

148 Y. Guari, J. Larionova, M. Corti, A. Lascialfari, M. Marinone, G. Poletti, K. Molvinger and C. Guérin, Dalton Trans., 2008, 3658.

65149 C. Clasen, T. Wilhelms and W.-M. Kulicke, Biomacromolecules, 2006, 7, 3210

150 W. Wei, L. Yuan, G. Hu, L.-Y. Wang, J. Wu, X. Hu, Z.-G. Su and G.-H. Ma, Adv. Mater., 2008, 20, 2292.

151 N. Kerbellec, L. Catala, C. Daiguebonne, A. Gloter, O. Stephan, J.-C. 70 Bünzli, O. Guillou and T. Mallah New J. Chem., 2008, 32, 584.
152 E. Chelebaeva, J. Larionova, Y. Guari, R. A. S. Ferreira, L. D. Carlos, A. A. Trifonov, T. Kalaivani, A. Lascialfari, C. Guérin, K. Molvinger, L. Datas, M. Maynadier, M. Gary-Bobo and M. Garcia, Nanoscale, 2011, 3, 1200.

75153 P. Küsgens, S. Siegle and S. Kaskel, Adv. Eng. Mater. 2009, 11, 93.

154 M. Uchida, M. T. Klem, M. Allen, P. Suci, M. Flenniken, E. Gillitzer, Z. Varpness, L. O. Liepold, M. Young and T. Douglas, Adv. Mater., 2007, 19, 1025.

155 P. M. Harrison and P. Arosio, Biochim. Biophys. Acta, 1996, 1275, 161.

156 C. C. Jolley, M. Uchida, C. Reichhardt, R. Harrington, S. Kang, M. T. Klem, J. B. Parise and T. Douglas, Chem. Mater., 2010, 22, 4612.

157 J. Lucon, M. J. Abedin, M. Uchida, L. Liepold, C. C. Jolley, M. Young and T. Douglas, Chem. Commun., 2010, 264.

85158 S. Jung, Y. Kim, S.-J. Kim, T. H. Kwon, S. Huh and S. Park, Chem. Commun., 2011, 2904.

159 C. Carbonell, I. Imaz and D. Maspoch, J. Am. Chem. Soc., 2011, 133, 2144.

160 Epitaxy: physical principles and technical implementation, M. A. $90 \quad$ Herman, W. Richter and H. Sitter, Springer., 2004.

161 K. Hirai, S. Furukawa, M. Kondo, H. Uehara, O. Sakata and S. Kitagawa, Angew. Chem. Int. Ed., 2011, 50, 8057.

162 S. Furukawa, K. Hirai, K. Nakagawa, Y. Takashima, R. Matsuda, T. Tsuruoka, M. Kondo, R. Haruki, D. Tanaka, H. Sakamoto, S. Shimomura, O. Sakata and S. Kitagawa, Angew. Chem. Int. Ed., 2009, 48, 1766.

163 K. Koh, A. G. Wong-Foy and A. J. Matzger, Chem. Commun., 2009, 6162.

164 S. Ferlay and W. Hosseini, Chem. Commun. 2004, 788.

100165 E. F. Bres, S. Ferlay, P. Dechambenoit, H. Leroux, M. W. Hosseini and S. Reyntjens, J. Mater. Chem., 2007, 17, 1559.

166 O. Shekhah, K. Hirai, H. Wang, H. Uehara, M. Kondo, S. Diring, D. Zacher, R. A. Fischer, O. Sakata, S.Kitagawa, S. Furukawa and C. Wöll, Dalton Trans., 2011, 40, 4954.

105167 Y. Yoo and H.-K. Jeong, Cryst. Growth Des., 2010, 10, 1283.

168 S. Furukawa, K. Hirai, Y. Takashima, K. Nagakawa, M. Kondo, T. Tsuruoka, O. Sakata and S. Kitagawa, Chem. Commun., 2009, 5097.

169 M. Kondo, S. Furukawa, K. Hirai and S. Kitagawa, Angew. Chem. Int. Ed., 2010, 49, 5327.

110170 Surfaces, Interfaces and Colloids, M. Drew, $2^{\text {nd }}$ Ed., Wiley-VCH, 1999.

171 Z. Wu and D. Zhao, Chem. Commun., 2011, 3332.

172 D. Zhao, Q. Huo, J. Feng, B. F. Chmelka and G. D. Stucky, J. Am. Chem. Soc., 1998, 120, 6024.

115173 F. Hoffmann, M. Cornelius, J. Morell and M. Fröba, Angew. Chem. Int. Ed., 2006, 45, 3216

174 X. Roy and M. J. MacLachlan, Chem. Eur. J., 2009, 15, 6552.

175 L.-G. Qiu, T. Xu, Z.-Q. Li, W. Wang, Y. Wu, X. Jiang, X.-Y. Tian and L.-D. Zhang, Angew. Chem. Int. Ed., 2008, 47, 9487.

120176 I. I. Slowing, B. G. Trewyn, S. Giri and V. S.-Y. Lin, Adv. Funct. Mater., 2007, 17, 1225.

177 Y. Zhao, J. Zhang, B. Han, J. Song, J. Li, and Q. Wang, Angew. Chem. Int. Ed., 2011, 50, 636.

178 J. Puigmarti-Luis, M. Rubio-Martinez, U. Hartfelder, I. Imaz, D. Maspoch and P. S. Dittrich, J. Am. Chem. Soc., 2011, 133, 4216.

179 R. Ameloot, F. Vermoortele, W. Vanhove, M. B. J. Roeffaers, B. F. Sels and D. E. De Vos, Nat. Chem., 2011, 3, 382.

180 M. Darbandi, H. K. Arslan, O. Shekhah, A. Bashir, A. Birkner and C. Wöll, Phys. Status Solidi RRL, 2010, 4, 197.

130181 Biomineralisation; principles and concepts in bioinorganic materials chemistry, Stephen Mann, Oxford Chemistry Masters series, OUP, 2001.

182 H. Cölfen and S. Mann, Angew. Chem. Int. Ed., 2003, 42, 2350.

183 S. Mann, Nat. Mater., 2009, 8, 781.

135 